\title{
Sigma non-opioid receptor 1 is a potential therapeutic target for long QT syndrome
}

\author{
LouJin Song, 1,2,12, Ramsey Bekdash ${ }^{11,2,12}$, Kumi Morikawa 1,12, Jose R. Quejada1,2,12, Alison D. Klein', \\ Danielle Aina-Badejo', Kazushige Yoshida1, Hannah E. Yamamoto1,3, Amy Chalan', Risako Yang ${ }^{1,4}$, \\ Achchhe Patel ${ }^{1,5}$, Dario Sirabella, ${ }^{1,5}$, Teresa M. Lee ${ }^{1,6}$, Leroy C. Joseph7, Fuun Kawano', \\ Junco S. Warren ${ }^{8,9,10}$, Rajesh K. Soni ${ }^{11}$, John P. Morrow ${ }^{7}$ and Masayuki Yazawa ${ }^{1,2} \bowtie$
}

\begin{abstract}
Some missense gain-of-function mutations in the CACNA1C gene, encoding calcium channel $\mathrm{Ca}_{\mathrm{v}} 1.2$, cause a life-threatening form of long QT syndrome (LQTS) named Timothy syndrome with currently no clinically effective therapeutics. Here we report that pharmacological targeting of sigma non-opioid intracellular receptor 1 (SIGMAR1) can restore electrophysiological function in induced pluripotent stem cell (iPSC)-derived cardiomyocytes generated from patients with Timothy syndrome and two common forms of LQTS, type 1 (LQTS1) and type 2 (LQTS2), caused by missense trafficking mutations in potassium channels. Electrophysiological recordings demonstrate that a Food and Drug Administration (FDA)-approved cough suppressant, dextromethorphan, can be used as an agonist of SIGMAR1 to shorten the prolonged action potential in cardiomyocytes from patients with Timothy syndrome and human cellular models of LQTS1 and LQTS2. When tested in vivo, dextromethorphan also normalized the prolonged QT intervals in a mouse model of Timothy syndrome. Overall, our study demonstrates that SIGMAR1 is a potential therapeutic target for Timothy syndrome and possibly other inherited arrhythmias such as LQTS1 and LQTS2.
\end{abstract}

alcium ions $\left(\mathrm{Ca}^{2+}\right)$ play an essential role in cardiac excitationcontraction coupling ${ }^{1,2}$. $\mathrm{Ca}^{2+}$-handling dysfunction is associated with various cardiac arrhythmias and genetic syndromes such as LQTS ${ }^{3,4}$. Some de novo gain-of-function mutations such as the one corresponding to G406R in cardiac L-type voltagegated $\mathrm{Ca}^{2+}$ channel $\mathrm{Ca}_{\mathrm{V}} 1.2$, which is encoded by CACNA1C, cause a lethal form of LQTS (type 8, also named Timothy syndrome $(\mathrm{TS}))^{3,5}$. Previously, using human patient-specific iPSCs, we found that the increased activity of cyclin-dependent kinase 5 (CDK5) is involved in the cardiac pathophysiological basis of TS (Fig. 1a, red) ${ }^{6}$. In addition, we observed that inhibiting CDK5 using genetic approaches and chemical inhibitors, such as PHA-793887, alleviated cellular phenotypes in cardiomyocytes derived from iPSCs of patients with $\mathrm{TS}^{6}$. However, currently available inhibitors of CDK5 also show inhibitory effects on other cyclin-dependent kinases. As a result, if the inhibitors are used for clinical application for treating cardiac diseases, it may lead to adverse events ${ }^{7}$ because cyclindependent kinases are known to play a critical role in cell cycle regulation. Moreover, developing CDK5-specific inhibitors is challenging due to the structural similarity of the catalytic domain in different cyclin-dependent kinases ${ }^{8}$. Therefore, using small molecules to directly target CDK5 is not ideal for further translational development to fulfill unmet medical needs of patients with TS. For this reason, we decided to search for alternative approaches that may modulate CDK5 activity in human cardiomyocytes. A comprehensive literature search returned SIGMAR1 as a potential target. SIGMAR1 is an emerging target for neurodegenerative diseases and cancer $^{9-11}$, playing an important role as a chaperone protein in endoplasmic reticulum membranes by regulating protein homeostasis, mitochondrial function, ion channel function and $\mathrm{Ca}^{2+}$ handling ${ }^{12}$. More importantly, activation of SIGMAR1 has been reported to suppress CDK5 activity in rodent neurons ${ }^{13}$, making SIGMAR1 activation an attractive alternative approach for CDK5 inhibition in TS models. Furthermore, the structure of SIGMAR1 has been uncovered ${ }^{14}$, which greatly facilitated the identification of SIGMAR1 ligands and understanding of interactions between SIGMAR1 and its ligands. Several SIGMAR1 agonists have been identified, including a potent and specific agonist, PRE-084 (ref. ${ }^{15}$ ), as well as several FDA-approved drugs such as fluvoxamine ${ }^{16}$ and dextromethorphan ${ }^{17-20}$ (Fig. 1a,b and Extended Data Fig. 1a). However, to our knowledge, little is known regarding the role of SIGMAR1 in cardiac ion channel regulation and pathophysiological conditions in cardiac arrhythmias. In this study, we examined the role of SIGMAR1 in the pathogenesis of LQTS as a therapeutic target and tested the hypothesis that treatment with a SIGMAR1 agonist, such as PRE-084, fluvoxamine or dextromethorphan, could rescue cardiac phenotypes in TS (Fig. 1a, blue).

\section{Results}

Effects of SIGMAR1 activation on cardiomyocytes from patients with TS. To examine whether SIGMAR1 activation modulates CDK5 activity in human cardiomyocytes as it did in neurons ${ }^{13}$, we conducted an in vitro luminescence-based CDK5 activity assay ${ }^{6}$ using cardiomyocytes derived from iPSCs of patients with TS and

'Columbia Stem Cell Initiative, Department of Rehabilitation and Regenerative Medicine, Columbia University, New York, NY, USA. ${ }^{2}$ Department of Pharmacology, Columbia University, New York, NY, USA. ${ }^{3}$ Barnard College, New York, NY, USA. ${ }^{4}$ Colgate University, Hamilton, NY, USA. ${ }^{5}$ Department of Genetics and Development, Columbia University, New York, NY, USA. ${ }^{6}$ Department of Pediatrics, Columbia University, New York, NY, USA. ${ }^{7}$ Department of Medicine, Columbia University, New York, NY, USA. ${ }^{8}$ Nora Eccles Harrison Cardiovascular Research and Training Institute, University of Utah, Salt Lake City, UT, USA. ${ }^{9}$ Department of Internal Medicine, University of Utah School of Medicine, Salt Lake City, UT, USA. ${ }^{\circ}$ Division of Developmental Genetics, Institute of Resource Developmental and Analysis, Kumamoto University, Kumamoto, Japan. "Proteomics and Macromolecular Crystallography Shared Resource, Columbia University, New York, NY, USA. ${ }^{12}$ These authors contributed equally: LouJin Song, Ramsey Bekdash, Kumi Morikawa, Jose R. Quejada. 凶e-mail: my2387@columbia.edu 


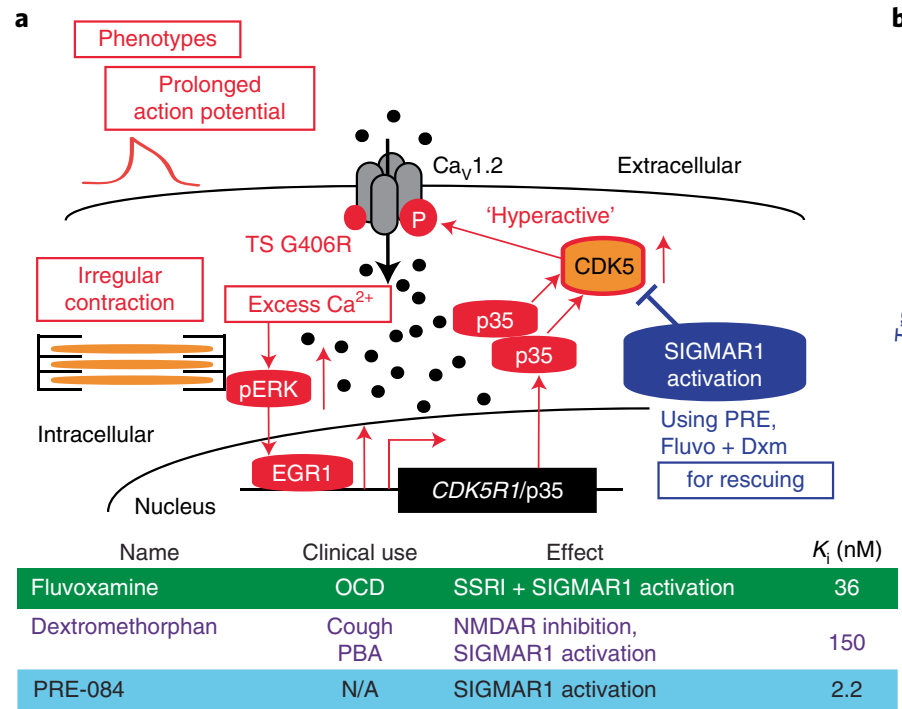

C

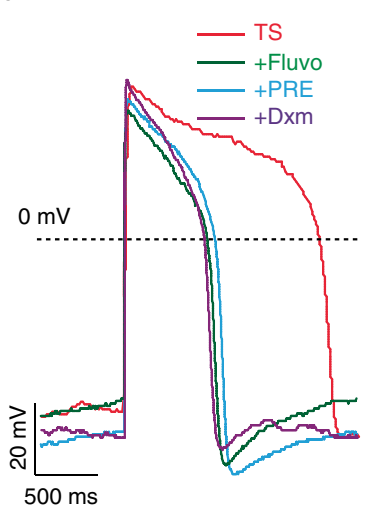

b

d

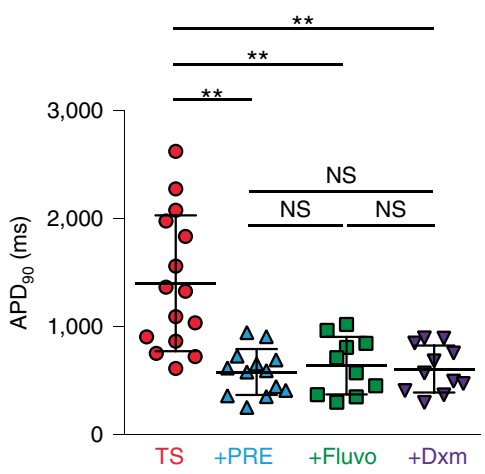

b SIGMAR1-PRE binding model
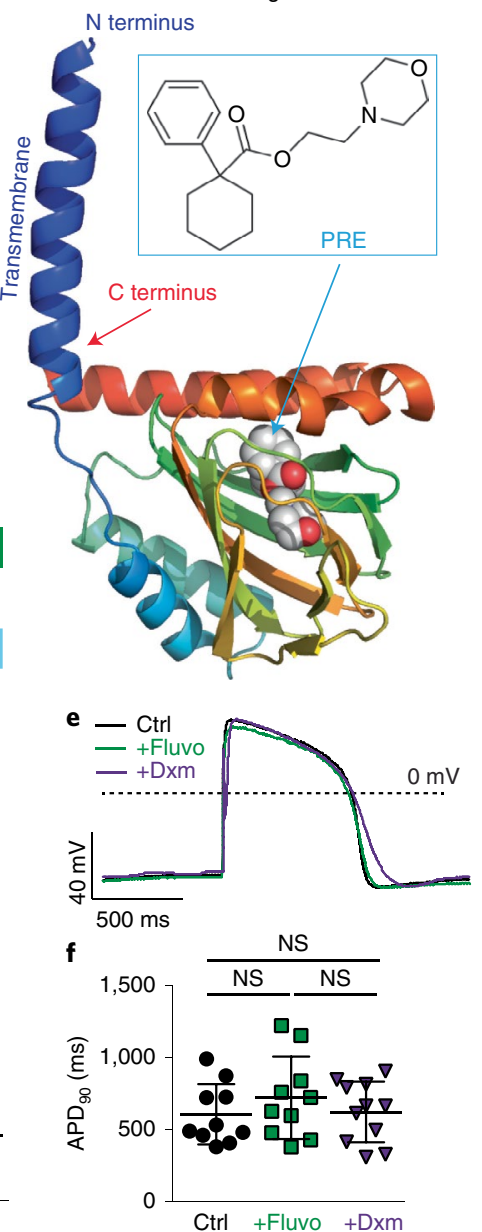

Fig. 1 | The effect of SIGMAR1 agonists on cardiac action potential phenotypes in cardiomyocytes derived from iPSCs of patients with TS. a, Schematic representation of altered molecules $(\text { red })^{6}$ and the therapeutic strategy using SIGMAR1 agonists (blue) in cardiomyocytes from patients with TS. The bottom table shows characteristics of SIGMAR1 agonists. EGR1, early growth response 1; pERK, phosphorylated extracellular signal-regulated kinase; OCD, obsessive compulsive disorder; PBA, pseudobulbar affect; N/A, not applicable; SSRI, selective serotonin reuptake inhibitor; NMDAR, N-methyl-Daspartate receptor; PRE, PRE-084; Fluvo, fluvoxamine; Dxm, dextromethorphan. b, Docking model of SIGMAR1 and its agonist PRE-084. c, Representative traces of $0.2-\mathrm{Hz}$-paced action potentials in cardiomyocytes from patients with TS without treatment or treated with SIGMAR1 agonists, PRE-084, fluvoxamine or dextromethorphan. d, $A_{P D_{90}}(90 \%$ reduction from peak) analysis of cardiomyocytes from patients with TS without treatment $(n=15)$ or treated with PRE-084 $(n=13)$, fluvoxamine $(n=10)$ or dextromethorphan $(n=11)$. Other action potential parameters are shown in Extended Data Fig. 2a-c. e, Representative traces of $0.5-\mathrm{Hz}$-paced action potentials in isogenic control (Ctrl) iPSC-derived cardiomyocytes without treatment or treated with dextromethorphan or fluvoxamine. $\mathbf{f}, \mathrm{APD}_{90}$ analysis of control cells without treatment $(n=10)$ or cells treated with dextromethorphan ( $\left.n=11\right)$ or fluvoxamine $(n=10)$. Other action potential parameters of isogenic control cells are shown in Extended Data Fig. $2 \mathrm{~d}-\mathrm{f}$. All data are mean \pm s.d. PRE-084, fluvoxamine and dextromethorphan treatment was carried out at $5 \mu \mathrm{M}$ for $2 \mathrm{~h}$. One-way ANOVA with Tukey's multiple comparisons was used for d,f. ${ }^{\star \star} P<0.01$; NS, not significant. Cardiomyocytes were from at least two independent differentiations.

treated them with the SIGMAR1-selective agonist PRE-084. Results indicated that treatment with PRE- 084 for $2 \mathrm{~h}$ significantly reduced CDK5 activity in cardiomyocytes from patients with TS (Extended Data Fig. 1b). While it was reported that protein expression of a CDK5-specific activator, $\mathrm{p} 35$ (CDK5R1), was regulated by SIGMAR1 in neurons ${ }^{13}$, interestingly, we observed no significant change in p35 protein expression between cardiomyocytes from patients with TS with and without PRE-084 treatment (Extended Data Fig. 1c,d). Instead, protein expression of CDK5 was significantly reduced in cardiomyocytes from patients with TS treated with PRE-084, while no significant changes in CDK5 transcript levels were detected (Extended Data Fig. 1c-e). These results revealed that activation of SIGMAR1 using its selective agonist PRE-084 could modulate CDK5 activity in cardiomyocytes from patients with TS. To further explore the molecular mechanism underlying reductions in CDK5 levels induced by PRE-084, we conducted a proximity ligation assay (PLA) to investigate the potential interaction between SIGMAR1 and CDK5. We found that SIGMAR1 directly interacted with CDK5 in cardiomyocytes from patients with TS and treatment with PRE-084 significantly increased the number of SIGMAR1-CDK5 PLA signals in cardiomyocytes from patients with TS, indicating an increased interaction between SIGMAR1 and CDK5 upon SIGMAR1 activation (Extended Data Fig. 1f,g). To validate that the change induced by PRE-084 was mediated by SIGMAR1, we treated cardiomyocytes from patients with TS with a potent and selective SIGMAR1 antagonist, NE-100 (refs. ${ }^{21-23}$ ), along with PRE-084, and used the cells in a SIGMAR1-CDK5 PLA. Results showed that cotreatment with NE-100 and PRE-084 reversed the effect of PRE084 on the interaction of SIGMAR1 with CDK5 in cardiomyocytes from patients with TS, confirming that the effect of PRE-084 was 
indeed mediated by SIGMAR1 (Extended Data Fig. 1f,g). Overall, results indicated that SIGMAR1 directly interacted with CDK5 in cardiomyocytes from patients with TS and SIGMAR1 activation led to an increased interaction between SIGMAR1 and CDK5 and reduced CDK5 expression and activity in cardiomyocytes from patients with TS.

The positive effect of SIGMAR1 activation on CDK5 activity prompted us to examine the effects of the SIGMAR1 agonist PRE-084 on cellular phenotypes of cardiomyocytes from patients with TS. We first examined the effect of PRE-084 on the irregular and slow contraction phenotype in cardiomyocytes from patients with TS using a contraction assay as reported previously ${ }^{6}$. This is because, as heart beats are normally regular, irregular contractions in a cellular model indicate electrophysiological instability. Imaging results demonstrated that PRE-084 alleviated irregular contraction and increased the beating rate of the patient cardiomyocytes (Extended Data Fig. 1h-j and Supplementary Videos 1 and 2). Next, we conducted whole-cell voltage-clamp recordings to evaluate the effect of PRE-084 on the phenotype in voltage-dependent inactivation of $\mathrm{Ca}_{\mathrm{V}} 1.2$ channels in cardiomyocytes from patients with TS. Results showed that PRE-084 treatment rescued the affected voltagedependent inactivation of $\mathrm{Ca}_{\mathrm{V}} 1.2$ in cardiomyocytes from patients with TS after treatment for $2 \mathrm{~h}$ but not after acute $(<10 \mathrm{~min})$ treatment (Extended Data Fig. 1k-n), supporting the beneficial effect of PRE-084 on cardiomyocytes from patients with TS. Furthermore, we examined the effect of PRE-084 on the prolonged action potential in cardiomyocytes from patients with TS and observed that PRE-084 significantly shortened the prolonged action potentials in patient cardiomyocytes (Fig. 1c,d and Extended Data Fig. 2a-c). Overall, these results demonstrated that the selective SIGMAR1 agonist PRE-084 ameliorated the phenotypes of cardiomyocytes from patients with TS. This points to SIGMAR1 activation as a potential therapeutic avenue for the cardiac phenotypes in TS.

Next, we examined the effects of fluvoxamine and dextromethorphan, which are also SIGMAR1 agonists, on the phenotypes of cardiomyocytes from patients with TS. The FDA-approved drugs fluvoxamine and dextromethorphan may have a greater translational potential for TS if the drugs are proved to be effective in a human cellular model. Similar to the selective SIGMAR1 agonist PRE-084, electrophysiological recordings revealed that the SIGMAR1 agonists fluvoxamine and dextromethorphan restored $\mathrm{Ca}_{\mathrm{V}} 1.2$ voltage-dependent inactivation and action potentials in cardiomyocytes from patients with TS (Fig. 1c,d and Extended Data Figs. 1k,l and 2a-c).

At the dose that fluvoxamine and dextromethorphan rescued the electrophysiological phenotypes in cardiomyocytes from patients with TS, the two FDA-approved drugs did not significantly alter the action potential duration (APD) of normal cardiomyocytes generated from isogenic control iPSC lines in which the mutation resulting in TS was previously corrected by gene-editing technology ${ }^{6}$ (Fig. 1e,f and Extended Data Fig. 2d-f). We sought to understand the difference in sensitivity to treatment with SIGMAR1 agonists between normal cardiomyocytes and those derived from patients with TS. We found that, at baseline, there was a significant increase in levels of SIGMAR1 transcripts and SIGMAR1 protein in cardiomyocytes from patients with TS compared with those in normal cardiomyocytes (Extended Data Fig. $2 g-j$ ), which may predispose cardiomyocytes from patients with TS to respond to SIGMAR1 agonists. To further explore the upstream pathway that regulates the expression of SIGMAR1, we examined the expression of ATF4, a transcription factor that has been reported to directly regulate SIGMAR1 transcription ${ }^{24}$. Results demonstrated that there was a significant increase in ATF4 protein levels (both non-phosphorylated and phosphorylated forms) and a significant decrease in ubiquitinated ATF4 protein levels in cardiomyocytes from patients with TS compared with those in their isogenic control cardiomyocytes
(Extended Data Fig. 2k-n), indicating an accumulation of ATF4 protein in cardiomyocytes from patients with TS. To confirm the regulatory role of ATF4 on SIGMAR1 expression in human cardiomyocytes, we overexpressed ATF4 in human normal cardiomyocytes and conducted quantitative PCR with reverse transcription (RT-qPCR) and western blotting to examine the effect of increased ATF4 expression on SIGMAR1 mRNA and SIGMAR1 protein expression. Results showed that ATF4 overexpression significantly increased the expression of SIGMAR1 transcripts and SIGMAR1 protein in cardiomyocytes derived from human iPSCs (Extended Data Fig. 2o-r), confirming the regulation of SIGMAR1 expression via ATF4. Altogether, results suggest that increased ATF4 protein levels in cardiomyocytes from patients with TS might activate the transcription of SIGMAR1 and upregulate the expression of SIGMAR1, leading to increased sensitivity of cardiomyocytes from patients with TS to treatment with SIGMAR1 agonists.

Although the observed beneficial effects of fluvoxamine and dextromethorphan on cardiomyocytes from patients with TS were both encouraging, dextromethorphan seems to hold greater therapeutic potential for translational application because of its wider age range for clinical use and favorable safety profile when used appropriately $y^{16,20,25-27}$. Thus, we decided to further characterize the effects of dextromethorphan. To examine the effect of dextromethorphan on the abnormal $\mathrm{Ca}^{2+}$-handling phenotype of cardiomyocytes from patients with TS, we used a genetically encoded fluorescent $\mathrm{Ca}^{2+}$ indicator, GCaMP6f ${ }^{28}$, for $\mathrm{Ca}^{2+}$ imaging. Compared with the use of $\mathrm{Ca}^{2+}$ dyes such as Fluo-4 AM, $\mathrm{Ca}^{2+}$ imaging using genetically encoded indicators has the distinct advantage that infected cells are intact and remain in reliable imaging condition for hours, which enables continuous monitoring of changes in $\mathrm{Ca}^{2+}$ handling after compound treatment for a longer time. Consistent with our previous findings using Fluo-4 AM for $\mathrm{Ca}^{2+}$ imaging ${ }^{5}, \mathrm{Ca}^{2+}$ imaging using GCaMP6f confirmed that cardiomyocytes from patients with TS demonstrated abnormal $\mathrm{Ca}^{2+}$ handling compared with isogenic control cardiomyocytes, and this phenotype could be stably monitored for at least $2 \mathrm{~h}$ after GCaMP6f fluorescent imaging began (Extended Data Fig. 3a-d). Using GCaMP6f-based $\mathrm{Ca}^{2+}$ imaging, we found that dextromethorphan treatment exhibited significant beneficial effects on the abnormal $\mathrm{Ca}^{2+}$-handling phenotype in cardiomyocytes from patients with TS and significantly increased $\mathrm{Ca}^{2+}$ transient frequency and shortened $\mathrm{Ca}^{2+}$ transient duration $2 \mathrm{~h}$ after treatment (Fig. $2 \mathrm{a}-\mathrm{c}$ ), which is also the time point at which we observed a significant beneficial effect of dextromethorphan on the electrophysiological phenotypes of cardiomyocytes from patients with TS (Fig. 1c,d).

Next, to further characterize the effect of dextromethorphan on L-type $\mathrm{Ca}^{2+}$ channel function in cardiomyocytes from patients with TS, we conducted whole-cell voltage-clamp recordings using $\mathrm{Ca}^{2+}$ as the charge carrier to examine the effect of dextromethorphan on $\mathrm{Ca}^{2+}$ channel currents. We found that dextromethorphan significantly reduced late $\mathrm{Ca}^{2+}$ currents in cardiomyocytes from patients with TS (Fig. 2d,e). This result, along with the result from electrophysiological recordings using $\mathrm{Ba}^{2+}$ as the charge carrier (Extended Data Figs. 11 and 3e), demonstrated that dextromethorphan treatment restored the inactivation of the $\mathrm{Ca}_{\mathrm{v}} 1.2$ channel in cardiomyocytes from patients with TS. In addition, to validate that the effect of dextromethorphan on $\mathrm{Ca}_{\mathrm{V}} 1.2$ channel inactivation was mediated by SIGMAR1, we tested the selective SIGMAR1 antagonist NE-100 (refs. ${ }^{21-23}$ ) along with dextromethorphan in electrophysiological recordings using $\mathrm{Ba}^{2+}$ as the charge carrier. We found that the beneficial effect of dextromethorphan on the affected voltage-dependent inactivation of $\mathrm{Ca}_{\mathrm{V}} 1.2$ in cardiomyocytes from patients with TS was reversed by cotreatment with NE-100, indicating that the beneficial effect of dextromethorphan on $\mathrm{Ca}^{2+}$ channel inactivation was mediated by SIGMAR1 (Fig. 2f,g). On the other hand, at the dose at which it was efficacious toward cardiomyocytes from patients with 
TS, dextromethorphan did not significantly alter voltage-dependent inactivation of $\mathrm{Ca}_{\mathrm{V}} 1.2$ in isogenic control cardiomyocytes (Extended Data Fig. 3f,g). Furthermore, as SIGMAR1 has been reported to interact with L-type $\mathrm{Ca}^{2+}$ channels and regulate channel function in retinal ganglion cells and dorsal ganglion neurons ${ }^{29-31}$, we examined the potential interaction between SIGMAR1 and $\mathrm{Ca}_{\mathrm{V}} 1.2$ in cardiomyocytes from patients with TS and the effect of dextromethorphan on the interaction using the PLA. Results suggest that SIGMAR1 directly interacted with $\mathrm{Ca}_{\mathrm{V}} 1.2$ in cardiomyocytes from patients with TS, and there was a significant reduction in the number of SIGMAR1-Ca 1.2 signals from the PLA in cardiomyocytes from patients with TS after dextromethorphan treatment (Extended Data Fig. 3h,i), indicating that dextromethorphan reduced the direct interaction between SIGMAR1 and $\mathrm{Ca}_{\mathrm{V}} 1.2$ in cardiomyocytes from patients with TS. Altogether, results demonstrated that dextromethorphan restored the channel inactivation of $\mathrm{Ca}_{\mathrm{V}} 1.2$ in cardiomyocytes from patients with TS through SIGMAR1 activation. The findings in aggregate indicated that SIGMAR1 might play a multifaceted role in regulating $\mathrm{Ca}_{\mathrm{V}} 1.2$ channel function in human iPSC-derived cardiomyocytes by not only regulating CDK5 activity but also by directly interacting with $\mathrm{Ca}_{\mathrm{v}} 1.2$.

To further investigate the effect of dextromethorphan on cardiac action potential, we performed voltage-clamp recordings to measure human Ether-à-go-go-Related Gene (hERG) currents in cardiomyocytes from patients with TS because potassium ion $\left(\mathrm{K}^{+}\right)$ channels are also known to play an important role in the repolarization phase of action potential in cardiomyocytes. Interestingly, we found that there was a significant reduction in $I_{\mathrm{Kr}}$ currents in cardiomyocytes from patients with TS compared with isogenic control cardiomyocytes (Extended Data Fig. 3j-1). Moreover, dextromethorphan treatment significantly increased $I_{\mathrm{Kr}}$ steady-state amplitude in cardiomyocytes from patients with TS, while no significant change in $I_{\mathrm{Kr}}$ currents was observed for isogenic control cardiomyocytes treated with dextromethorphan at the same dose (Fig. 2h,i and Extended Data Figs. 31 and $4 a-c$ ). Furthermore, the beneficial effect of dextromethorphan on $I_{\mathrm{Kr}}$ currents in cardiomyocytes from patients with TS was blocked by cotreatment with the SIGMAR1 antagonist NE-100 and dextromethorphan, confirming that the effect of dextromethorphan on hERG channels was mediated by SIGMAR1 (Fig. 2h,i and Extended Data Fig. 31,m). These results suggest that SIGMAR1 activation mediated by dextromethorphan might rescue prolonged action potentials in cardiomyocytes from patients with TS by restoring $\mathrm{K}^{+}$efflux in addition to reducing $\mathrm{Ca}^{2+}$ influx.

SIGMAR1 has been reported to directly interact with hERG channels in HEK cells ${ }^{32}$, and the direct interaction was further confirmed by atomic force microscopy ${ }^{33}$. In addition, SIGMAR1 silencing using short hairpin RNA or treatment with progesterone, which is also known to be a SIGMAR1 antagonist, decreased hERG current density and negatively regulated membrane expression of the hERG channel in leukemic cells, HEK cells and neonatal rat cardiomyocytes ${ }^{12,32,34}$, while SIGMAR1 overexpression potentiated hERG current density in Xenopus oocytes ${ }^{32}$. Thus, we decided to evaluate the potential interaction between SIGMAR1 and the hERG channel in cardiomyocytes derived from human iPSCs using the PLA and to examine the effect of dextromethorphan on their potential interaction. We found that, consistent with a previous report using HEK cells ${ }^{32}$, SIGMAR1 also directly interacted with hERG channels in cardiomyocytes derived from human iPSCs. Treatment with dextromethorphan for $2 \mathrm{~h}$ significantly reduced SIGMAR1-hERG signals in the PLA in cardiomyocytes from patients with TS, while no significant change in SIGMAR1-hERG signals in the PLA in isogenic control cardiomyocytes was observed with dextromethorphan treatment at the same dose (Fig. 2j,k and Extended Data Fig. 4d-g).

To investigate the molecular mechanisms underlying the effect of dextromethorphan on hERG channels further, we conducted immunocytochemistry for the hERG channel and plasma membrane with an anti-hERG antibody and the wheat germ agglutinin (WGA) fluorescent dye, respectively, using cardiomyocytes from patients with TS with and without treatment with dextromethorphan. We imaged the stained cardiomyocytes using a confocal microscope and then conducted blinded quantification for the colocalization of hERG and WGA fluorescent signals to examine the effect of dextromethorphan on the membrane localization of hERG in cardiomyocytes from patients with TS. The results suggested that there was a trend toward increased hERG-WGA colocalization in dextromethorphan-treated cardiomyocytes from patients with TS compared with that of untreated cells, but the change did not reach statistical significance (Extended Data Fig. 5a,b). In addition, we explored the possibility that SIGMAR1 activation induced by dextromethorphan treatment increased the expression of $\mathrm{KCNH} 2$ and hERG, resulting in the increased $I_{\mathrm{Kr}}$ observed in cardiomyocytes from patients with TS. Therefore, qPCR and western blotting

Fig. 2 The effect of dextromethorphan on cardiac $\mathrm{Ca}^{2+}$ handling and ion channels in a human iPSC model of TS. a, Representative traces of timecourse $\mathrm{Ca}^{2+}$ imaging in spontaneously contracting cardiomyocytes from patients with TS treated with dextromethorphan ( $5 \mu \mathrm{M}$, until 120 min). $\mathbf{b}, \mathbf{c}, \mathrm{Ca}^{2+}$ transient frequency $(\mathbf{b})$ and duration (c) analyses of cardiomyocytes from patients with TS before and after dextromethorphan treatment ( $\left.n=19\right)$. d, Representative traces of $\mathrm{Ca}^{2+}$ currents in cardiomyocytes from patients with TS with and without dextromethorphan. $\mathbf{e}$, Late $\mathrm{Ca}{ }^{2+}$ current analysis of cardiomyocytes from patients with TS with and without dextromethorphan treatment (TS, $n=11$; with dextromethorphan, $n=12)$. f, Representative traces of $\mathrm{Ba}^{2+}$ currents in cardiomyocytes from patients with TS without treatment or treated with dextromethorphan $(5 \mu \mathrm{M}, 2 \mathrm{~h}$, dextromethorphan) or with dextromethorphan and a SIGMAR1 antagonist, NE-100 (1 $\mu \mathrm{M}$, dextromethorphan and NE-100). g, Voltage-dependent inactivation in cardiomyocytes from patients with TS without treatment $(n=25)$ or treated with dextromethorphan $(n=16)$ or with dextromethorphan and NE-100 $(n=11)$. h, Representative traces of $I_{\mathrm{Kr}}$ currents (E-4031 sensitive) in cardiomyocytes from patients with TS treated with dextromethorphan $(5 \mu \mathrm{M})$ or dextromethorphan and $\mathrm{NE}-100$ (each at $5 \mu \mathrm{M}$ ) or without treatment. $\mathbf{i}, I_{\mathrm{Kr}}$ current amplitude analysis of cardiomyocytes from patients with TS treated with dextromethorphan $(n=9)$ or dextromethorphan and NE-100 $(n=10)$ or without treatment $(n=10)\left({ }^{\star} P<0.05\right.$, dextromethorphan versus TS and dextromethorphan versus dextromethorphan and NE-100 at $-10,0,10,20$ and $30 \mathrm{mV}$ ). j, Representative confocal fluorescent and bright-field images of cardiomyocytes from patients with TS without and with dextromethorphan treatment from the PLA (SIGMAR1-hERG, red; 4,6-diamidino-2-phenylindole (DAPI), blue). Scale bar, $10 \mu \mathrm{m}$. k, Quantification of SIGMAR1-hERG by the PLA in dextromethorphan-treated $(n=36)$ and untreated $(n=35)$ cardiomyocytes from patients with TS. I, Representative traces of $I_{\mathrm{KS}}$ currents (chromanol 293B sensitive) in cardiomyocytes from patients with TS treated with dextromethorphan $(5 \mu \mathrm{M})$ or dextromethorphan and NE-100 (each at $5 \mu \mathrm{M}$ ) or without treatment. $\mathbf{m}, I_{\mathrm{Ks}}$ current amplitude analysis of cardiomyocytes from patients with TS treated with dextromethorphan $(n=10)$ or dextromethorphan and NE-100 $(n=9)$ or without treatment $(n=10)\left({ }^{*} P<0.05\right.$, dextromethorphan versus TS and dextromethorphan versus dextromethorphan and NE-100 at $-10,0,10,20,30$ and $40 \mathrm{mV}$ ). $\mathbf{n}$, Representative epifluorescent and phase-contrast images of cardiomyocytes from patients with TS without and with dextromethorphan treatment from the PLA (SIGMAR1-K 7.1 , red; DAPI, blue). Scale bar, $10 \mu \mathrm{m}$. $\mathbf{0}$, Quantification of SIGMAR1-K 7.1 by the PLA in dextromethorphan-treated $(n=40)$ and untreated $(n=20)$ cardiomyocytes from patients with TS. All data are mean \pm s.d. One-way ANOVA with Tukey's multiple comparisons was used for $\mathbf{b}, \mathbf{c}, \mathbf{g}$ between groups and for $\mathbf{i}, \mathbf{m}$ at each voltage step. Unpaired two-tailed Student's $t$-tests were used for $\mathbf{e}, \mathbf{k}, \mathbf{0} .{ }^{\star} P<0.05,{ }^{\star \star} P<0.01,{ }^{\star \star \star} P<0.001$. Samples were from at least two independent differentiations. 
were conducted to examine the expression of $\mathrm{KCNH} 2$ transcripts and hERG protein in cardiomyocytes from patients with TS with or without dextromethorphan treatment. We found that dextromethorphan significantly increased $\mathrm{KCNH} 2$ transcription in patient cardiomyocytes (Extended Data Fig. 5c) and did not significantly alter the protein expression of hERG (Extended Data Fig. 5d,e).

Overall, these results demonstrated that SIGMAR1 directly interacted with hERG channels in human iPSC-derived cardiomyocytes, and the SIGMAR1 agonist dextromethorphan might increase $I_{\mathrm{Kr}}$ currents by dissociating the interaction between SIGMAR1 and hERG channels in cardiomyocytes from patients with TS, while the exact mechanisms remain to be characterized.

The observation of an interaction between SIGMAR1 and the hERG channel in human iPSC-derived cardiomyocytes prompted us to examine whether there is a similar interaction between SIGMAR1 and another $\mathrm{K}^{+}$channel, $\mathrm{K}_{\mathrm{V}}$ 7.1, which is encoded by the
KCNQ1 gene and also plays an important role in the repolarization phase of cardiac action potentials, similar to the hERG channel. Using electrophysiological recordings, we found that, similar to our observations of the hERG channel, cardiomyocytes from patients with TS showed significantly reduced $I_{\mathrm{Ks}}$ currents compared with isogenic controls and treatment with dextromethorphan significantly increased $I_{\mathrm{Ks}}$ currents in cardiomyocytes from patients with TS (Fig. 2l,m and Extended Data Fig. 5f,g). In addition, cotreatment with NE-100 and dextromethorphan blocked the beneficial effect of dextromethorphan on $I_{\mathrm{Ks}}$ currents in cardiomyocytes from patients with TS, confirming that the effect of dextromethorphan on $\mathrm{K}_{\mathrm{V}} 7.1$ channels is mediated by SIGMAR1 (Fig. 2l,m and Extended Data Fig. 5g). Moreover, consistent with our observations for SIGMAR1 and hERG channels, SIGMAR1 also directly interacted with the $\mathrm{K}_{\mathrm{V}} 7.1$ channel in human iPSC-derived cardiomyocytes. Similarly, dextromethorphan treatment significantly reduced SIGMAR1-K 7.1 a
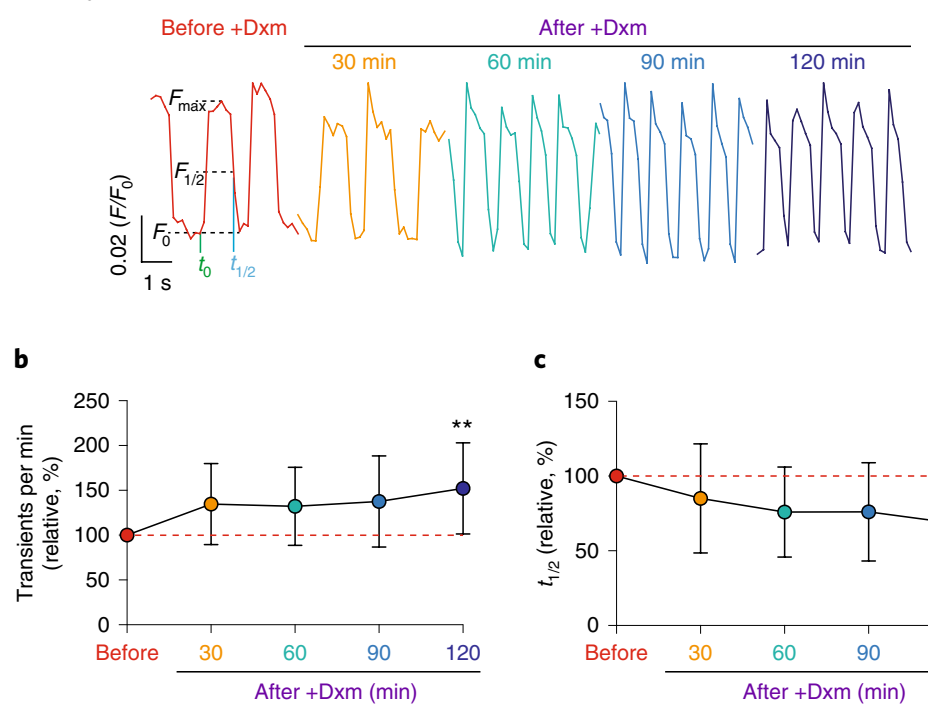

h
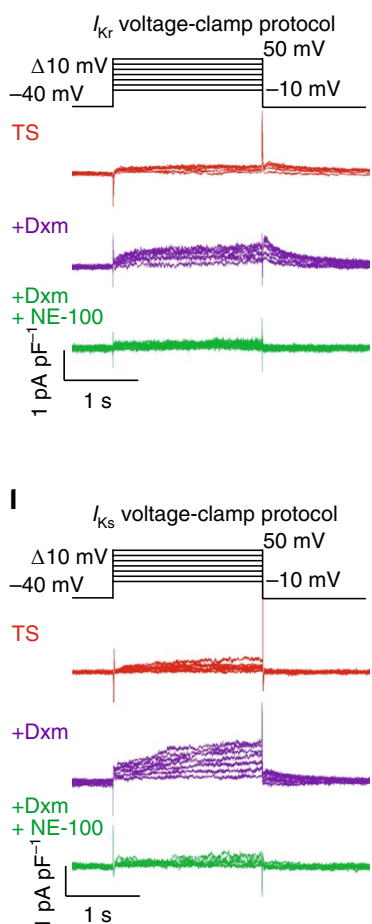

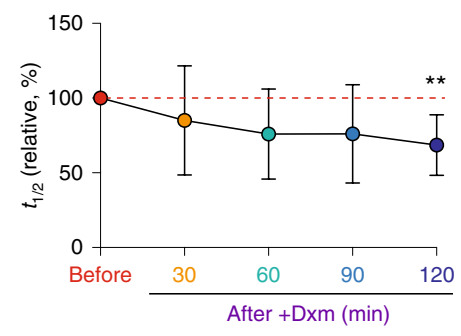

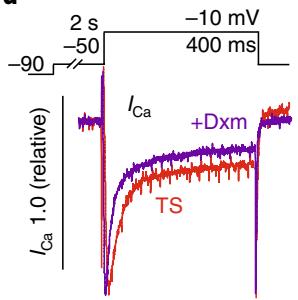

f
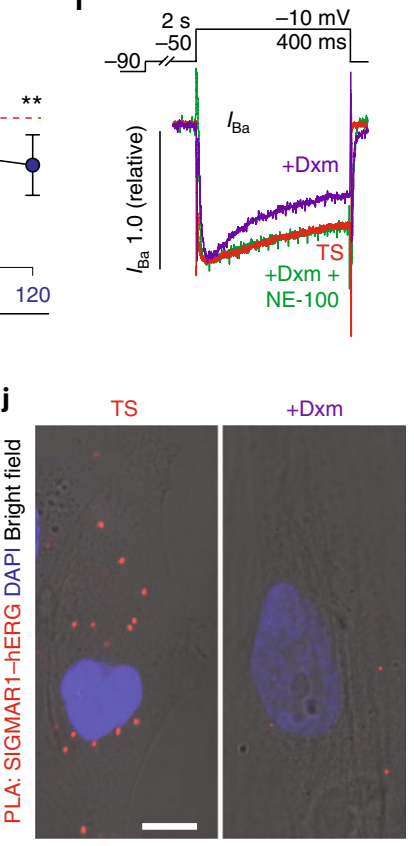

(Red, confocal fluorescence)

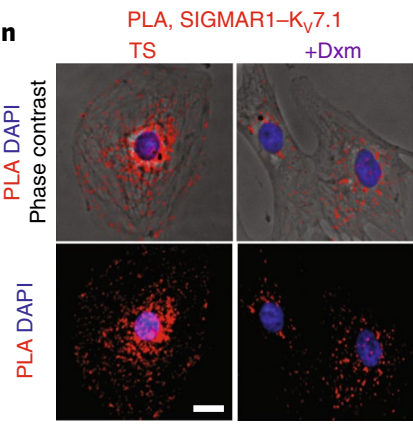

(Red, epifluorescence)
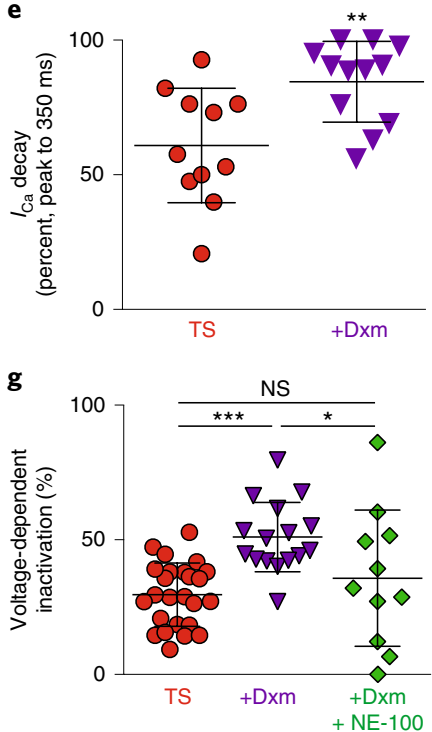

k
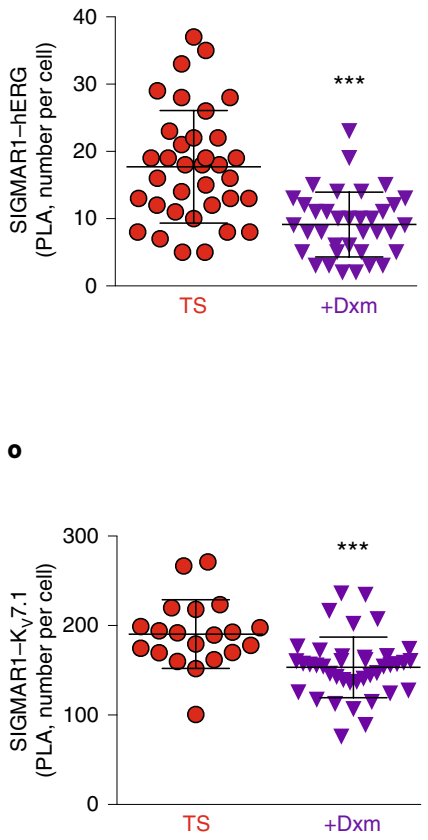
signals in the PLA in cardiomyocytes from patients with TS (Fig. 2n,o). On the other hand, there were no significant changes in $I_{\mathrm{Ks}}$ currents in isogenic control cardiomyocytes after dextromethorphan treatment at the same dose, although a reduction in SIGMAR1-K $\mathrm{K}_{\mathrm{V}} 7.1$ signals in the PLA was observed upon treatment (Extended Data Fig. 4h-k).

To investigate the molecular mechanisms underlying the effect of dextromethorphan on the $\mathrm{K}_{\mathrm{V}} 7.1$ channel further, we conducted immunocytochemistry for the $\mathrm{K}_{\mathrm{V}} 7.1$ channel (encoded by KCNQ1) and plasma membrane using an anti- $\mathrm{K}_{\mathrm{V}} 7.1$ antibody and the WGA fluorescent dye, respectively, and cardiomyocytes from patients with TS with and without dextromethorphan treatment. A significant increase in plasma membrane localization of the $\mathrm{K}_{\mathrm{V}} 7.1$ channel was observed upon dextromethorphan treatment (Extended Data Fig. 5h,i), indicating that SIGMAR1 activation induced by dextromethorphan treatment facilitated membrane localization of $\mathrm{K}_{\mathrm{V}} 7.1$ channels in cardiomyocytes from patients with TS. In addition, we conducted qPCR to examine the expression of KCNQ1 transcripts in cardiomyocytes from patients with TS with and without dextromethorphan treatment and observed a significant increase in the transcription of KCNQ1 isoform 1 upon dextromethorphan treatment, while transcription of KCNQ1 isoform 2 was not significantly altered by dextromethorphan treatment (Extended Data Fig. 5j). Consistent with the qPCR results, western blotting showed a significant increase in the expression of the $K_{V} 7.1$ isoform 1 protein but not in that of the isoform 2 protein upon dextromethorphan treatment (Extended Data Fig. 5k-m), indicating that SIGMAR1 activation induced by dextromethorphan treatment increased the expression of $\mathrm{K}_{\mathrm{V}} 7.1$ isoform 1 in addition to facilitating membrane localization of $\mathrm{K}_{\mathrm{V}} 7.1$ channels.

In summary, these results demonstrated that, in addition to the hERG channel, SIGMAR1 also directly interacted with the $\mathrm{K}_{\mathrm{V}} 7.1$ channel in human iPSC-derived cardiomyocytes, and the SIGMAR1 agonist dextromethorphan might increase $I_{\mathrm{Ks}}$ currents in cardiomyocytes from patients with TS by dissociating the interaction between SIGMAR1 and $\mathrm{K}_{\mathrm{V}} 7.1$ channels, increasing plasma membrane localization of $\mathrm{K}_{\mathrm{V}} 7.1$ and increasing expression of $\mathrm{K}_{\mathrm{V}} 7.1$ isoform 1 .

Effects of SIGMAR1 activation on cardiomyocytes from patients with LQTS1 and LQTS2. The observation that dextromethorphan increased $I_{\mathrm{Kr}}$ and $I_{\mathrm{Ks}}$ currents in cardiomyocytes from patients with TS motivated us to examine the effect of dextromethorphan on cardiomyocytes derived from human iPSCs of two common types of LQTS, LQTS1 and LQTS2, carrying missense trafficking mutations corresponding to G269S in the $\mathrm{K}_{\mathrm{V}} 7.1$ channel, and A561V in the hERG channel, respectively. The missense trafficking mutations in the $\mathrm{K}_{\mathrm{V}} 7.1$ and hERG channels were previously reported to decrease $\mathrm{K}^{+}$currents and prolong action potentials in human iPSC-derived cardiomyocytes ${ }^{35,36}$. First, we used CRISPR-Cas9 gene-editing technology to correct the mutations in our iPSCs derived from patients with LQTS1 or LQTS2 and generated isogenic control iPSCs (Extended Data Fig. 6a-d). Next, we differentiated iPSCs from both patients with LQTS1 and LQTS2 along with their corresponding isogenic controls into cardiomyocytes and evaluated their electrophysiological phenotypes. Consistent with previous reports ${ }^{35,36}$, we found that cardiomyocytes derived from iPSCs of patients with LQTS1 or LQTS2 showed prolonged action potentials (APD, 90\% from peak $\left(\mathrm{APD}_{90}\right)$, mean \pm s.d., LQTS1 versus the isogenic control, $904.3 \pm 337.0 \mathrm{~ms}$ versus $522.9 \pm 186.6 \mathrm{~ms}, P=0.004$; LQTS2 versus the isogenic control, $1,054.2 \pm 398.9 \mathrm{~ms}$ versus $644.8 \pm 201.9$ ms, $P=0.011$; unpaired two-tailed Student's $t$-test) and also that cardiomyocytes from patients with LQTS1 or LQTS2 showed significantly reduced $\mathrm{K}^{+}$currents in electrophysiological recordings compared with their corresponding isogenic control cardiomyocytes (Extended Data Fig. 6j,k,q-s). Treatment with a SIGMAR1 agonist, fluvoxamine or dextromethorphan, significantly shortened the prolonged action potential in cardiomyocytes from patients with LQTS1 or LQTS2 (Figs. 3a,b and 4a,b and Extended Data Fig. 7a-f). In addition, consistent with the effects of dextromethorphan on $\mathrm{K}^{+}$channels in cardiomyocytes from patients with TS, dextromethorphan treatment significantly increased both $I_{\mathrm{Kr}}$ and $I_{\mathrm{Ks}}$ currents in cardiomyocytes from patients with LQTS1 or LQTS2 (Figs. $3 \mathrm{c}-\mathrm{f}$ and $4 \mathrm{c}-\mathrm{f}$ and Extended Data Fig. $7 \mathrm{~g}, \mathrm{~h}$ ). Mechanistically, similar to what was observed in cardiomyocytes from patients with TS (Fig. $2 \mathrm{j}, \mathrm{k}, \mathrm{n}, \mathrm{o}$ ), dextromethorphan treatment significantly reduced both SIGMAR1-hERG and SIGMAR1-K 7.1 signals in the PLA in cardiomyocytes from patients with LQTS1 or LQTS2 (Figs. $3 g-j$ and $4 \mathrm{~g}-\mathrm{j}$ ). Moreover, at the efficacious dose, dextromethorphan did not exhibit significant effects on action potentials in the corresponding isogenic control cardiomyocytes (Extended Data Fig. 6e-i,l-p) or on the voltage-dependent inactivation of $\mathrm{Ca}_{\mathrm{V}} 1.2$ in cardiomyocytes from patients with LQTS1 or LQTS2 (Extended Data Fig. 7i-n). Furthermore, a significant increase in SIGMAR1 and ATF4 expression was detected in cardiomyocytes from patients with LQTS1 or LQTS2, which may potentiate the cardiomyocytes to respond to dextromethorphan treatment, while no significant changes in CDK5 or p35 expression were observed in cardiomyocytes from patients with LQTS1 or LQTS2 compared with control cardiomyocytes (Extended Data Fig. 7o-w). Overall, these results demonstrated that the SIGMAR1 agonist dextromethorphan increased $\mathrm{K}^{+}$ currents in cardiomyocytes from patients with LQTS1 or LQTS2 by activating SIGMAR1 and that dextromethorphan treatment could be beneficial for LQTS1 and LQTS2 (caused by trafficking mutations), which are more common forms of LQTS than TS.

Effects of dextromethorphan on a mouse model of TS. Based on the intriguing in vitro results, we decided to conduct in vivo studies to examine the effect of dextromethorphan on TS further. We generated a cardiac-specific mouse model of TS, taking advantage of an inducible expression system using the Rosa26 locus, a cardiac Cre driver ( $\alpha$-MHC/Myf $\left.6^{\text {MerCreMer }}\right)$ and a loxP pair with stop cassette to conditionally overexpress mutant channels from TS in the heart (Fig. 5a $)^{37}$. The inducible expression system allows us to express mutant channels at the adult stage to avoid heart developmental phenotypes that may lead to early lethality. A common challenge in the field of LQTS concerning murine models is the failure to recapitulate arrhythmic phenotypes in vivo ${ }^{38}$ due to the fact that mice have different ion channel expression profiles and a remarkably higher heart rate than humans. Fortunately, our cardiac-specific mouse model of TS successfully recapitulated the LQTS phenotype and showed prolongation of the QT interval at $4 \mathrm{~d}$ after tamoxifen administration as revealed by electrocardiography (ECG) (Fig. 5b-d). Next, we examined the effect of dextromethorphan on the LQTS phenotype by adding dextromethorphan to drinking water for dosing. A low dose of quinidine was also added with dextromethorphan to improve the pharmacokinetic profile of dextromethorphan in vivo, as quinidine is known to competitively inhibit the metabolism of dextromethorphan to dextrorphan. Clinically, a low dose of quinidine together with dextromethorphan is used to maintain a stable increase in the plasma concentration of dextromethorphan in humans ${ }^{19,20}$. To examine the pharmacokinetic profile of dextromethorphan with the dosing strategy, blood was sampled from mice at different time points on days 4 and 11 to measure dextromethorphan concentrations in plasma. The results indicated that the dosing strategy for dextromethorphan used in the study achieved a stable increase in plasma dextromethorphan levels throughout the day (Extended Data Fig. 8a), with the plasma level of dextromethorphan in treated animals being comparable to dextromethorphan levels in human participants taking $30 \mathrm{mg}$ dextromethorphan per day for $7 \mathrm{~d}\left(\sim 10-60 \mathrm{ng} \mathrm{ml}^{-1}\right)^{19}$. Next, we examined the effect of dextromethorphan on the LQTS phenotype in the mouse model of TS 


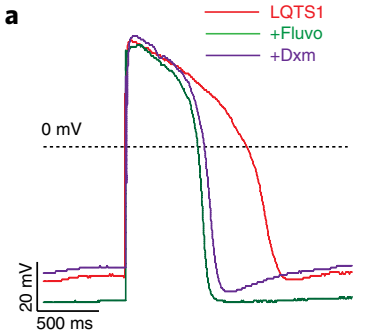

b
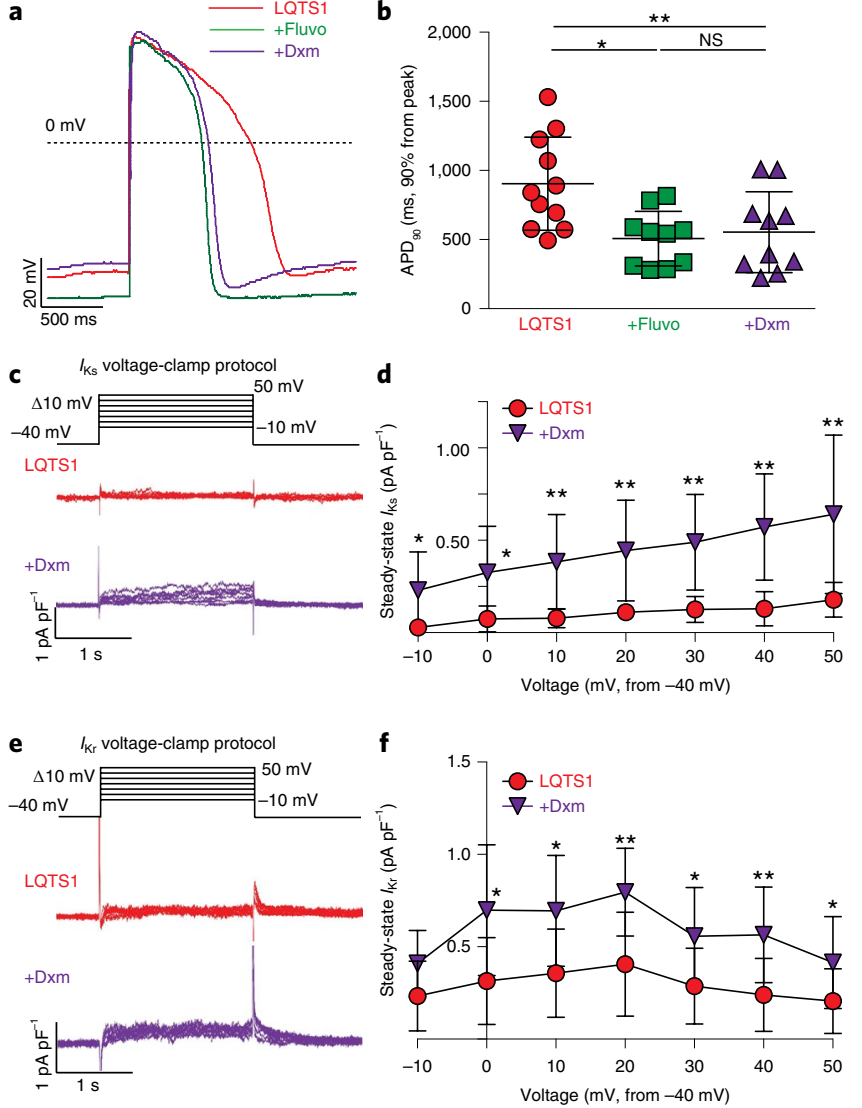
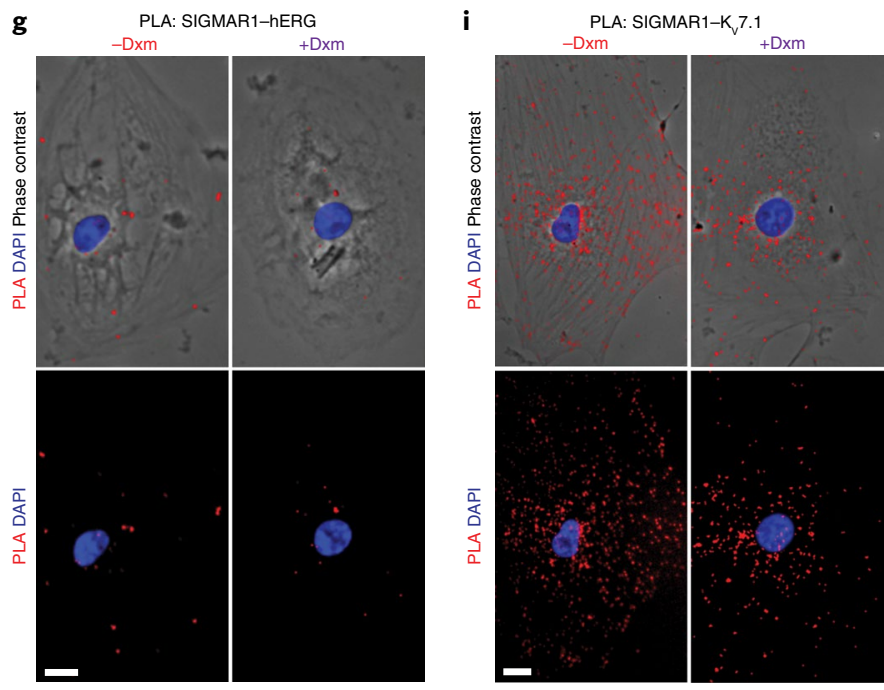

h

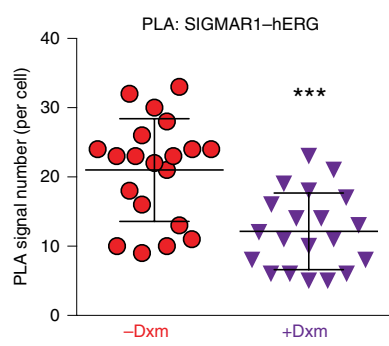

j

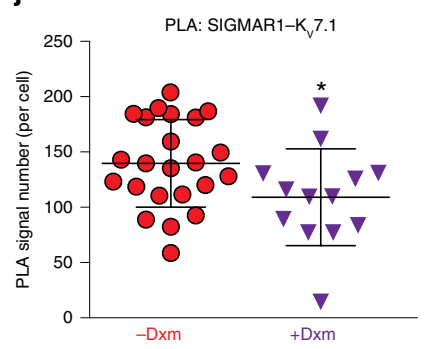

Fig. 3 | The effect of dextromethorphan on phenotypes in LQTS1. a, Representative traces of action potentials in cardiomyocytes derived from iPSCs of patients with LQTS1 without treatment or treated with fluvoxamine or dextromethorphan. $\mathbf{b}, \mathrm{APD}_{90}$ analysis of cardiomyocytes from patients with LQTS1 without treatment $(n=11)$ or treated with fluvoxamine $(n=10)$ or dextromethorphan $(n=10)$. Other action potential parameters are shown in Extended Data Fig. 7a,c,e. c, Representative traces of $I_{\mathrm{Ks}}$ currents (chromanol 293B sensitive) in cardiomyocytes from patients with LQTS1 treated with dextromethorphan or untreated. $\mathbf{d}, I_{\mathrm{Ks}}$ currents (steady state) were significantly increased by dextromethorphan treatment of cardiomyocytes from patients with LQTS1 at all voltage steps ( $n=10$ per group). e, Representative traces of $I_{\mathrm{kr}}$ currents (E-4031 sensitive) in cardiomyocytes from patients with LQTS1 treated with dextromethorphan or untreated. $\mathbf{f}, I_{\mathrm{kr}}$ currents (steady state) were significantly increased in dextromethorphan-treated cardiomyocytes from patients with LQTS1 $(n=9)$ at 0-, 10-, 20-, 30-, 40- and 50-mV steps compared with those of cardiomyocytes from patients with LQTS1 without treatment $(n=10)$. g, Representative epifluorescent and phase-contrast images of cardiomyocytes from patients with LQTS1 with and without dextromethorphan treatment from the PLA (SIGMAR1-hERG, red; DAPI, blue). Scale bar, $10 \mu \mathrm{m}$. h, Quantification of SIGMAR1-hERG by the PLA in dextromethorphan-treated $(n=20)$ and untreated $(n=20)$ cardiomyocytes from patients with LQTS1. $\mathbf{i}$, Representative epifluorescent and phase-contrast images of cardiomyocytes from patients with LQTS1 with and without dextromethorphan treatment from the PLA (SIGMAR1-K 7.1 , red; DAPI, blue). Scale bar, $10 \mu \mathrm{m}$. j, Quantification of SIGMAR1-K 7.1 by the PLA in dextromethorphan-treated $(n=13)$ and untreated $(n=23)$ cardiomyocytes from patients with LQTS1. All data are mean \pm s.d. Treatment with fluvoxamine or dextromethorphan was carried out at $5 \mu \mathrm{M}$ for $2 \mathrm{~h}$. One-way ANOVA with Tukey's multiple comparisons was used for $\mathbf{b}$, and unpaired two-tailed Student's $t$-tests were used for $\mathbf{h}, \mathbf{j}$ between groups and for $\mathbf{d}$, $\mathbf{f}$ at each voltage step. ${ }^{\star} P<0.05$, ${ }^{\star \star} P<0.01,{ }^{\star \star \star} P<0.001$. Cardiomyocytes were from at least two independent differentiations.

using ECG at day 4 and day 11. Dextromethorphan-quinidine significantly shortened the prolonged QT interval in the mouse model of TS at both time points (Fig. $5 c, d$ and Extended Data Fig. 8b,c). Moreover, dextromethorphan-quinidine did not significantly affect QT intervals in control littermate mice (Fig. 5c,d), which is consistent with clinical reports indicating no alteration of QT intervals in human participants when dextromethorphan-quinidine was used to treat pseudobulbar affect ${ }^{19,20}$. The overall results demonstrated that dextromethorphan not only rescued the phenotypes in cardiomyocytes derived from iPSCs of patients with TS in vitro but also alleviated the LQTS phenotype in the mouse model of TS in vivo.

Lastly, to profile global changes in hearts of the mouse model of TS and the effects of dextromethorphan on mutant hearts, we performed a global proteomic analysis to profile protein expression changes in the hearts of controls, untreated and dextromethorphantreated mice in the TS model at day 11. Proteomic results demonstrated that expression of many important molecules involved in cardiac excitation-contraction coupling, mitochondrial function, heart failure, fibrosis and cardiomyopathy (such as the TRIC channel (Tmem38a), atrial natriuretic peptide (ANP) (Nppa), periostin (Postn) and cardiomyopathy-associated gene 3 (CMYA3, Xirp2) ${ }^{39-42}$ ) was altered in mouse hearts in the TS model, while dextromethorphan treatment restored expression of these molecules to a level comparable to that of controls (Fig. 5e and Extended Data Fig. 9). This supports the efficacy of dextromethorphan on hearts in the TS model at the endpoint, even though dextromethorphan treatment did not restore the expression of all molecules involved in pathways related to cardiac dysfunction and arrhythmias. In addition, interestingly, the proteomic analysis indicated that dextromethorphan treatment induced a significant increase in the expression of the $\mathrm{K}_{\mathrm{V}} 7.1$ (Kcnq1) protein in hearts in the TS model (Fig. 5e and Extended Data Fig. 9), which is consistent with our observation using cardiomyocytes derived from iPSCs specific to patients with TS (Extended Data Fig. 5k-m). Because loss-of-function mutations 

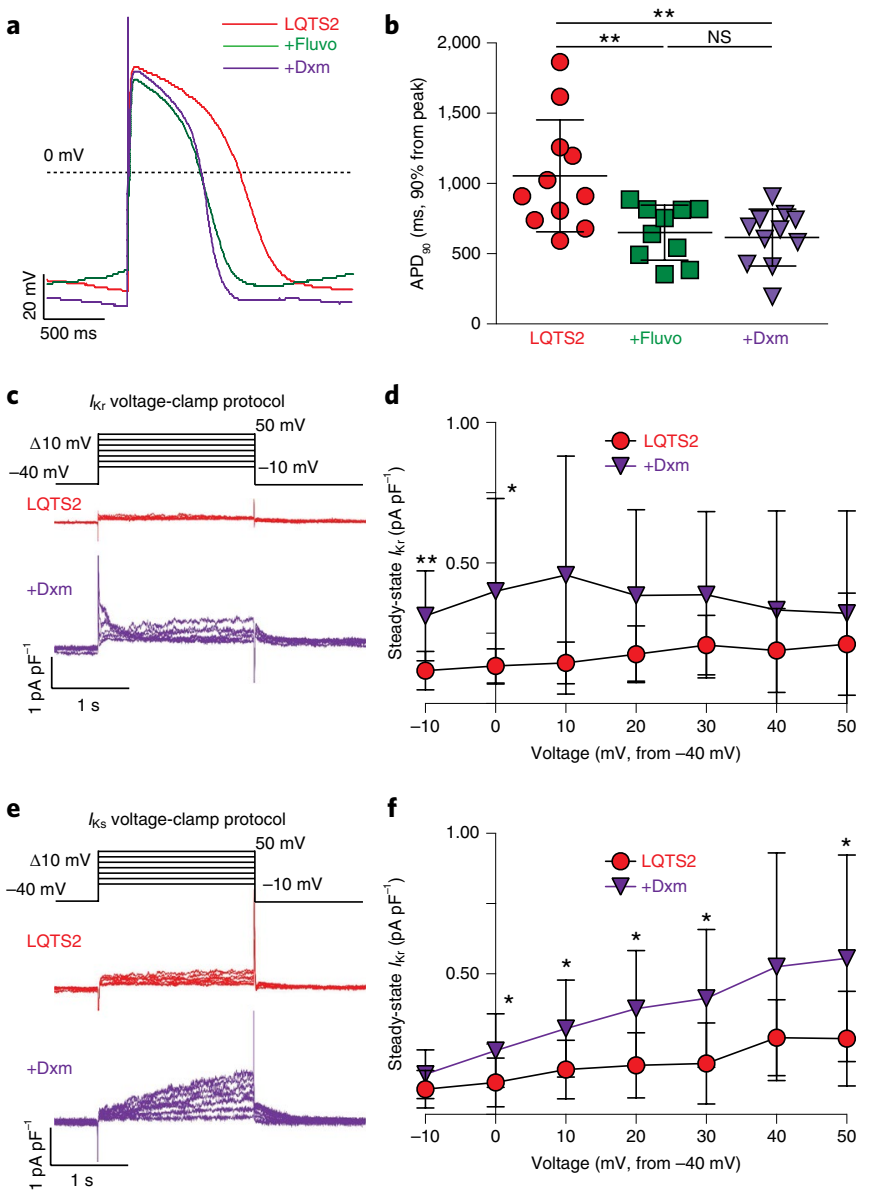

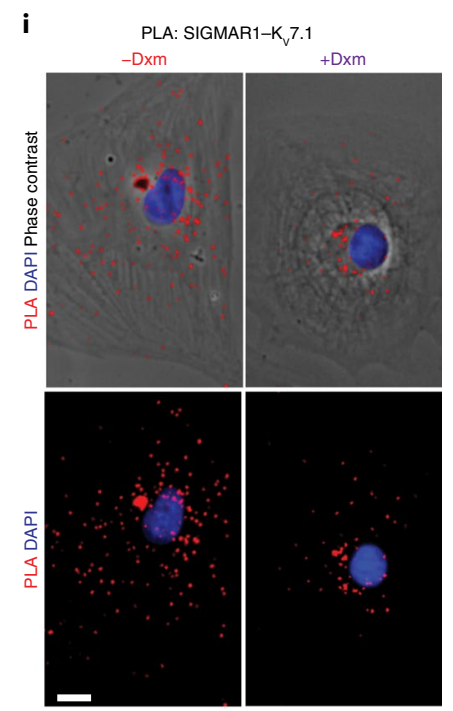

Fig. 4 | The effect of dextromethorphan on phenotypes in LQTS2. a, Representative traces of action potentials in cardiomyocytes derived from iPSCs of patients with LQTS2 without treatment or treated with fluvoxamine or dextromethorphan. $\mathbf{b}$, APD $_{90}$ analysis of cardiomyocytes from patients with LQTS2 without treatment $(n=11)$ or treated with fluvoxamine $(n=10)$ or dextromethorphan $(n=11)$. Other action potential parameters are shown in Extended Data Fig. 7b,d,f. c, Representative traces of $I_{\mathrm{Kr}}$ currents (E-4031 sensitive) in cardiomyocytes from patients with LQTS2 treated with dextromethorphan or untreated. d, $I_{\mathrm{Kr}}$ currents (steady state) were significantly increased in dextromethorphan-treated cardiomyocytes from patients with LQTS2 ( $n=9$ ) at $-10-m V$ and $0-m V$ steps compared with those of cardiomyocytes from patients with LQTS2 without treatment $(n=10)$. e, Representative traces of $I_{\mathrm{Ks}}$ currents (chromanol 293B sensitive) in cardiomyocytes from patients with LQTS2 treated with dextromethorphan or untreated. $\mathbf{f}, I_{\mathrm{Ks}}$ currents (steady state) were significantly increased in dextromethorphan-treated cardiomyocytes from patients with LQTS2 at 0-, 10-, 20-, 30- and 50-mV steps compared with those in cardiomyocytes from patients with LQTS2 without treatment ( $n=10$ per group). $\mathbf{g}$, Representative epifluorescent and phase-contrast images of cardiomyocytes from patients with LQTS2 with and without dextromethorphan treatment from the PLA (SIGMAR1-hERG, red; DAPI, blue). Scale bar, $10 \mu \mathrm{m}$. h, Quantification of SIGMAR1-hERG by the PLA in dextromethorphan-treated $(n=22)$ and untreated $(n=29)$ cardiomyocytes from patients with LQTS2. i, Representative epifluorescent and phase-contrast images of cardiomyocytes from patients with LQTS2 with and without dextromethorphan treatment from the PLA (SIGMAR1-K 7.1, red; DAPI, blue). Scale bar, $10 \mu \mathrm{m}$. j, Quantification of SIGMAR1-K 7.1 by the PLA in dextromethorphan-treated $(n=107)$ and untreated $(n=87)$ cardiomyocytes from patients with LQTS2. All data are mean \pm s.d. Treatment with fluvoxamine or dextromethorphan was carried out at $5 \mu \mathrm{M}$ for $2 \mathrm{~h}$. One-way ANOVA with Tukey's multiple comparisons was used for $\mathbf{b}$. Unpaired two-tailed Student's $t$-tests was used for $\mathbf{h}, \mathbf{j}$ between groups and for $\mathbf{d}, \mathbf{f}$ at each voltage step. ${ }^{\star} P<0.05,{ }^{\star \star} P<0.01,{ }^{\star \star \star} P<0.001$. Cardiomyocytes were from at least two independent differentiations.

in $\mathrm{K}_{\mathrm{V}} 7.1$ are also known to cause LQTS1 and QT prolongation, we hypothesized that the increase in $\mathrm{K}_{\mathrm{V}} 7.1$ expression might contribute to the beneficial effect of dextromethorphan on the prolonged QT interval in the mouse model of TS. To validate proteomic results, we examined the expression of $\mathrm{K}_{\mathrm{V}} 7.1$ as well as that of house-keeping molecules $\beta$-tubulin and glyceraldehyde-3-phosphate dehydrogenase (Gapdh) using western blotting and mouse heart lysates. We found that, consistent with proteomic results (Extended Data Fig. 9), the expression of $\beta$-tubulin significantly increased in mouse hearts in the TS model compared with that in other groups, while no changes in the expression of Gapdh protein between the groups were observed (Extended Data Fig. 10a,b). Also in line with proteomic results and our observation using cardiomyocytes from patients with TS, there was a significant reduction in $\mathrm{K}_{\mathrm{v}} 7.1$ protein expression in mouse hearts in the TS model, while dextromethorphan treatment restored the expression of $\mathrm{K}_{\mathrm{V}} 7.1$ to a level comparable to that of control hearts (Extended Data Fig. 10a,c). When we examined changes in Kcnq1 transcripts in mouse hearts, dextromethorphan treatment did not significantly upregulate Kcnq1 transcription in hearts in the TS model, although hearts in the TS model showed a trend toward reduced Kcnq1 gene expression compared with control hearts (Extended Data Fig. 10d). Overall, the results suggest that the significant reduction in $\mathrm{K}_{\mathrm{V}} 7.1$ protein levels in hearts in the TS model may be associated with QT prolongation, although the contribution of $\mathrm{K}_{\mathrm{v}} 7.1$ to cardiac action potential repolarization in rodents is known to be relatively smaller than that in humans, and dextromethorphan might alleviate cardiac phenotypes in the mouse model of TS in part by increasing 


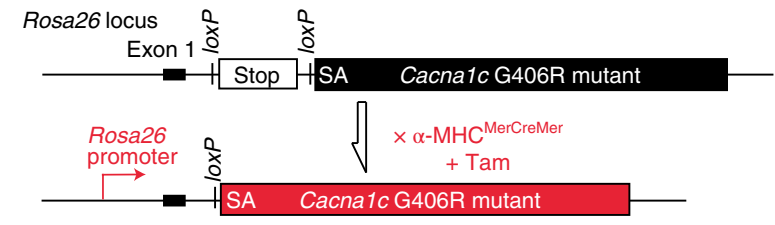

b 12 -week-old Rosa26 $6^{\mathrm{GR}^{+}} ; \alpha-\mathrm{MHC}^{\mathrm{MerCreMer}}{ }^{+}$mice

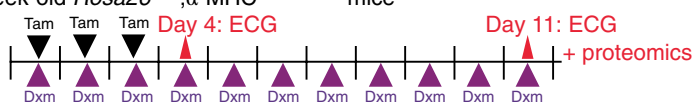

C

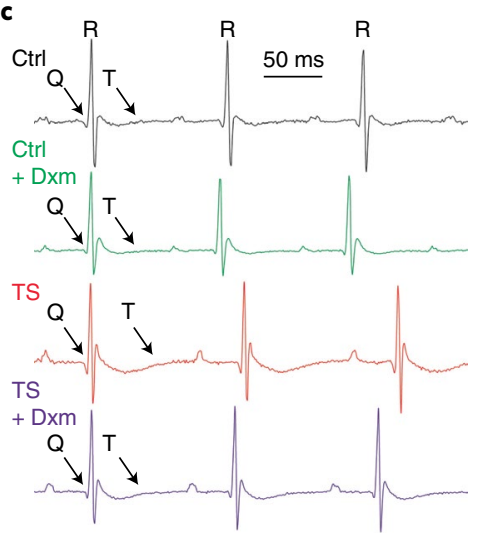

d

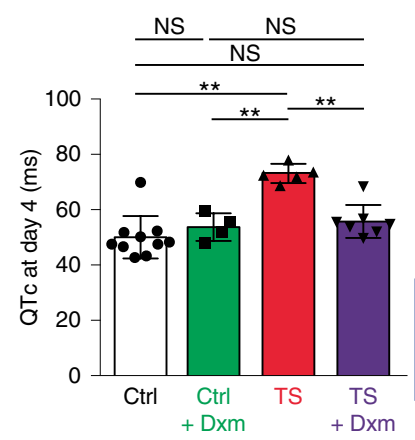

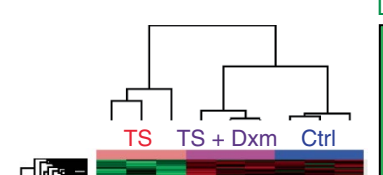

ow in TS
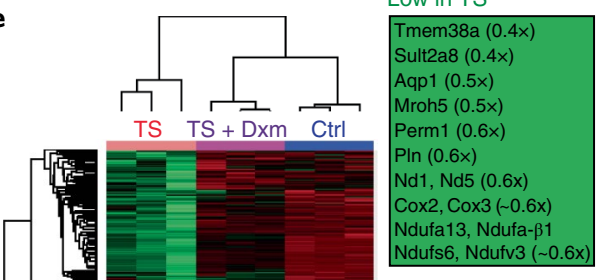

High in TS

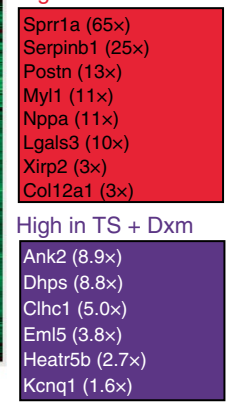

Upstream

Tp53 Tgf- $\beta$

Cardiotoxicity

Damage

Fig. 5 | The effect of dextromethorphan on the mouse model of TS. a, Targeting design of a TS mutant (corresponding to G406R in the Ca2+ channel Cacna1c gene) at the mouse Rosa26 locus and the breeding strategy for inducing cardiac-specific tamoxifen (Tam)-inducible expression of TS mutant channels. SA, splice acceptor. b. Experimental design for the induction of TS mutant channel expression in the heart with tamoxifen and testing dextromethorphan in vivo. Dextromethorphan was added to drinking water, and pharmacokinetic analyses of plasma dextromethorphan concentrations at day 4 and day 11 are shown in Extended Data Fig. 8a. c, Representative ECG traces in the mouse model of TS (TS) and control littermates (Ctrl) without treatment or treated with dextromethorphan at day 4. d, Prolonged QT intervals in the mouse model of TS were normalized by dextromethorphan treatment ( $n=10$ for control littermates, $n=4$ for control littermates treated with dextromethorphan, $n=5$ for TS, $n=7$ for TS and dextromethorphan). QT intervals were corrected using a mouse formula (QTc, Extended Data Fig. 8b). One-way ANOVA with Sidak's multiple comparisons was used ( $* \star P<0.01$ ). e, Proteomic analysis including heat map comparisons and pathway analysis at day 11 in hearts of control mice, untreated mice and dextromethorphantreated mice in the TS model ( $n=3$ per group). ECG at day 11 also confirmed the beneficial effect of dextromethorphan on QT prolongation in mutant mice (Extended Data Fig. 8c). Additional analyses of several proteins with altered expression levels are shown in Extended Data Fig. 9. All data are mean \pm s.d.

expression of $\mathrm{K}_{\mathrm{V}} 7.1$ in hearts in the TS model through a post-transcriptional mechanism.

\section{Discussion}

In summary, our results demonstrate that SIGMAR1 agonists such as dextromethorphan can rescue the phenotypes in cardiomyocytes derived from iPSCs of patients with TS and the mouse model of TS as well as cardiomyocytes derived from iPSCs of patients with LQTS1 or LQTS2 with the G269S substitution in the $\mathrm{K}_{\mathrm{V}} 7.1$ channel or the A561V substitution in the hERG channel, respectively. These results indicate that SIGMAR1 could be a therapeutic target for TS as well as LQTS1 and LQTS2, which are caused by trafficking mutations such as those corresponding to the G269S substitution in the $\mathrm{K}_{\mathrm{V}} 7.1$ channel and the A561V substitution in the hERG channel. Furthermore, we explored the underlying mechanisms of the beneficial effects of SIGMAR1 activation on cardiomyocytes from patients with TS and found that SIGMAR1 may play a multifaceted role in regulating $\mathrm{Ca}_{\mathrm{V}} 1.2$ channel function in cardiomyocytes. First, SIGMAR1 directly interacts with CDK5, and SIGMAR1 activation induces a significant increase in the interaction and a significant reduction in CDK5 protein expression and activity in cardiomyocytes from patients with TS, which may restore the affected voltage-dependent inactivation of $\mathrm{Ca}_{\mathrm{v}} 1.2$ in patient cardiomyocytes through the mechanism that we proposed previously ${ }^{6}$ and rescue cellular phenotypes (Fig. 1 and Extended Data Figs. 1 and 2). In addition, SIGMAR1 also directly interacts with $\mathrm{Ca}_{\mathrm{V}} 1.2$ in cardiomyocytes from patients with TS, and the activation of SIGMAR1 induced by dextromethorphan treatment modulates the interaction between SIGMAR1 and $\mathrm{Ca}_{\mathrm{V}} 1.2$ (Extended Data Fig. 3h,i), which may also contribute to the beneficial effects of SIGMAR1 activation on cardiomyocytes from patients with TS. Our findings provide insights into the interactions between SIGMAR1, CDK5 and $\mathrm{Ca}_{\mathrm{V}} 1.2$ in human iPSC-derived cardiomyocytes, while detailed molecular mechanisms concerning the interactions remain to be fully characterized. Moreover, it is also of merit to further investigate whether other players, such as calmodulin and calmodulin-dependent protein kinase II, which play important roles in regulating $\mathrm{Ca}^{2+}$ channel function ${ }^{43}$, or the $\mathrm{Na}^{+} / \mathrm{Ca}^{2+}$ exchanger, which is known to modulate $\mathrm{Ca}^{2+}$ homeostasis in cardiomyocytes ${ }^{44}$, may interact with SIGMAR1 and mediate the effects of SIGMAR1 activation on $\mathrm{Ca}^{2+}$ channel and ion homeostasis.

Unexpectedly, we found that there was a significant reduction in both $I_{\mathrm{Kr}}$ and $I_{\mathrm{Ks}}$ currents in cardiomyocytes from patients with TS compared to those of isogenic control cardiomyocytes (Extended Data Figs. 3j,k and 5f), although our iPSC lines from patients with TS do not carry any mutations in KCNH2 (hERG) or KCNQ1 $\left(\mathrm{K}_{\mathrm{V}} 7.1\right)$. More interestingly, we found that, in addition to its effects on the $\mathrm{Ca}_{\mathrm{V}} 1.2$ channel, activation of SIGMAR1 by dextromethorphan also increased $\mathrm{K}^{+}$currents in cardiomyocytes from patients with TS (Fig. 2 and Extended Data Figs. 3 and 5), which may further contribute to the normalization of action potentials and restoration of ion homeostasis in cardiomyocytes from patients with TS. 
Mechanistically, we observed that SIGMAR1 interacted with hERG and $\mathrm{K}_{\mathrm{V}} 7.1$ channels in human iPSC-derived cardiomyocytes, and dextromethorphan treatment modulated the interactions between SIGMAR1, hERG and the $\mathrm{K}_{\mathrm{V}} 7.1$ channel, increased the plasma membrane localization of $\mathrm{K}_{\mathrm{V}} 7.1$ and increased gene and protein expression of KCNQ1 and $\mathrm{K}_{\mathrm{V}} 7.1$ isoform 1 in cardiomyocytes from patients with TS. Although, to our knowledge, the interaction between SIGMAR1 and $\mathrm{K}_{\mathrm{V}} 7.1$ has not been reported previously, the direct interaction between SIGMAR1 and hERG has been studied in leukemic cells, HEK cells and neonatal rat cardiomyocytes ${ }^{12,32-34}$. Our results confirmed the direct interaction between SIGMAR1 and the hERG channel and SIGMAR1-mediated regulation of hERG channel function in human iPSC-derived cardiomyocytes, while detailed molecular mechanisms remain to be fully characterized. Additionally, dextromethorphan has been previously reported to inhibit hERG currents in a heterologous overexpression system ${ }^{45}$, while we found that dextromethorphan treatment significantly increased hERG currents in human patient-specific iPSC-derived cardiomyocytes. There have been notable differences in hERG currents in human iPSC-derived cardiomyocytes ${ }^{46}$ and heterologous systems potentially due to differences in cellular physiological conditions and associated molecules. Further investigations are needed to profile the effects and mechanisms of dextromethorphan and SIGMAR1 activation on hERG and $\mathrm{K}_{\mathrm{V}} 7.1$ channels.

By contrast, when we examined the effects of dextromethorphan on electrophysiological properties of isogenic control cardiomyocytes, no significant changes were observed in action potentials (Fig. 1), $\mathrm{Ca}_{\mathrm{V}} 1.2$ inactivation (Extended Data Fig. 3) or $\mathrm{K}^{+}$currents (Extended Data Fig. 4) in isogenic control cardiomyocytes treated with dextromethorphan at a dose that was efficacious for cardiomyocytes from patients with TS. We sought to understand the difference between the sensitivity of cardiomyocytes from patients with TS and isogenic control cardiomyocytes to the SIGMAR1 agonist dextromethorphan, and we found that, at baseline, there was a significant increase in the expression of SIGMAR1 transcripts and SIGMAR1 protein in cardiomyocytes from patients with TS (Extended Data Fig. 2). Furthermore, we examined the expression of ATF4, a transcription factor that was reported to directly regulate SIGMAR1 transcription ${ }^{24}$, and observed an increase in ATF4 protein levels in cardiomyocytes from patients with TS, which may contribute to the increased expression of SIGMAR1 in cardiomyocytes from patients with TS. Overall, the results suggest that cardiomyocytes from patients with TS have increased SIGMAR1 expression at baseline, which may predispose cells to respond to SIGMAR1 agonists. In addition, it is also likely that, compared to cardiomyocytes from patients with TS with highly disrupted ion homeostasis, normal cardiomyocytes without any mutations in genes encoding cardiac ion channels have a higher capacity to tolerate moderate changes in ion channel function and maintain stable ion homeostasis, which contributes to the lack of changes observed in isogenic control cardiomyocytes with dextromethorphan treatment.

The data from cardiomyocytes of patients with TS encouraged us to test SIGMAR1 agonists on cardiomyocytes from patients with LQTS1 or LQTS2 with trafficking mutations. Cardiomyocytes from patients with LQTS1 and the trafficking mutation corresponding to G269S and cardiomyocytes from patients with LQTS2 and the trafficking mutation corresponding to $\mathrm{A} 561 \mathrm{~V}$ were reported to have decreased $\mathrm{K}^{+}$current $^{35,36}$, and we hypothesized that SIGMAR1 activation might be useful to restore $\mathrm{K}^{+}$currents and alleviate their cellular phenotypes. Consistent with our hypothesis, cardiomyocytes from patients with LQTS1 and the G269S substitution and cardiomyocytes from patients with LQTS2 and the A561V substitution did demonstrate a decrease in $\mathrm{K}^{+}$currents, and dextromethorphan treatment rescued the cellular phenotypes in cardiomyocytes from patients with LQTS1 or LQTS2 (Figs. 3 and 4 and Extended Data Figs. 6 and 7) by modulating interactions between SIGMAR1,
hERG and the $\mathrm{K}_{\mathrm{V}} 7.1$ channel and increasing $\mathrm{K}^{+}$currents. Our results demonstrated that SIGMAR1 activation could be beneficial for not only TS but also for LQTS1 and LQTS2, which are caused by the G269S substitution in the $\mathrm{K}_{\mathrm{V}} 7.1$ channel and the A561V substitution in the hERG channel, respectively. However, the effect of SIGMAR1 agonists and SIGMAR1 activation on LQTS1 and LQTS2 with other mutations remains to be investigated.

Intriguingly, in addition to cardiomyocytes derived from human iPSCs from patients with TS, the SIGMAR1 agonist and FDAapproved cough suppressant dextromethorphan also exhibited beneficial effects in the mouse model of TS (Fig. 5 and Extended Data Figs. 8-10). While dextromethorphan was previously reported to have a protective effect on ouabain-induced acute arrhythmias ${ }^{47}$, we reported that dextromethorphan treatment could be beneficial in a mouse model of inherited cardiac arrhythmia (TS). One of the limitations of our study is that we did not examine the effect of dextromethorphan on LQTS1 or LQTS2 mouse models due to the lack of an LQTS1 or LQTS2 mouse model recapitulating arrhythmic phenotypes in vivo ${ }^{38}$, and additional studies are necessary to evaluate the potential effect of dextromethorphan on LQTS1 and LQTS2 in vivo. Furthermore, it is also worth noting that dextromethorphan has several limitations related to clinical applications: dextromethorphan has a short half-life and induces nausea, which may limit its tolerability and long-term use, overdose of dextromethorphan could potentially lead to severe adverse cardiac events ${ }^{48,49}$, and the pharmacological profile of dextromethorphan in infants and young children has not been fully investigated, which may comprise the main demographic of patients with TS. Therefore, further investigations are absolutely required to evaluate whether dextromethorphan can be repurposed to alleviate cardiac arrhythmias in patients with TS. In addition, TS is a multiorgan disorder that features not only cardiac arrhythmias but also syndactyly, autism and hypoglycemia because of the important role of the $\mathrm{Ca}_{\mathrm{V}} 1.2$ channel in various organs $s^{1,3,37,50}$. Therefore, the effects of dextromethorphan on the dysfunction of other affected organs remain to be investigated.

In conclusion, although the role of SIGMAR1 has emerged in various diseases including neurological disorders and cancer ${ }^{9-11}$ and more recently in cardiovascular disease ${ }^{51,52}$ and coronavirus disease 2019 (refs. ${ }^{53,54}$ ), our findings demonstrate that SIGMAR1 could be a therapeutic target for TS and potentially LQTS1 and LQTS2, which are caused by the G269S substitution in the $\mathrm{K}_{\mathrm{V}} 7.1$ channel and the A561V substitution in the hERG channel, respectively. Further investigations are necessary to profile global effects of SIGMAR1 activation and its agonists on different organs and tissues and to develop and optimize SIGMAR1 agonists for future clinical applications.

\section{Methods}

Ethical statement. All animal experimental procedures were carried out in accordance with regulations and established guidelines and were reviewed and approved by the Institutional Animal Care and Use Committee at Columbia University (AC-AAAU2453).

Human iPSC culture and cardiac cell differentiation. iPSC lines from patients with TS and their corresponding isogenic control iPSC lines were generated and described previously ${ }^{6,55}$. Briefly, iPSC lines from patients with TS were generated with Lipofectamine LTX (Thermo Fisher Scientific) and three episomal vectors (Addgene, 27077, 27078 and 27080) using skin fibroblasts isolated from patients with TS. Their corresponding isogenic control iPSCs were generated using Lipofectamine LTX and plasmids encoding the transcription activator-like effector nuclease (TALEN) from patient-specific iPSC lines, targeting the CACNA1C gene (exon 8a). iPSC lines from patients with LQTS1 and LQTS2 were generated in J. Wu's laboratory at Stanford University and shared with us (Stanford Cardiovascular Institute, SCVI22 and SCVI498 lines, respectively). Isogenic control iPSC lines were generated from the iPSC lines derived from patients with LQTS1 and LQTS2 using gene-editing technology as reported in the current study. Human iPSCs were cultured in Essential 8 medium (Thermo Fisher Scientific) with $100 \mathrm{U} \mathrm{ml}^{-1}$ penicillin and $100 \mu \mathrm{g} \mathrm{ml}^{-1}$ streptomycin (PS) on plates or dishes (Corning) coated with Geltrex (Thermo Fisher Scientific) following 
the manufacturer's instructions. Dispase (Thermo Fisher Scientific) dissolved in the culture medium was used for cell dissociation or passaging. iPSC lines were differentiated into cardiomyocytes following a monolayer-based protocol that we described previously ${ }^{55}$. CHIR99021 (CHIR, GSK3 inhibitor, 1386, Axon Medchem; the dose used for differentiation was titrated for each iPSC line, and the dose range is $0.25-5 \mu \mathrm{M}$ ) and BIO (GSK3 inhibitor IX, 361550, Calbiochem) and IWP-3 (WNT inhibitor, SML0533, Sigma-Aldrich) dissolved in dimethyl sulfoxide (DMSO, 276855, Sigma-Aldrich) were used for monolayer differentiation of iPSCs plated on Geltrex in six-well plates or 10-cm dishes. Only one freeze-thaw cycle was tested for all aliquots of the compounds CHIR and BIO. Two freeze-thaw cycles were tested for IWP-3 aliquots. For the differentiation protocol, DF20/5 medium (DMEM/F-12 (with GlutaMAX I) containing $20 \%$ or $5 \%$ HyClone fetal bovine serum (FBS), 1\% MEM non-essential amino acids, PS and $0.1 \mathrm{mM}$ 2-mercaptoethanol) was used for cardiac differentiation and culture. Additional D-glucose was added to only DF20 medium to create high-glucose medium (final concentration of $4.5 \mathrm{gl}^{-1}$ ) to enhance mesoderm differentiation from day 1 to day 4. Treatment with BIO $(1 \mu \mathrm{M})$ and CHIR (at the dose optimized for each line) was carried out at days $2-4$. The medium with BIO and CHIR was changed every day. Treatment with $2 \mu \mathrm{M}$ IWP-3 was carried out at days 5-11, and the medium was changed every other day. At days 5-7, DF20 medium was used with IWP-3. DF5 medium was used on day 7 for IWP-3 treatment and maintenance, and the medium was changed every other day. Changing medium with the compounds required $\sim 3 \mathrm{ml}$ per well in six-well plates and $\sim 15 \mathrm{ml}$ per $10-\mathrm{cm}$ dish every other day because adding $2-2.5 \mathrm{ml}$ fresh medium to a well of a six-well plate was not sufficient for high confluence of cells during differentiation into cardiomyocytes, resulting in lower efficiency of cardiac differentiation. Several lots of USDAapproved FBS (from Thermo Fisher, HyClone and Gibco) were tested after we found that CHIR and BIO in DMEM/F-12 was compatible with FBS, and HyClone FBS was only used for further monolayer differentiation because we found that this lot of HyClone FBS provided slightly higher efficiency in generating cardiomyocytes reproducibly than other FBS lots.

Gene editing to generate isogenic controls. A modification of a previously described ${ }^{56}$ RNP-based CRISPR-Cas9 editing protocol was used. Briefly, singlestranded guide RNA (sgRNA) and PAM sites were identified by using a freely available online tool (https://design.synthego.com/\#/). Donors (ssODN) were designed manually following incorporation of the desired nucleotide and silencing of the PAM sequence. GENECODE release 26 (GRCh38.p10) was used as reference sequence for each target. Electroporation was performed using the P3 Primary Cell 4D-Nucleofector X Kit S (Lonza, V4XP-3032) and the Lonza 4D-nucleofector according to the manufacturer's instructions. The RNP complex was prepared by adding $3 \mu \mathrm{g}$ sgRNA (Integrated DNA Technologies, IDT) to $3 \mu \mathrm{g}$ ssODN (IDT) and $2.5 \mu \mathrm{g}$ Cas9 protein (Alt-R S.p. HiFi Cas9 Nuclease V3, IDT) in P3 electroporation buffer. The solution was incubated for $30 \mathrm{~min}$ at room temperature to allow formation of the RNP complex. Human iPSCs (LQTS1 and LQTS2 lines) were detached using warm Accutase (Stemcell Technologies) and washed with $\mathrm{Ca}^{2+}$. and $\mathrm{Mg}^{2+}$-free PBS, and then $1 \times 10^{6}$ cells were resuspended in the electroporation complex and electroporated using the program CA150. Low-density seeding $\left(3 \times 10^{3}\right.$ cells per $10-\mathrm{cm}$ dish) was performed to grow single-cell colonies. Electroporated cells were allowed to grow for $48 \mathrm{~h}$ on Matrigel (Corning)-coated 96-well plates with mTeSR Plus medium (Stemcell Technologies) supplemented with $10 \%$ CloneR (Stemcell Technologies) at $37^{\circ} \mathrm{C}$ with $5 \% \mathrm{CO}_{2}$. Colonies were manually picked in 96-well plates, and genotyping was performed by genomic DNA isolation, PCR amplification of the target region and subsequent Sanger sequencing (primers from Genewiz, Taq polymerase from Takara Bio). Edited clones were further expanded and tested for karyotypic abnormalities (Columbia University Genetics laboratory). sgRNA, ssODN and primer information are shown as follows: KCNQ1 (sgRNA3, AGCCAGGTACACAAAGTACG PAM AGG; ssODN3, GCCTAGGAGCTGATAACCACCCTGTACATCgGCTTCCTGGGCC TCATCTTCTC'g'TCGTACTTTGTGTACCTGGCTGAGAAGGACGCGGTGA ACGAGTCA (g, target corrected A to g; 'g', PAM silence C to g); primers (PCR template size, $560 \mathrm{bp}$ ): KCNQ1F1 (GCTATATTGAAGCCGGCCCT, amplification), KCNQ1R1 (CCAGTGACCCCTCCGATTTC, amplification), KCNQ1F2 (CTTAGGCGTCTGCACAGGAG, sequencing)), KCNH2 (sgRNA3, GCCTGCATCTGGTACGCCAT PAM CGG; ssODN3, GTTCTTGCTCATGTG CACCTTTGCGCTCATCGcGCACTGGCTAGCCTGCATCTGGTACGCCAT CG't'CAACATGGAGCAGCCACACATGGACTCACGCATCGGCT (c, target corrected $\mathrm{T}$ to $\mathrm{C}$; ' $\mathrm{t}$ ', PAM silence $\mathrm{G}$ to $\mathrm{t}$ ); primers (PCR template size, $388 \mathrm{bp}$ ): KCNH2F1 (CAGTGTGGGCTTCACCTCTTAG, amplification), KCNH2R1 (TGCTGTTGTAGGGTTTGCCTATC, amplification), KCNH2F2 (GGATCTGA CCTCTGATGCTCG, sequencing)).

Preparation of chemical compounds and binding modeling. Fluvoxamine maleate (F2802, Sigma-Aldrich), PRE-084 hydrochloride (0589, Tocris), dextromethorphan (D2531, Sigma-Aldrich) and NE-100 hydrochloride (3133, Tocris) were dissolved in distilled water to make stock solutions with a concentration of $10 \mathrm{mM}$ or $25 \mathrm{mM}$. Stock solutions were stored at $-80^{\circ} \mathrm{C}$ and thawed only once for in vitro experiments. The putative binding of these agonists to SIGMAR1 was obtained using known templates in SWISS-MODEL
(Biozentrum) that produced a high identity with human SIGMAR1 structure $(5 \mathrm{HK} 1 \text { and } 5 \mathrm{HK} 2)^{14}$. This was used to generate docking models that were visualized using CueMol2.

Patch-clamp recording. Human iPSC-derived cardiomyocytes were dissociated into single cells for whole-cell patch-clamp recordings 1-2 months after cardiac differentiation was induced, and the dissociated cells were used for patch-clamp recording 4-8 d after dissociation, following a protocol described previously ${ }^{6}$. Whole-cell patch-clamp recordings of iPSC-derived cardiomyocytes were conducted using a MultiClamp 700B patch-clamp amplifier (Molecular Devices) and an inverted microscope equipped with differential interface optics (Nikon, Ti-U). Glass pipettes were prepared using borosilicate glass (Sutter Instrument, BF150-110-10) and a micropipette puller (Sutter Instrument, model P-97). Voltageclamp measurements for voltage-dependent $\mathrm{Ca}^{2+}$ channel inactivation were carried out using an extracellular solution consisting of $5 \mathrm{mM} \mathrm{BaCl}_{2}, 160 \mathrm{mM}$ TEA and $10 \mathrm{mM}$ HEPES ( $\mathrm{pH} 7.4$ at $25^{\circ} \mathrm{C}$ ) and a pipette solution consisting of $125 \mathrm{mM}$ $\mathrm{CsCl}, 0.1 \mathrm{mM} \mathrm{CaCl}_{2}, 10 \mathrm{mM}$ EGTA, $1 \mathrm{mM} \mathrm{MgCl}, 4 \mathrm{mM}$ magnesium ATP and $10 \mathrm{mM}$ HEPES ( $\mathrm{pH} 7.4$ with $\mathrm{CsOH}$ at $25^{\circ} \mathrm{C}$ ). For $\mathrm{Ca}^{2+}$ current recordings, another extracellular solution was used instead: $5 \mathrm{mM} \mathrm{CaCl}_{2}, 137 \mathrm{mM} \mathrm{NMDG}, 0.5 \mathrm{mM}$ $\mathrm{MgCl}_{2}, 25 \mathrm{mM} \mathrm{CsCl}, 10 \mathrm{mM}$ TEA chloride, $10 \mathrm{mM}$ glucose and $10 \mathrm{mM}$ HEPES $\left(\mathrm{pH} 7.4\right.$ with $\mathrm{CsOH}$ at $\left.25^{\circ} \mathrm{C}\right)$. Cells were held at $-90 \mathrm{mV}$ and then at $-50 \mathrm{mV}$ (2-s hold) and stimulated with pulses from $-60 \mathrm{mV}$ to $+20 \mathrm{mV}$ for the current-voltage relationship of $\mathrm{Ba}^{2+}$ currents. Recordings were conducted at room temperature. Voltage-clamp recording for $I_{\mathrm{Kr}}$ measurements was conducted in normal Tyrode's solution containing $140 \mathrm{mM} \mathrm{NaCl}, 5.4 \mathrm{mM} \mathrm{KCl}, 1 \mathrm{mM} \mathrm{MgCl}_{2}, 10 \mathrm{mM}$ glucose, $1.8 \mathrm{mM} \mathrm{CaCl}_{2}$ and $10 \mathrm{mM}$ HEPES (pH 7.4 with $\mathrm{NaOH}$ ) at $37^{\circ} \mathrm{C}$ supplemented with $0.5 \mu \mathrm{M}$ nisoldipine ( $I_{\mathrm{Ca}}$ current blocker, Sigma-Aldrich, N0165) and $5 \mu \mathrm{M}$ (-)-[3R,4S]-chromanol 293B $\left(I_{\mathrm{Ks}}\right.$ current blocker, Tocris, 1475) using the pipette solution $120 \mathrm{mM}$ potassium D-gluconate, $25 \mathrm{mM} \mathrm{KCl}, 4 \mathrm{mM}$ magnesium ATP, $2 \mathrm{mM}$ sodium GTP, $4 \mathrm{mM}$ phosphocreatine disodium, $10 \mathrm{mM}$ EGTA, $1 \mathrm{mM} \mathrm{CaCl}_{2}$ and $10 \mathrm{mM}$ HEPES ( $\mathrm{pH} 7.4$ with $\mathrm{KCl}$ at $25^{\circ} \mathrm{C}$ ). The following pulse protocol was used: a 2-s voltage clamp was applied from $-10 \mathrm{mV}$ to $50 \mathrm{mV}(\Delta 10 \mathrm{mV}$, holding at $-40 \mathrm{mV}$ and $0.1 \mathrm{~Hz}$ at $37^{\circ} \mathrm{C}$ ). All components used for the above recording solutions were purchased from Sigma-Aldrich except (-)-[3R,4S]-chromanol 293B (Tocris). After recordings, the extracellular solution was exchanged for one containing $1 \mu \mathrm{M}$ E-4031 (Tocris, 1808) using perfusion. The E-4031-sensitive current was measured and analyzed as $I_{\mathrm{Kr}}$ current using Clampfit 10.4 (Axon Instruments). For $I_{\mathrm{Ks}}$ current recordings, instead of (-)-[3R,4S]-chromanol 293B, E-4031 $(0.5 \mu \mathrm{M})$ was used for base recordings, and then $(-)-[3 R, 4 S]$-chromanol $293 \mathrm{~B}(10 \mu \mathrm{M})$ was applied to measure $(-)-[3 R, 4 S]$-chromanol 293B-sensitive currents as $I_{\mathrm{Ks}}$. Recordings were conducted at $37^{\circ} \mathrm{C}$

Current-clamp recordings were conducted in normal Tyrode's solution containing $140 \mathrm{mM} \mathrm{NaCl}, 5.4 \mathrm{mM} \mathrm{KCl}, 1 \mathrm{mM} \mathrm{MgCl}_{2}, 10 \mathrm{mM}$ glucose, $1.8 \mathrm{mM}$ $\mathrm{CaCl}_{2}$ and $10 \mathrm{mM} \mathrm{HEPES}$ ( $\mathrm{pH} 7.4$ with $\mathrm{NaOH}$ at $25^{\circ} \mathrm{C}$ ) using the pipette solution $120 \mathrm{mM}$ potassium D-gluconate, $25 \mathrm{mM} \mathrm{KCl}, 4 \mathrm{mM}$ magnesium ATP, $2 \mathrm{mM}$ sodium GTP, $4 \mathrm{mM}$ phosphocreatine disodium, $10 \mathrm{mM} \mathrm{EGTA,} 1 \mathrm{mM} \mathrm{CaCl}_{2}$ and $10 \mathrm{mM}$ HEPES ( $\mathrm{pH} 7.4$ with $\mathrm{KCl}$ at $25^{\circ} \mathrm{C}$ ). Recordings were conducted at $37^{\circ} \mathrm{C}$. Cardiac action potentials were stimulated $(2 \mathrm{~ms}, 1 \mathrm{nA}, 0.2 \mathrm{~Hz}$ for lines from patients with TS or $0.5 \mathrm{~Hz}$ for other lines) in current-clamp mode at $37^{\circ} \mathrm{C}$. Recorded action potentials were analyzed using Clampfit 10.4. Control recordings were obtained from cardiomyocytes without treatment. After-treatment recordings were obtained from cardiomyocytes that had been treated with either dextromethorphan, fluvoxamine or PRE-084 at a dose of $5 \mu \mathrm{M}$ for $2 \mathrm{~h}$ in the cardiomyocyte culture medium that we described previously ${ }^{6,55}$. The dose of $5 \mu \mathrm{M}$ was empirically chosen for the compounds based on our previous studies. Patch-clamp data were acquired and analyzed using pCLAMP 10 (Molecular Devices) and Clampfit 10.4 (Axon Instruments).

Cardiomyocyte contraction analysis. The working solution of the SIGMAR1 agonist PRE-084 was made by diluting the stock solution in cardiomyocyte medium to a final concentration of $5 \mu \mathrm{M}$ PRE-084. Contraction analysis was performed as reported previously ${ }^{5,6}$. In brief, images were collected at a rate of five frames per second using NIS-Elements software (4.51.01, Nikon) and converted to multi-frame TIFF images for analysis using the NIS-Elements viewer. Multiple-frame TIFF images were processed using the Image Processing Toolbox in MATLAB R2009b (MathWorks). Relative motion between successive frames was quantified by subtracting each frame from the preceding frame and summing across all pixels. Movement was calculated by plotting the relative motion over time. Contractions were detected as a peak of relative motion, and a second, typically smaller peak, corresponded to the relaxation of cardiomyocytes. Peaks were selected manually, and the rate of contraction was measured. The length of time between contractions was also measured, and contraction irregularity was measured by calculating the ratio of the standard deviation to the mean of the intervals between contractions. Cardiomyocytes from patients with TS were used for tests on days 21-22 after cardiac differentiation. Movies were recorded before treatment and $2 \mathrm{~h}$ after treatment with PRE-084 from the intact monolayer of cardiomyocytes from patients with TS. Cardiomyocytes from two iPSC lines from patients with TS (clones TS1-E3-5 and TS2-E7-1) were used for this contraction assay. The contraction rate and the irregularity of each sample before and after 
treatment were compared using paired two-tailed Student's $t$-tests. The contraction rate and irregularity value of each sample after treatment were normalized to their respective values before treatment for the corresponding figures.

Calcium imaging. For $\mathrm{Ca}^{2+}$ transient recordings to confirm the phenotype of cardiomyocytes from patients with TS compared with that of control cardiomyocytes (shown in Extended data Fig. 3) and to examine the effects of dextromethorphan on abnormal spontaneous $\mathrm{Ca}^{2+}$ transients in cardiomyocytes from patients with TS (shown in Fig. 2), cardiomyocytes were prepared according to the same experimental schedule as described in the electrophysiology method section. A Nikon automatic microscope (Nikon Eclipse Ti-E with a motorized stage) connected to an sCMOS camera (Andor Zyla sCMOS 4.2 MP) together with a stage-top incubator (at $37^{\circ} \mathrm{C}$ with $5 \% \mathrm{CO}_{2}$ and $20 \% \mathrm{O}_{2}$, controlled by a TOKAI HIT hypoxia gas-delivery system) were used for this experiment. A Nikon objective lens ( $\times 40$, Nikon CFI Plan Apo Lambda, NA of 0.95) was used for single-cell recordings, and the cardiomyocyte culture medium was used as the bath solution. Cells were infected with AAV9-GCaMP6f (UPenn Vector Core-Addgene) within a week of when $\mathrm{Ca}^{2+}$ imaging was performed. For testing dextromethorphan, a basal recording was acquired from each cell before treatment with dextromethorphan, and, after the basal recording, the solution was changed to cardiomyocyte culture medium with $5 \mu \mathrm{M}$ dextromethorphan or water. Recordings were then measured every $30 \mathrm{~min}$ for $2 \mathrm{~h}$ after treatment with dextromethorphan from the same cells. The $\mathrm{Ca}^{2+}$ transient frequency and the $\mathrm{Ca}^{2+}$ transient half $(50 \%)$ decay time from recordings before and after treatment were analyzed. The values of each parameter after treatment were normalized to baseline values acquired before treatment for data presentation in the corresponding figures.

In vitro CDK5 activity assay. To examine endogenous CDK5 activity, a CDK5 activity assay was conducted following the protocol that we described previously ${ }^{6}$. Human iPSC-derived cardiomyocytes differentiated from iPSCs of patients with TS were collected on day 26 or day 27 for the assay. For compound-treated groups, cardiomyocytes were treated with PRE- 084 at a dose of $5 \mu \mathrm{M}$ for $2 \mathrm{~h}$ before being collected for the assay. Cardiomyocytes were isolated from a monolayer culture and lysed with cell lysis buffer containing 1\% NP-40, $20 \mathrm{mM}$ Tris- $\mathrm{HCl}$, $137 \mathrm{mM} \mathrm{NaCl}, 1 \times$ protease inhibitor cocktail, $1 \times$ phosphatase inhibitor cocktail 3 (Sigma-Aldrich, P0044) and $1 \times$ phosphatase inhibitor cocktail (Sigma-Aldrich, P8340), pH 7.4. Protein concentrations in the samples were measured using a standard bicinchoninic acid (BCA) assay kit (Pierce-Thermo Fisher Scientific). Protein $(40 \mu \mathrm{g})$ from each sample was aliquoted and used as one sample for CDK5 immunoprecipitation. The sample was incubated with anti-CDK5 antibodyconjugated agarose beads (CDK5 (J-3) AC, Santa Cruz Biotechnology, sc-6247 AC) for $2 \mathrm{~h}$ at $4{ }^{\circ} \mathrm{C}$ (rocking continuously) for CDK5 immunoprecipitation. Resuspended bead solution $(5 \mu \mathrm{l})$ was used to immunoprecipitate CDK5 from each sample. After immunoprecipitation, beads were washed three times with cold Tris-buffered saline (TBS) and twice with room temperature TBS. A reaction mix containing $1 \times$ reaction buffer $\mathrm{A}, 50 \mu \mathrm{M}$ DTT, $50 \mu \mathrm{M}$ ATP and $1 \mu \mathrm{g}$ histone $\mathrm{H} 1$ in distilled water was added to each sample to detect CDK5 activity. The stock of PHA-793887 (Selleckchem) was diluted with DMSO and added to the corresponding samples in the PHA-793887-treated TS groups at a concentration of $5 \mu \mathrm{M}$. The same volume of DMSO was added to the remainder of the samples to achieve the same concentration of DMSO in all reactions. A series of samples for a standard curve were prepared based on the manufacturer's instructions to determine ATP-ADP conversion from the luminescence signals in every round of the experiment. Kinase reaction tubes with the reaction mixes were incubated at $26-27^{\circ} \mathrm{C}$ for $60 \mathrm{~min}$ for the kinase reaction. The ADP-Glo reagent (Promega) was then added to the reactions, which were incubated for $40 \mathrm{~min}$ at $26-27^{\circ} \mathrm{C}$ to deplete ATP in the reactions. Next, the detection reagent was added to the reactions, which were incubated for $45 \mathrm{~min}$ at $26-27^{\circ} \mathrm{C}$. A $20-\mu \mathrm{l}$ sample from each tube was then transferred into a 96-well microplate, and luminescence was measured with the GloMax 96 Microplate Luminometer (1.9.2, Promega) with an integration time of $1.5 \mathrm{~s}$. Luminescence values were converted into ATP-ADP conversion values based on the standard curve. Four rounds of the experiment were conducted. Values from each round of experiments were normalized to the average value of the cardiomyocyte samples from patients with TS without PHA-793887 before being pooled together for the final analysis that is shown in Extended Data Fig. 1b.

Western blotting of human cardiomyocytes. Anti-p35 (CDK5R1, rabbit polyclonal antibody, sc-820 'discontinued', clone C-19, 1:1,000 dilution, Santa Cruz; rabbit recombinant monoclonal antibody, C64B10, 1:500 dilution, Cell Signaling Technology), anti-CDK5 (mouse monoclonal antibody, MABS50, clone 1H3 'discontinued', 1:1,000 dilution, Millipore; rabbit recombinant monoclonal antibody, ab40773, 1:2,000 dilution, Abcam), anti-SIGMAR1 (rabbit polyclonal antibody, 42-3300, 1:1,000 dilution, Thermo Fisher Scientific), anti-ATF4 (rabbit recombinant monoclonal antibody, ab184909, 1:500 dilution, Abcam), antiGAPDH (rabbit recombinant monoclonal antibody, ab181602, 1:10,000 dilution, Abcam) and anti- $\beta$-tubulin (mouse monoclonal antibody, T5201, clone TUB 2.1, 1:8,000 dilution, Sigma-Aldrich) antibodies were used for western blotting. Some cardiomyocytes from patients with TS were treated with $5 \mu \mathrm{M}$ PRE-084 in cardiomyocyte culture medium for $2 \mathrm{~h}$ before being collected for western blotting. The cardiomyocytes from patients with TS from the same round of differentiation were collected as a control. Cells were isolated from the monolayer and lysed with cell lysis buffer containing $1 \%$ Triton X-100, $50 \mathrm{mM}$ Tris- $\mathrm{HCl}, 150 \mathrm{mM} \mathrm{NaCl}$, $250 \mathrm{mM}$ sucrose, $1 \times$ protease inhibitor cocktail, $1 \times$ phosphatase inhibitor cocktail 3 and $1 \times$ phosphatase inhibitor cocktail, $\mathrm{pH}$ 7.4. The concentration of total protein in each sample was measured using the BCA assay kit. Next, $20 \mu \mathrm{g}$ protein from each sample was aliquoted and denatured with sample buffer containing urea and boiled for $5 \mathrm{~min}$ at $95^{\circ} \mathrm{C}$. Samples were loaded onto Tris-HCl-based SDS-PAGE gels with a $5 \%$ stacking gel and a $10 \%$ separation gel along with the ladder (pre-stained SDS-PAGE standards, broad range, 161-0318, Bio-Rad). Proteins were electrotransferred to PVDF membranes (Invitrolon PVDF, LC2005, NovexThermo Fisher Scientific) using the XCell SureLock Mini-Cell system (Thermo Fisher Scientific) overnight at $4^{\circ} \mathrm{C}$. The next day, membranes were blocked with SuperBlock Blocking Buffer in PBS (Thermo Fisher Scientific, 27515) for $30 \mathrm{~min}$ at room temperature and then incubated with primary antibody (diluted in the SuperBlock Blocking Buffer) overnight, followed by incubation with the corresponding secondary antibody (Pierce-Thermo Fisher Scientific, anti-mouse, 31430; anti-rabbit, 31460, 1:8,000 dilution in SuperBlock Blocking Buffer) for $30 \mathrm{~min}$ at room temperature. Membranes were then incubated with the Pierce ECL Western blotting substrate (Thermo Fisher Scientific, 32209) followed by exposure to X-ray films (CL-XPosure film, Thermo Fisher Scientific, 34091) in a dark room. For sequential immunoblotting, membranes were stripped with stripping buffer containing $62.5 \mathrm{mM}$ Tris-HCl, $2 \%$ SDS and $114 \mathrm{mM} 2$-mercaptoethanol at $42^{\circ} \mathrm{C}$ for $15-20 \mathrm{~min}$ and washed six times with PBS. The stripped membranes were then reblocked with SuperBlock Blocking Buffer for the next immunoblot. For immunoblotting for $\beta$-tubulin, the membrane was stripped, reblocked with the SuperBlock Blocking Buffer and incubated with anti- $\beta$-tubulin antibody at room temperature for $30 \mathrm{~min}$. The remaining steps were the same. The western blot analysis to examine 35 protein expression in cardiomyocytes from patients with TS before and after treatment with $5 \mu \mathrm{M}$ PRE- 084 was repeated six times with independent samples from different rounds of differentiation for validation. The western blot analysis to examine CDK5 expression in cardiomyocytes from patients with TS before and after treatment with $5 \mu \mathrm{M}$ PRE-084 was repeated four times using samples from different rounds of differentiation for validation. Western blot band intensities were quantified using ImageJ, and values were normalized to the corresponding $\beta$-tubulin band intensities or GAPDH band intensities. Values from cardiomyocytes from patients with TS $2 \mathrm{~h}$ after treatment with $5 \mu \mathrm{M}$ PRE-084 were normalized to values from cardiomyocytes from patients with TS without treatment for presentation in the corresponding figures. Values from cardiomyocytes from patients with LQTS1, LQTS2 or TS were normalized to values from control cardiomyocytes for presentation in the corresponding figures. Blotting images (uncropped, with molecular markers) are also shown in Supplementary Fig. 1.

Immunocytochemistry. The PLA was conducted using the Sigma-Aldrich Duolink kit (DUO92101 or DUO92103). Human iPSC-derived cardiomyocytes were treated with $5 \mu \mathrm{M}$ dextromethorphan for $2 \mathrm{~h}$ at $37^{\circ} \mathrm{C}$ and then washed with PBS. Next, samples were fixed in a solution of $4 \%$ PFA and $2 \%$ sucrose for $15 \mathrm{~min}$ at room temperature, washed three times with PBS and permeabilized for $10 \mathrm{~min}$ at room temperature with $0.2 \%$ Triton X-100. Permeabilized samples were then washed three times with PBS and blocked for $1 \mathrm{~h}$ at $37^{\circ} \mathrm{C}$ in a humidity chamber using the blocking solution from the Duolink kit. Samples were next incubated with primary antibody overnight at $4{ }^{\circ} \mathrm{C}\left(K C N Q 1\right.$, anti- $\mathrm{K}_{\mathrm{V}} 7.1$, Alomone Labs, APC 022, 1:500; anti-SIGMAR1, Santa Cruz, sc-137075 (mouse), 1:500; anti-SIGMAR1, Abcam, ab53852 (rabbit), 1:500; KCNH2, anti-hERG, Santa Cruz, sc-377388, 1:500 anti-CDK5, Abcam, ab40773, 1:500; anti-Ca 1.2 , Alomone Labs, ACC-003, 1:100). The remainder of the procedure was performed following the Sigma-Aldrich PLA fluorescence-detection protocol. Cell fluorescent images were acquired using a confocal microscope (Nikon, A1RMP) and epifluorescent microscopes, which were used for the above physiological recordings. PLA signal quantifications were performed blindly: M.Y. coded image files, and R.B. and A.D.K. analyzed signals using ImageJ-win64 (Fiji) in a blinded manner. For immunostaining, cells were fixed and stained using WGA (conjugated with Alexa Fluor 647, Thermo Fisher Scientific, W34266, $5 \mu \mathrm{g} \mathrm{ml}^{-1}$ ). Next, cells were permeabilized and stained with anti-hERG (Alomone Labs, APC-062, 1:200) or anti-K 7.1 (Alomone Labs, APC-022, 1:200) antibodies followed by the corresponding secondary antibody and mounted with mounting medium containing DAPI (from the above Duolink kit, Sigma-Aldrich). Quantifications of WGA-hERG or WGA-K 7.1 colocalization were conducted blindly: R.B. coded image files, and L.S. analyzed images using ImageJ-win64 (Fiji) and quantified the fraction of green over red signals in a blinded manner. R.B. analyzed values using GraphPad Prism (version 7.0).

Quantitative RT-PCR. RNA from control cardiomyocytes, cardiomyocytes from patients with TS without treatment and $2 \mathrm{~h}$ after treatment with $5 \mu \mathrm{M}$ PRE-084 was prepared using the RNeasy Mini kit and the RNase-Free DNase set (Qiagen). Mouse heart tissue RNA samples were also prepared using the same method. cDNA was synthesized using the SuperScript III First-Strand Synthesis System for RT-PCR (Thermo Fisher Scientific). cDNA $(21 \mu \mathrm{l})$ was diluted with 
DNase-free water (Thermo Fisher Scientific) at a ratio of $1: 1$, and $1 \mu$ of the samples was used for quantitative RT-PCR (qPCR) analysis. SYBR Advantage qPCR Premix (Clontech-Takara Bio) and StepOnePlus Real-Time PCR systems (Thermo Fisher Scientific) with StepOne software (version 2.3, Life Technologies) were used for qPCR. Primer sets for detecting the $h C D K 5, h S I G M A R 1, m K c n q 1, h G A P D H$ and $m$ Gapdh transcripts (h, human; $m$, mouse) were as follows: hCDK5 (forward, 5'-GGCTTCAGGTCCCTGTGTAG-3'; reverse, 5' -ATGGTGACCTCGATCCTG AG-3'), hSIGMAR1 (forward, 5'-GGGAGACGGTAGTACACGG-3'; reverse, 5'-AGGAGCGAAGAGTATAGAAGAGG-3'), hGAPDH (forward, 5'-GATGAC ATCAAGAAGGTGGTGA-3'; reverse, 5' -GTCTACATGGCAACTGTGAGGA-3') mKcnq1 (forward, 5' -CTTGTGGTGTTCTTTGGGACA-3'; reverse, 5'CCCAGA TGCCCACGTACTT-3'), mGapdh (forward, $5^{\prime}$-CTTCACCACCATGGAGAA GG-3'; reverse, $5^{\prime}$-TGAAGTCGCAGGAGACAACC-3'). Two independent iPSC lines from patients with TS (TS1-E3-5, $n=6$; TS2-E7-1, $n=3$ ) and two paired isogenic control lines (TS1-E3-5c3-14, $n=3$; TS2-E7-1c6, $n=3$; ref. ${ }^{6}$ ) as well as two independent normal participant-derived iPSC lines (IM-E1-5, $n=3$; NH-E1-1, $n=3$ ) were used for quantitative RT-PCR (Extended Data Fig. 2i). Cardiomyocytes derived from iPSCs of patients with TS (TS1-E3-5, $n=4$ independent differentiations) with and without dextromethorphan treatment were used with hGAPDH (version 2, forward, 5'-CTCACCGGATGCACCAATGTT-3'; reverse, 5' -CGCG TTGCTCACAATGTTCAT-3'), hKCNQ1 (isoform 1, forward, 5'-GCGTCTCC ATCTACAGCACG-3'; reverse, 5'-GAAGTGGTAAACGAAGCATTTCC-3'), hKCNQ1 (isoform 2, forward, 5'-GACTTCCTCATCGTCCTGGT-3'; reverse, $5^{\prime}$-GAAGAACACCACCAGCACGA- $3^{\prime}$ ) and hERG (KCNH2; forward, 5' -TTCG ACCTGCTCATCTTCGG-3'; reverse, 5' -CGATGCGTGAGTCCATGTGT-3') (Extended Data Fig. 5l,m). Following the manufacturer's instructions, human normal cardiomyocytes (Fujifilm, iCell, 11713) were transfected using ViaFect (Promega) and the hATF4 plasmid that was made from LV-SD (CAG promoter, Addgene, 12105, LV-Cre-SD, no longer available currently; Cre was removed by using EcoRI and XhoI sites). The human ATF4 DNA fragment was synthesized (IDT, gBlock) and subcloned into the LV-SD vector. High transfection efficiency $(\sim 70 \%)$ in human cardiomyocytes was confirmed using a yellow fluorescent protein (YFP) plasmid. hATF4 (forward, 5' -CCCTTCACCTTCTTACAACCTC-3'; reverse, $5^{\prime}$-TGCCCAGCTCTAAACTAAAGGA-3') and hSIGMAR1 (version 2, forward, 5' -CGAAGAGATAGCGCAGTTGG-3'; reverse, 5' -TCCACGATCAGA CGAGAGAAG- $3^{\prime}$ ) were used with the above hGAPDH primer set (version 2) for ATF4-transfected cardiomyocytes (Extended Data Fig. 2o,p). Transfected cells were also used for western blotting using anti-hSIGMAR1 antibody (Extended Data Fig. 2q,r). The above primer sets were synthesized by Eton Bioscience or IDT. The $C_{\mathrm{t}}$ value of each sample at $50 \%$ of the amplification curve was used, and GAPDH or Gapdh values were used to normalize the expression of target molecules.

Mouse electrocardiography. The conditional mouse model of TS was generated by M.Y., targeting the Rosa26 locus with a mutation corresponding to G406R in the $\mathrm{Ca}^{2+}$ channel Cacnalc gene ${ }^{37}$ (Fig. 5a). $\alpha$-MHC ${ }^{\text {MerCreMer }}$ transgenic mice $\left(M y h 6^{\mathrm{Cre} / \mathrm{Esr} 1}\right.$, JAX 5657) were used for this mouse study. When $\alpha-M C^{\text {MerCreMer }}$ transgenic mice are bred with mice from the TS model containing loxP-flanked sequences, tamoxifen-inducible Cre-mediated recombination is expected to result in deletion of the floxed sequences in heart cells of the offspring to specifically overexpress TS mutant channels in the heart. Mice were maintained using a standard diet under a 12-h light-dark cycle. Subcutaneous 4-lead electrocardiograms of isofluraneanesthetized adult mice (backcrossed with C57BL/6 mice, Envigo, 044, 12-weekold females) were performed using emka ECG and recorded using iox 2.8.0.19 (emka TECHNOLOGIES). Recordings were conducted on days 4 and 11 after tamoxifen (Sigma-Aldrich, $50 \mu \mathrm{g}$ per body weight $(\mathrm{g})$ per $\mathrm{d}$ per mouse, that is, approximately $1 \mathrm{mg}$ per d per mouse) was administrated on days $1-3$. Dextromethorphan-quinidine (Sigma-Aldrich, Q0875; dextromethorphan: quinidine ratio, 2:1) was dissolved in drinking water for dosing. RR and QT intervals were measured manually using ecgAUTO version 2.8.1.27 (emka TECHNOLOGIES), Microsoft Excel for Mac 2011 (Microsoft) and Prism 6 or Prism 7 software (GraphPad). To evaluate the pharmacokinetic profile of dextromethorphan with the designed dosing strategy in mice, we conducted a pharmacokinetic analysis. Twelve-week-old C57BL/6 female mice were used for the analysis and were dosed in the same manner as for the ECG experiments. Blood was sampled from the dosed animals at $8 \mathrm{AM}$, noon and $8 \mathrm{PM}$ on days 4 and 11 after treatment and collected through the tail vein using a heparinized microhematocrit capillary tube (Fisher Scientific, 22-362566). Plasma was isolated by centrifugation and transferred to a $1.5-\mathrm{ml}$ clear Eppendorf tube (Eppendorf, 22364120) containing $5 \mu \mathrm{l} \mathrm{0.5} \mathrm{M} \mathrm{EDTA} \mathrm{(Thermo} \mathrm{Fisher} \mathrm{Scientific,} \mathrm{15575020)}$ and stored at $-80^{\circ} \mathrm{C}$ until further analysis. The plasma concentration of dextromethorphan from dosed animals was measured and analyzed at the Columbia University Biomarkers Core Laboratory using a validated customized liquid chromatography mass spectrometry (Agilent 6410A mass spectrometer coupled with an Agilent 1290 Infinity UHPLC)-based assay.

Global quantitative proteomics by mass spectrometry. For global quantitative proteomics of mouse ventricular heart samples (from the above mouse at day 11), tandem mass tag (TMT)-based quantitative proteomics was used. In brief, a frozen mouse ventricular heart was lysed by bead beating in $9 \mathrm{M}$ urea and $200 \mathrm{mM}$
EPPS (pH 8.5) supplemented with protease and phosphatase inhibitors. Samples were reduced with $5 \mathrm{mM}$ TCEP and alkylated with $10 \mathrm{mM}$ iodoacetamide, which was quenched with $10 \mathrm{mM}$ DTT. A total of $400 \mu \mathrm{g}$ protein was precipitated by the chloroform-methanol method. Protein was reconstituted in $200 \mathrm{mM}$ EPPS ( $\mathrm{pH}$ 8.5) and digested with Lys-C overnight and trypsin for $6 \mathrm{~h}$, both at a proteaseto-peptide ratio of 1:50. Digested peptides were quantified using a NanoDrop at $280 \mathrm{~nm}$, and $200 \mu \mathrm{g}$ peptide from each sample was labeled with $800 \mu \mathrm{g}$ TMT reagent using an 11-plex TMT kit ${ }^{57}$. TMT labels were checked, $0.5 \mu \mathrm{g}$ of each sample was pooled, desalted and analyzed by the short synchronous precursor-selection (SPS)-MS 3 method, and, using the normalization factor, samples were bulk mixed at 1:1 across all channels, and bulk mixed samples were fractionated using the Pierce High pH Reversed-Phase Peptide Fractionation kit (Thermo Fisher Scientific), and each fraction was dried down in a SpeedVac. Dried peptides were dissolved in $10 \mu \mathrm{l} 3 \%$ acetonitrile, $0.1 \%$ formic acid and injected using SPS-MS 3 . The UltiMate 3000 RSLCnano system (Thermo Scientific) and the EASY-Spray source (Thermo Fisher Scientific) with the Acclaim PepMap100 2-cm $\times 75-\mu \mathrm{m}$ trap column (Thermo Fisher Scientific) and the EASY-Spray PepMap RSLC C18 $(50-\mathrm{cm} \times 75-\mu \mathrm{m}$ ID) column (Thermo Fisher Scientific) were used to separate fractioned peptides with a $5-30 \%$ acetonitrile gradient in $0.1 \%$ formic acid over $127 \mathrm{~min}$ at a flow rate of $250 \mathrm{nl} \mathrm{min}^{-1}$. After each gradient, the column was washed with $90 \%$ buffer B for 5 min and re-equilibrated with $98 \%$ buffer A $(0.1 \%$ formic acid, 100\% HPLC-grade water) for $40 \mathrm{~min}$. For BPRP-separated proteome fractions, full MS spectra were acquired with the Orbitrap Fusion Tribrid Mass Spectrometer (Thermo Fisher Scientific) at a resolution of 120,000. The ten most intense $\mathrm{MS}^{1}$ ions were selected for $\mathrm{MS}^{2}$ analysis. The isolation width was set at $0.7 \mathrm{Da}$, and isolated precursors were fragmented by CID at a normalized collision energy of $35 \%$ and analyzed in the ion trap using 'turbo' scan speed. Following acquisition of each $\mathrm{MS}^{2}$ spectrum, an SPS-MS ${ }^{3}$ scan was collected on the top ten most intense ions in the MS ${ }^{2}$ spectrum. SPS-MS ${ }^{3}$ precursors were fragmented by higher-energy collision-induced dissociation at a normalized collision energy of $65 \%$ and analyzed using the Orbitrap. Raw mass spectrometric data were analyzed using Proteome Discoverer 2.2 to perform the database search and TMT reporter ion quantification. TMT tags on lysine residues and peptide $\mathrm{N}$ termini $(+229.163 \mathrm{Da})$ and carbamidomethylation of cysteine residues $(+57.021 \mathrm{Da})$ were set as static modifications, while oxidation of methionine residues $(+15.995 \mathrm{Da})$, deamidation $(+0.984 \mathrm{Da})$ on asparagine and glutamine and phosphorylation $(+79.966 \mathrm{Da})$ on serine, threonine and tyrosine were set as variable modifications. Data were searched against UniProt data from a mouse with peptide-spectrum matches and protein level at $1 \%$ FDR. Signal-to-noise measurements of each protein were normalized so that the sum of the signal for all proteins in each channel was equivalent to account for equal protein loading. Results obtained from Proteome Discoverer 2.2 were further analyzed using the Perseus statistical package $^{58}$, which is part of the MaxQuant distribution. Significantly changed protein abundance was determined by ANOVA with $P<0.05$ (permutation-based FDR correction). Pathway analysis was performed using ingenuity IPA.

Western blotting for potassium channels. For western blot analysis of mouse tissue samples, remaining mouse tissue from the proteomic analysis was used and further resuspended in a solution containing $9 \mathrm{M}$ urea, $50 \mathrm{mM}$ ammonium bicarbonate and protease and phosphatase inhibitors. Protein concentrations were determined using a standard BCA assay. Protein $(30 \mu \mathrm{g})$ from each sample was aliquoted and denatured in a urea sample buffer and boiled for $5 \mathrm{~min}$ at $95^{\circ} \mathrm{C}$. Samples were loaded onto Tris-HCl-based SDS-PAGE gels for electrophoresis and electrotransferred to PVDF membranes using the XCell SureLock MiniCell system overnight. The next day, samples were placed in blocking solution ( $5 \%$ skim milk in TBS) overnight at $4^{\circ} \mathrm{C}$. The following day, membranes were incubated with primary antibody solution overnight at $4{ }^{\circ} \mathrm{C}$ (anti- $\mathrm{K}_{\mathrm{V}} 7.1$ antibody (KCNQ1), Alomone Labs, APC-022, 1:2,000 dilution; anti-GAPDH antibody, Abcam, ab181602, 1:10,000 dilution; anti- $\beta$-tubulin antibody, Sigma-Aldrich, T5201, 1:8,000 dilution). Membranes were then incubated with the corresponding secondary antibody for $1 \mathrm{~h}$ at room temperature and then developed with Pierce ECL Western Blotting Substrate, followed by exposure to X-ray films in a dark room. Blotting images (uncropped, with molecular markers) are also shown in Supplementary Fig. 1. For western blotting for $\mathrm{K}^{+}$channels in human iPSC-derived cardiomyocytes, some of the cardiomyocytes from patients with TS were treated with $5 \mu \mathrm{M}$ dextromethorphan in cardiomyocyte culture medium for $2 \mathrm{~h}$ before being collected for western blotting. The cardiomyocytes from patients with TS from the same round of differentiation were collected as a control. Anti-hERG (KCNH2) antibody (Santa Cruz, F-12, sc-377388, 1:500 dilution) and the same anti-K 7.1 (KCNQ1) antibody were used for the samples (Extended Data Fig. 5d,k). Cells were lysed in $1 \times$ cell lysis buffer (Cell Signaling Technology, 9083) containing $1 \times$ protease inhibitor cocktail and $1 \times$ phosphatase inhibitor cocktail 3 (SigmaAldrich, P0044) with $1 \times$ phosphatase inhibitor cocktail (Sigma-Aldrich, P8340). After this, samples were processed and analyzed using the same procedure as that for mouse tissues.

Statistics and reproducibility. Statistics used for every figure are indicated in the corresponding figure legends. Student's $t$-test (paired and unpaired) was conducted with the $t$-test function in Microsoft Excel software. Student's $t$-tests 
were two-tailed. One-way ANOVA with Tukey's, Sidak's, Bonferroni's or Dunnett's post hoc multiple-comparison analysis was conducted with GraphPad Prism 6 or 7 software. All data meet the assumptions of the statistical tests. All samples used in this study were biological repeats, not technical repeats. All experiments were conducted using at least two independent experimental materials or cohorts to reproduce similar results. No samples were excluded from analysis in this study, except one outlier (defined by Grubbs' test) in the analysis for plasma dextromethorphan concentrations (Extended Data Fig. 8a). All graphs in figures, except the plasma concentration of dextromethorphan in Extended Data Fig. 8a, show mean \pm s.d.. Extended Data Fig. 8 a shows mean \pm s.e.m.

Reporting Summary. Further information on research design is available in the Nature Research Reporting Summary linked to this article.

\section{Data availability}

Source data are provided with this paper.

Received: 27 October 2021; Accepted: 22 December 2021; Published online: 17 February 2022

\section{References}

1. Berridge, M. J., Bootman, M. D. \& Roderick, H. L. Calcium signalling: dynamics, homeostasis and remodelling. Nat. Rev. Mol. Cell Biol. 4, 517-529 (2003).

2. Wagner, S., Maier, L. S. \& Bers, D. M. Role of sodium and calcium dysregulation in tachyarrhythmias in sudden cardiac death. Circ. Res. 116, 1956-1970 (2015)

3. Splawski, I. et al. $\mathrm{Ca}_{\mathrm{V}} 1.2$ calcium channel dysfunction causes a multisystem disorder including arrhythmia and autism. Cell 119, 19-31 (2004).

4. Mahida, S. et al. Genetics of congenital and drug-induced long QT syndromes: current evidence and future research perspectives. J. Interv. Card. Electrophysiol. 37, 9-19 (2013).

5. Yazawa, M. et al. Using induced pluripotent stem cells to investigate cardiac phenotypes in Timothy syndrome. Nature 471, 230-234 (2011).

6. Song, L., Park, S. E., Isseroff, Y., Morikawa, K. \& Yazawa, M. Inhibition of CDK5 alleviates the cardiac phenotypes in Timothy syndrome. Stem Cell Rep. 9, 50-57 (2017).

7. Massard, C. et al. A first in man, phase I dose-escalation study of PHA-793887, an inhibitor of multiple cyclin-dependent kinases (CDK2, 1 and 4) reveals unexpected hepatotoxicity in patients with solid tumors. Cell Cycle 10, 963-970 (2011).

8. Malumbres, M. Cyclin-dependent kinases. Genome Biol. 15, 122 (2014)

9. Maurice, T. \& Goguadze, N. Sigma-1 $(\sigma 1)$ receptor in memory and neurodegenerative diseases. Handb. Exp. Pharmacol. 244, 81-108 (2017).

10. Kim, F. J. \& Maher, C. M. Sigma 1 pharmacology in the context of cancer. Handb. Exp. Pharmacol. 244, 237-308 (2017).

11. Ruscher, K. \& Wieloch, T. The involvement of the sigma-1 receptor in neurodegeneration and neurorestoration. J. Pharmacol. Sci. 127, 30-35 (2015).

12. Morales-Lazaro, S. L., Gonzalez-Ramirez, R. \& Rosenbaum, T. Molecular interplay between the sigma-1 receptor, steroids, and ion channels. Front. Pharmacol. 10, 419 (2019).

13. Tsai, S. Y., Pokrass, M. J., Klauer, N. R., Nohara, H. \& Su, T. P. Sigma-1 receptor regulates tau phosphorylation and axon extension by shaping p35 turnover via myristic acid. Proc. Natl Acad. Sci. USA 112, $6742-6747$ (2015).

14. Schmidt, H. R. et al. Crystal structure of the human $\sigma 1$ receptor. Nature 532, 527-530 (2016).

15. Su, T. P. et al. Sigma compounds derived from phencyclidine: identification of PRE-084, a new, selective sigma ligand. J. Pharmacol. Exp. Ther. 259, 543-550 (1991).

16. Figgitt, D. P. \& McClellan, K. J. Fluvoxamine. An updated review of its use in the management of adults with anxiety disorders. Drugs 60, 925-954 (2000).

17. Nguyen, L., Robson, M. J., Healy, J. R., Scandinaro, A. L. \& Matsumoto, R. R Involvement of sigma-1 receptors in the antidepressant-like effects of dextromethorphan. PLoS ONE 9, e89985 (2014).

18. De Blasio, F. et al. Cough management: a practical approach. Cough 7, 7 (2011).

19. Taylor, C. P., Traynelis, S. F., Siffert, J., Pope, L. E. \& Matsumoto, R. R. Pharmacology of dextromethorphan: relevance to dextromethorphan/ quinidine (Nuedexta ${ }^{\varpi}$ ) clinical use. Pharmacol. Ther. 164, 170-182 (2016).

20. Rosen, H. Dextromethorphan/quinidine sulfate for pseudobulbar affect. Drugs Today 44, 661-668 (2008).

21. Chaki, S., Tanaka, M., Muramatsu, M. \& Otomo, S. NE-100, a novel potent sigma ligand, preferentially binds to sigma 1 binding sites in guinea pig brain. Eur. J. Pharmacol. 251, R1-R2 (1994).

22. Okuyama, S. et al. NE-100, a novel sigma receptor ligand: in vivo tests. Life Sci. 53, PL285-PL290 (1993).

23. Tanaka, M., Shirasaki, T., Kaku, S., Muramatsu, M. \& Otomo, S. Characteristics of binding of $[3 \mathrm{H}] \mathrm{NE}-100$, a novel sigma-receptor ligand, to guinea-pig brain membranes. Naunyn Schmiedebergs Arch. Pharmacol. 351, 244-251 (1995).
24. Mitsuda, T. et al. Sigma-1Rs are upregulated via PERK/eIF2 $\alpha / A T F 4$ pathway and execute protective function in ER stress. Biochem. Biophys. Res. Commun. 415, 519-525 (2011).

25. Bhuiyan, M. S., Tagashira, H. \& Fukunaga, K. Sigma-1 receptor stimulation with fluvoxamine activates Akt-eNOS signaling in the thoracic aorta of ovariectomized rats with abdominal aortic banding. Eur. J. Pharmacol. 650, 621-628 (2011).

26. Montastruc, G. et al. Drugs and dilated cardiomyopathies: a case/noncase study in the French PharmacoVigilance Database. Br. J. Clin. Pharmacol. 69, 287-294 (2010).

27. Hashimoto, K. Sigma-1 receptors and selective serotonin reuptake inhibitors: clinical implications of their relationship. Cent. Nerv. Syst. Agents Med. Chem. 9, 197-204 (2009)

28. Chen, T. W. et al. Ultrasensitive fluorescent proteins for imaging neuronal activity. Nature 499, 295-300 (2013).

29. Mueller, B. H. 2nd et al. Sigma-1 receptor stimulation attenuates calcium influx through activated L-type voltage gated calcium channels in purified retinal ganglion cells. Exp. Eye Res. 107, 21-31 (2013).

30. Pan, B., Guo, Y., Kwok, W. M., Hogan, Q. \& Wu, H. E. Sigma-1 receptor antagonism restores injury-induced decrease of voltage-gated $\mathrm{Ca}^{2+}$ current in sensory neurons. J. Pharmacol. Exp. Ther. 350, 290-300 (2014).

31. Tchedre, K. T. et al. Sigma-1 receptor regulation of voltage-gated calcium channels involves a direct interaction. Invest. Ophthalmol. Vis. Sci. 49, 4993-5002 (2008)

32. Crottes, D. et al. Sig1R protein regulates hERG channel expression through a post-translational mechanism in leukemic cells. J. Biol. Chem. 286, 27947-27958 (2011)

33. Balasuriya, D. et al. A direct interaction between the sigma-1 receptor and the hERG voltage-gated $\mathrm{K}^{+}$channel revealed by atomic force microscopy and homogeneous time-resolved fluorescence $\left(\mathrm{HTRF}^{\varpi}\right)$. J. Biol. Chem. 289, 32353-32363 (2014).

34. Wu, Z. Y., Yu, D. J., Soong, T. W., Dawe, G. S. \& Bian, J. S. Progesterone impairs human ether-a-go-go-related gene (HERG) trafficking by disruption of intracellular cholesterol homeostasis. J. Biol. Chem. 286, 22186-22194 (2011).

35. Liang, P. et al. Drug screening using a library of human induced pluripotent stem cell-derived cardiomyocytes reveals disease-specific patterns of cardiotoxicity. Circulation 127, 1677-1691 (2013).

36. Mehta, A. et al. Re-trafficking of hERG reverses long QT syndrome 2 phenotype in human iPS-derived cardiomyocytes. Cardiovasc. Res. 102, 497-506 (2014)

37. Pasca, S. P. et al. Using iPSC-derived neurons to uncover cellular phenotypes associated with Timothy syndrome. Nat. Med. 17, 1657-1662 (2011).

38. Salama, G. \& London, B. Mouse models of long QT syndrome. J. Physiol. 578, 43-53 (2007).

39. Landry, N. M., Cohen, S. \& Dixon, I. M. C. Periostin in cardiovascular disease and development: a tale of two distinct roles. Basic Res. Cardiol. 113, 1 (2018).

40. McCalmon, S. A. et al. Modulation of angiotensin II-mediated cardiac remodeling by the MEF2A target gene Xirp2. Circ. Res. 106, 952-960 (2010).

41. Yazawa, $\mathrm{M}$. et al. TRIC channels are essential for $\mathrm{Ca}^{2+}$ handling in intracellular stores. Nature 448, 78-82 (2007).

42. Brandt, R. R., Wright, R. S., Redfield, M. M. \& Burnett, J. C. Jr. Atrial natriuretic peptide in heart failure. J. Am. Coll. Cardiol. 22, 86A-92A (1993).

43. Thiel, W. H. et al. Proarrhythmic defects in Timothy syndrome require calmodulin kinase II. Circulation 118, 2225-2234 (2008).

44. Ottolia, M., Torres, N., Bridge, J. H., Philipson, K. D. \& Goldhaber, J. I. $\mathrm{Na} / \mathrm{Ca}$ exchange and contraction of the heart. J. Mol. Cell. Cardiol. 61, 28-33 (2013).

45. Deisemann, H. et al. Effects of common antitussive drugs on the hERG potassium channel current. J. Cardiovasc. Pharmacol. 52, 494-499 (2008).

46. Itzhaki, I. et al. Modelling the long QT syndrome with induced pluripotent stem cells. Nature 471, 225-229 (2011).

47. Uresin, Y., Ozek, M. \& Sevgi, S. Protective effects of dextromethorphan and tizanidine on ouabain-induced arrhythmias. Methods Find. Exp. Clin. Pharmacol. 24, 421-423 (2002).

48. Boland, D. M., Rein, J., Lew, E. O. \& Hearn, W. L. Fatal cold medication intoxication in an infant. J. Anal. Toxicol. 27, 523-526 (2003).

49. Kaplan, B., Buchanan, J. \& Krantz, M. J. QTc prolongation due to dextromethorphan. Int. J. Cardiol. 148, 363-364 (2011).

50. Nitert, M. D., Nagorny, C. L., Wendt, A., Eliasson, L. \& Mulder, H. Ca 1.2 rather than $\mathrm{Ca}_{\mathrm{V}} 1.3$ is coupled to glucose-stimulated insulin secretion in INS-1 832/13 cells. J. Mol. Endocrinol. 41, 1-11 (2008).

51. Stracina, T. \& Novakova, M. Cardiac sigma receptors-an update. Physiol. Res. 67, S561-S576 (2018).

52. Lewis, R., Li, J., McCormick, P. J., C, L. H. H. \& Jeevaratnam, K. Is the sigma-1 receptor a potential pharmacological target for cardiac pathologies? A systematic review. Int. J. Cardiol. Heart Vasc. 26, 100449 (2020).

53. Reis, G. et al. Effect of early treatment with fluvoxamine on risk of emergency care and hospitalisation among patients with COVID-19: the TOGETHER randomised, platform clinical trial. Lancet Glob. Health 10, e42-e51 (2021). 
54. Gordon, D. E. et al. A SARS-CoV-2 protein interaction map reveals targets for drug repurposing. Nature 583, 459-468 (2020).

55. Song, L. et al. Dual optical recordings for action potentials and calcium handling in induced pluripotent stem cell models of cardiac arrhythmias using genetically encoded fluorescent indicators. Stem Cells Transl. Med. 4, 468-475 (2015).

56. Skarnes, W. C., Pellegrino, E. \& McDonough, J. A. Improving homologydirected repair efficiency in human stem cells. Methods 164-165, 18-28 (2019).

57. Navarrete-Perea, J., Yu, Q., Gygi, S. P. \& Paulo, J. A. Streamlined tandem mass tag (SL-TMT) protocol: an efficient strategy for quantitative (phospho)proteome profiling using tandem mass tag-synchronous precursor selection-MS ${ }^{3}$. J. Proteome Res. 17, 2226-2236 (2018).

58. Tyanova, S. et al. The Perseus computational platform for comprehensive analysis of (prote)omics data. Nat. Methods 13, 731-740 (2016).

59. Pan, L. et al. Isolation and characterization of alternatively spliced variants of the mouse sigma ${ }_{1}$ receptor gene, Sigmar1. PLoS ONE 12, e0174694 (2017).

60. Nie, X. et al. An appropriate loading control for western blot analysis in animal models of myocardial ischemic infarction. Biochem. Biophys. Rep. 12 108-113 (2017).

\section{Acknowledgements}

We thank J.C. Wu (Stanford University) for sharing iPSC lines from patients with LQTS1 or LQTS2; G. Pitt (Weill Cornell Medicine), R. Katz, A. Rinderspacher, S. Deng, B. Corneo, R. Robinson, H. Colecraft, S. Marx, R. Kass, E. Passagué and J. Stein (Columbia University) and R. Kaye (SSI Strategy) for helpful discussions; and S. Asano (Pfizer) for helpful input about image analysis. This work was supported by NIH K99/ R00HL111345, NIH R01HL138486, the Columbia University Core Usage Funding Program and a Helmsley Stem Cell Seed Grant (to M.Y.), NIH R01HL136758 (to J.P.M.), NIH F31HL142239 (to J.R.Q.), a JSPS postdoctoral fellowship (to K.M.) and the Columbia TRANSFORM TL1 training program (to R.B. and D.A.).

\section{Author contributions}

L.S. and M.Y. conceived of and designed this project. L.S., R.B., K.M., J.R.Q. and M.Y. designed experiments. L.S., R.B., K.M., J.R.Q., A.D.K., D.A., K.Y., H.E.Y., A.C., R.Y., A.P., D.S., L.C.J., T.M.L., F.K., R.K.S., J.P.M. and M.Y. performed experiments and analyzed data: M.Y. conducted whole-cell patch clamping; R.K.S. conducted proteomics; F.K. conducted computational modeling. L.S., R.B., K.M., J.R.Q., J.S.W. and M.Y. interpreted data. M.Y. wrote the manuscript. L.S., R.B., K.M., J.R.Q., D.A., T.M.L. and J.P.M. proofread and edited the manuscript. All authors approved the manuscript.

\section{Competing interests}

L.S., R.B., K.M., J.R.Q. and M.Y. (inventors) filed a patent (attorney docket no. 01001/005273-US1; status, filed, 4 October 2019) related to this manuscript. This patent is for using SIGMAR1 agonists to treat LQTS (types 1,2 and 8) and related cardiac channelopathy. The remaining authors declare no competing interests.

\section{Additional information}

Extended data is available for this paper at https://doi.org/10.1038/s44161-021-00016-2. Supplementary information The online version contains supplementary material available at https://doi.org/10.1038/s44161-021-00016-2.

Correspondence and requests for materials should be addressed to Masayuki Yazawa.

Peer review information Nature Cardiovascular Research thanks the anonymous reviewers for their contribution to the peer review of this work.

Reprints and permissions information is available at www.nature.com/reprints.

Publisher's note Springer Nature remains neutral with regard to jurisdictional claims in published maps and institutional affiliations.

(c) The Author(s), under exclusive licence to Springer Nature Limited 2022 
a

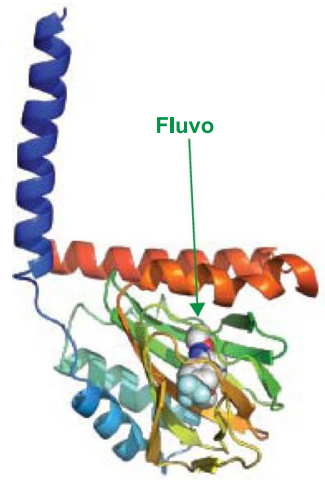

b

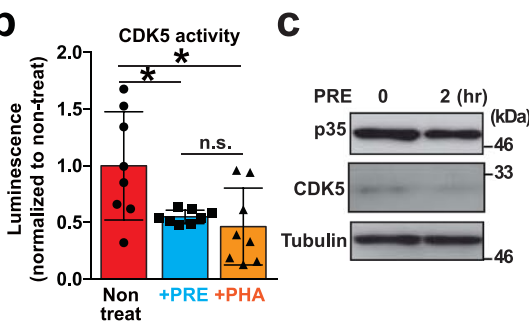

h

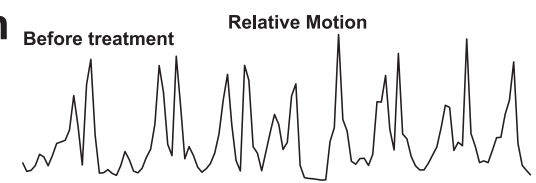

After $5 \mu$ M PRE-084 treatment ( $2 \mathrm{hr}$ )

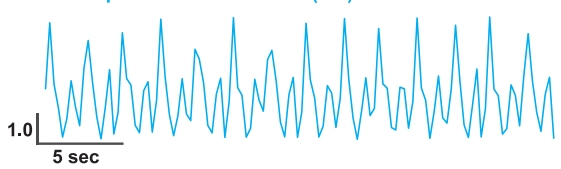

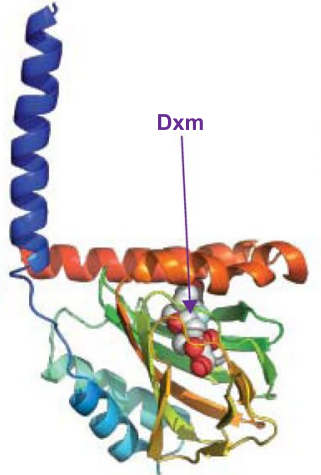

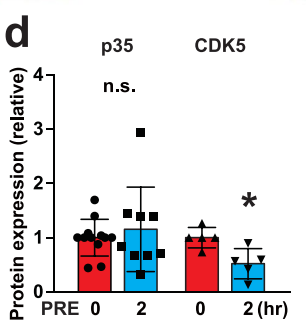

i

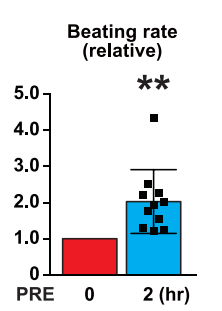

k

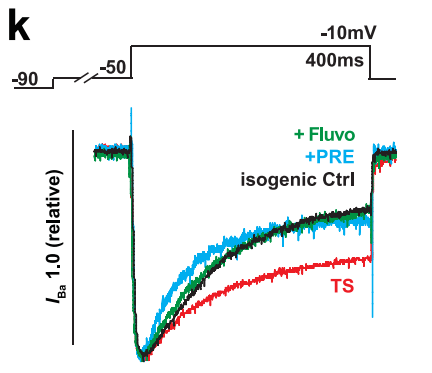

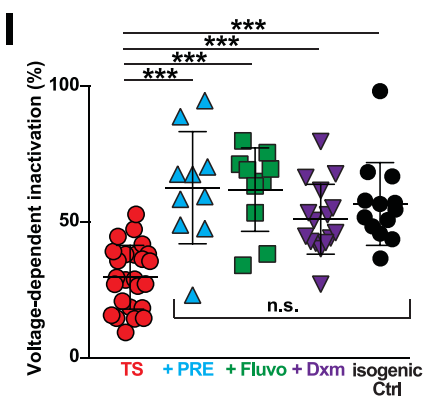

f

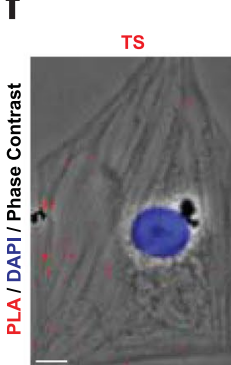

PLA: SIGMAR1-CDK5
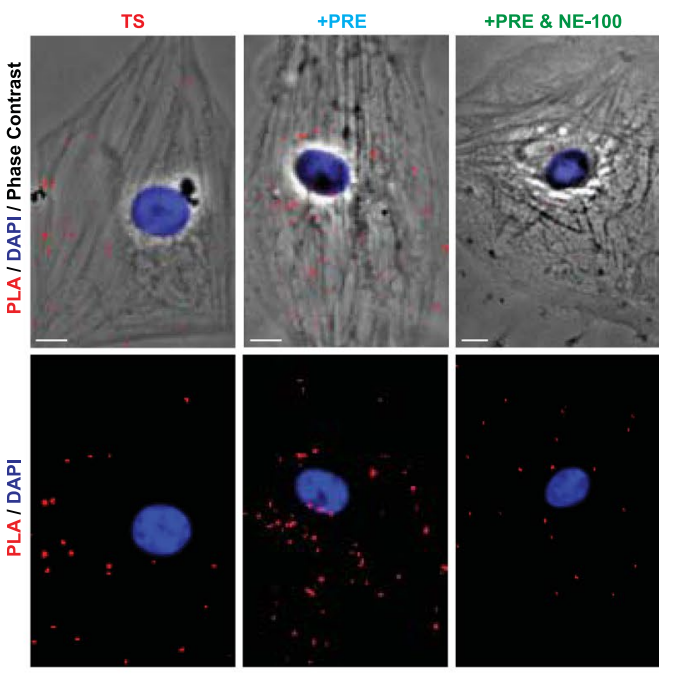

g

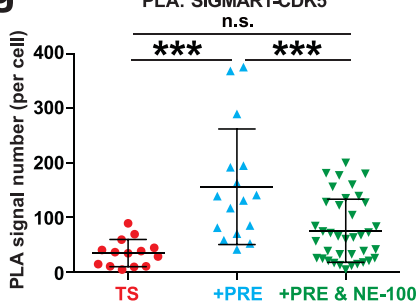

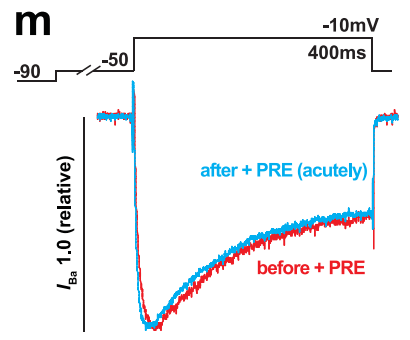

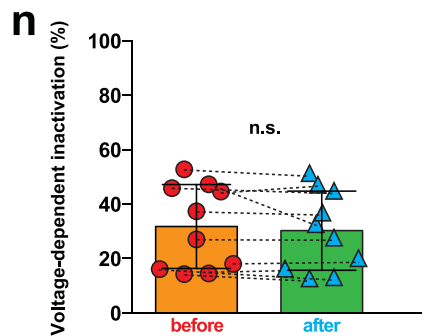

Extended Data Fig. 1 | The effect of SIGMAR1 agonists on CDK5, contraction and calcium channel in Timothy syndrome iPSC-derived cardiomyocytes.

(a) Docking models of SIGMAR1-Fluvo, -Dxm and -PRE. (b) PRE-084 ( $5 \mu \mathrm{M}, 2 \mathrm{hr})$ reduced CDK5 kinase activity in Timothy syndrome (TS) cardiomyocytes. PHA-793887 (PHA, $5 \mu \mathrm{M})$, a CDK5 inhibitor, was used as positive control for the assay $(n=8 /$ group). (c-e) The effects of PRE ( $5 \mu \mathrm{M}$, $2 \mathrm{hr}$ ) on CDK5R1/p35 protein (c, d, $n=12$ for baseline and $n=9$ for $2 \mathrm{hr}$ after treatment) and CDK5 protein (c, $\mathbf{d}, n=5 /$ group) and mRNA (e, $n=4 / g r o u p)$. (f) Representative epi-fluorescent and phase-contrast images of TS cardiomyocytes without treatment, with PRE-084 (+PRE, $5 \mu \mathrm{M}, 2 \mathrm{hr})$ or PRE-084 and NE-100 treatment (+PRE \& NE-100, both $5 \mu \mathrm{M}, 2 \mathrm{hr}$ ) from proximity ligation assay (PLA, SIGMAR1-CDK5, red, DAPI, blue). Scale bar, 10 $\mu \mathrm{m}$. (g) SIGMAR1-CDK5 PLA quantification in PRE-treated $(n=16)$, PRE\&NE-100-treated $(n=38)$ and non-treated TS cardiomyocytes $(n=14)$. (h) Representative traces of relative motion analysis of TS cardiomyocyte contractions before (black) and after 2-hr PRE-084 treatment (blue). The representative traces were obtained from Supplementary Movies 1 and 2. (i-j) Relative changes of beating rate (i) and irregularity (j) $(n=11)$ of TS cardiomyocytes after PRE-084 treatment. (k) Representative traces of $\mathrm{Ba}^{2+}$ currents in TS cardiomyocytes without treatment or treated with PRE-084 (+PRE), or fluvoxamine (+Fluvo) (each, $5 \mu \mathrm{M}, 2 \mathrm{hr}$ ) and in isogenic control cardiomyocytes (Ctrl). +Dxm representative trace is shown in Fig. 2f. (I) Voltage-dependent calcium channel inactivation was significantly enhanced by PRE $(n=10)$, Fluvo $(n=10)$ and Dxm $(n=16)$ treatment in TS cardiomyocytes compared to non-treated cells $(n=25)$. n.s., no significant differences between + PRE, +Fluvo, + Dxm and isogenic Ctrl groups $(n=13)$. ( $\mathbf{m})$ Representative traces of Ba ${ }^{2+}$ currents in TS cardiomyocytes before treatment and acutely treated with PRE ( $5 \mu \mathrm{M}, \sim 5-10 \mathrm{mins})$. (n) Voltage-dependent calcium channel inactivation was not significantly changed by acute PRE treatment in TS cardiomyocytes $(n=10)$. All data are mean \pm s.d. One-way ANOVA with Tukey's multiple comparisons was used for b, g and One-way ANOVA with Sidak's multiple comparisons was used for I. Paired two-tailed Student's $t$-test was used for $\mathrm{i}, \mathrm{j}$, $\mathrm{n}$, and unpaired two-tailed Student's $t$-test was used for d, e. ${ }^{\star} P<0.05,{ }^{\star \star} P<0.01,{ }^{\star \star \star} P<0.001$, n.s., not significant. Cell samples from at least two independent differentiations were used. 

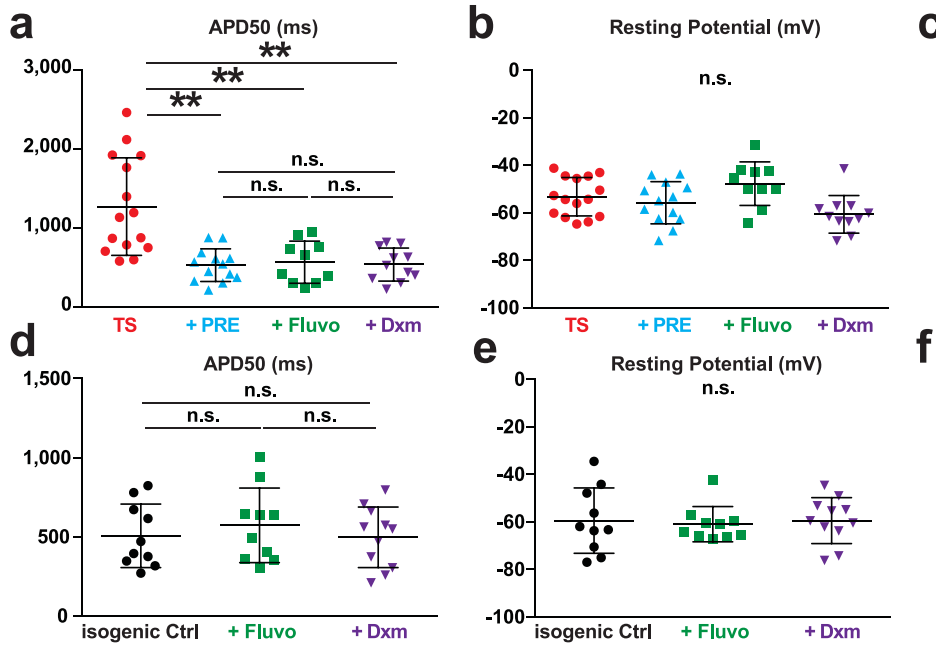

C Peak Amplitude (mV)
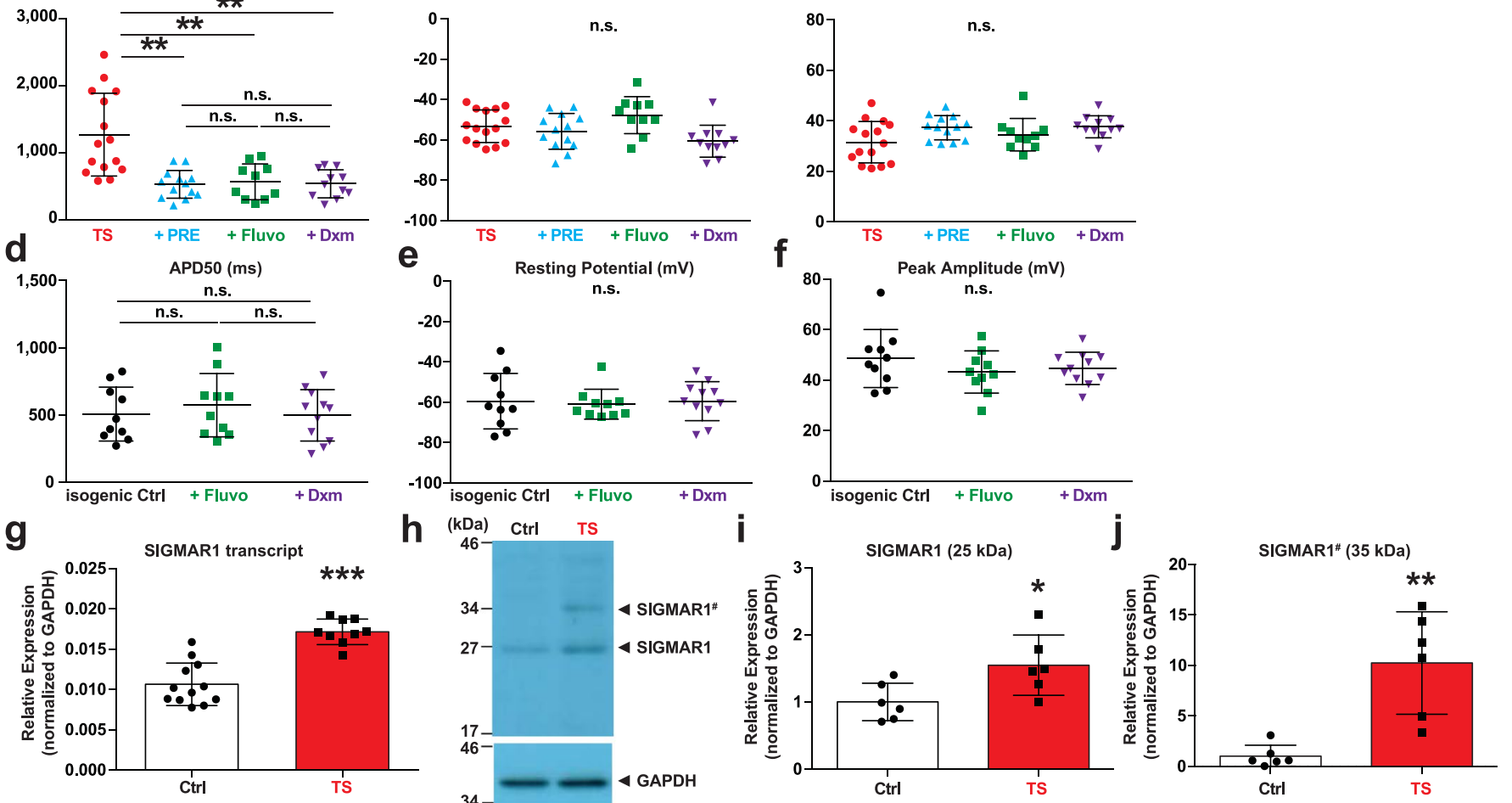

k
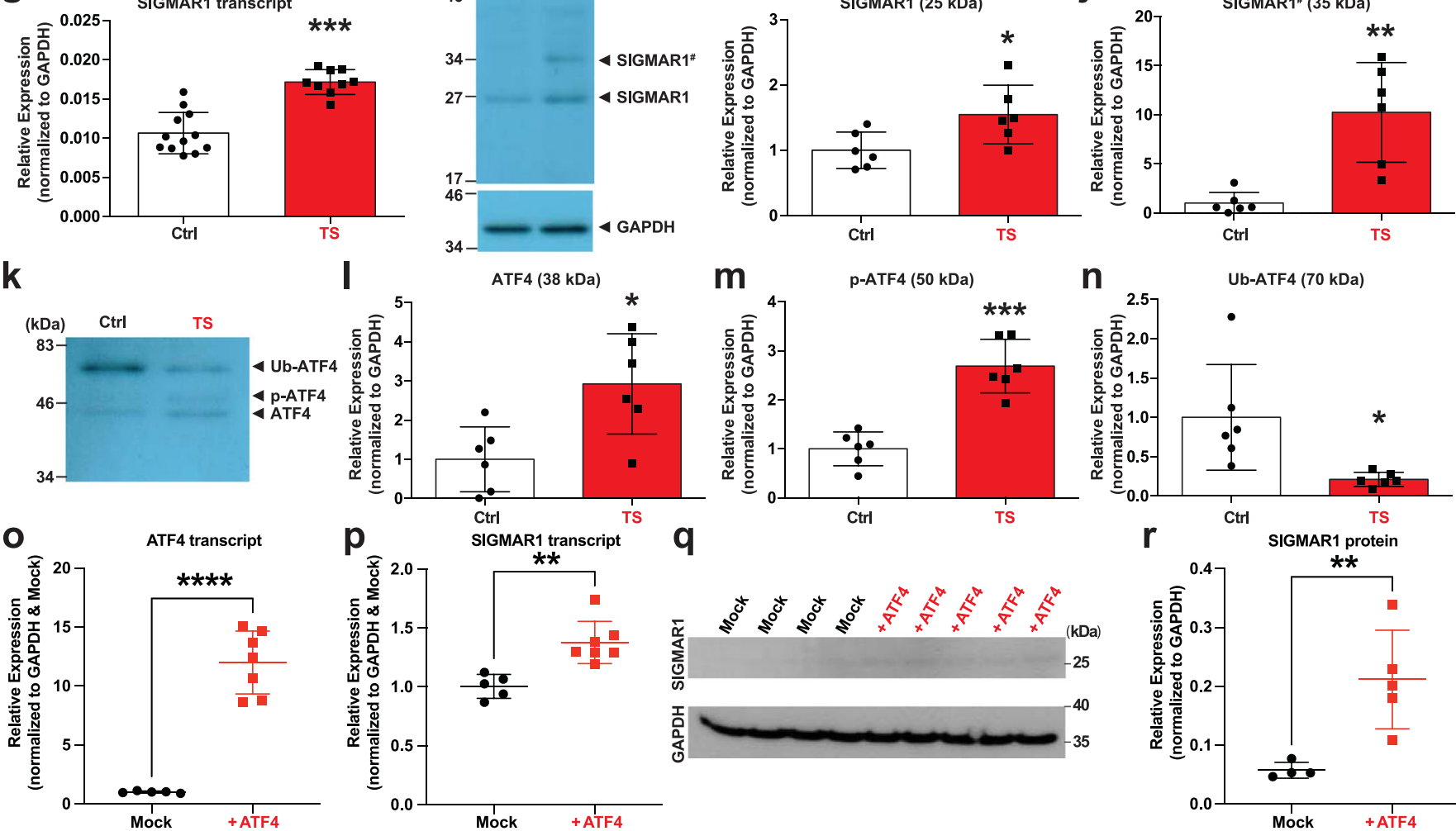

Extended Data Fig. 2 | The effect of SIGMAR1 agonists on action potential and SIGMAR1 and ATF4 expression profiling in Timothy syndrome

iPSC-derived cardiomyocytes. (a-c) Action potential parameters, APD50 (a), resting potential (b) and peak amplitude (c) in Timothy syndrome (TS) cardiomyocytes without treatment $(n=15)$ or treated for 2 hrs with $5 \mu$ M SIGMAR1 agonists, PRE-084 (+PRE, $n=13)$, fluvoxamine (+Fluvo, $n=10)$ or dextromethorphan (+Dxm, $n=11)$. APD90 is shown in Fig. 1d. (d-f) Action potential parameters, APD50 (d), resting potential (e) and peak amplitude (f) in isogenic control (Ctrl) cardiomyocytes without treatment $(n=10)$ and treated for 2 hrs with $5 \mu$ M SIGMAR1 agonists, Fluvo $(n=10)$ or Dxm $(n=11)$. (g) Quantification of human SIGMAR1 transcripts (normalized to GAPDH) in Timothy syndrome ( $n=9$ from two independent lines) and control cardiomyocytes ( $n=12$ from four independent lines). (h) Representative immunoblots of human SIGMAR1 and GAPDH protein using lysates from TS and control iPSC-derived cardiomyocytes. (i-j) Quantification of SIGMAR1 $25 \mathrm{kDa}$ protein band (i) and $35 \mathrm{kDa}$ protein expression (j, normalized to GAPDH) in TS iPSC-derived cardiomyocytes compared to the isogenic Ctrl ( $n=6 /$ group). The molecular weight of SIGMAR1 is $\sim 25 \mathrm{kDa}$ while the $35 \mathrm{kDa}$ band (\#) has been reported previously and might be a dimer of a full-length SIGMAR1 with a SIGMAR1 splice variant ${ }^{59}$. (k) Representative immunoblots of human ATF4 using the same lysates from TS and isogenic Ctrl iPSC-derived cardiomyocytes shown in Extended Data Fig. 2h. (I-n) Quantification of ATF4 38 kDa protein band (I, non-modified), $50 \mathrm{kDa}$ ( $\mathbf{m}$, phosphorylated) and $70 \mathrm{kDa}$ protein expression (n, ubiquitinated, normalized to GAPDH) in TS iPSC-derived cardiomyocytes compared to the isogenic $\mathrm{Ctrl}(n=6 /$ group). (o-r) ATF4 overexpression (o) significantly increased SIGMAR1 transcription (p) and protein expression ( $\mathbf{q}, \mathbf{r})$ in normal human cardiomyocytes transfected with ATF4 plasmid (+ ATF4, o\&p, $n=7, r, n=5$ ). The cardiomyocytes were harvested 24 hr after the lipofection. The empty vector was used as a negative control (Mock, o\&p, $n=5, r, n=4$ ). (q) Representative immunoblots of human SIGMAR1 and GAPDH using the lysate from the transfected cardiomyocytes. All data are mean \pm s.d. One-way ANOVA with Tukey's multiple comparisons was used for a-f and unpaired two-tailed Student's $t$-test was used for $\mathrm{g}, \mathrm{i}, \mathrm{j}, \mathrm{l}, \mathrm{m}, \mathrm{n}, \mathrm{o}, \mathrm{p}, \mathrm{r} .{ }^{\star} P<0.05,{ }^{\star \star} P<0.01,{ }^{\star \star \star} P<0.001$, ${ }^{\star \star \star \star} P<0.0001$, n.s., not significant. Cell samples from at least two independent differentiations were used. 

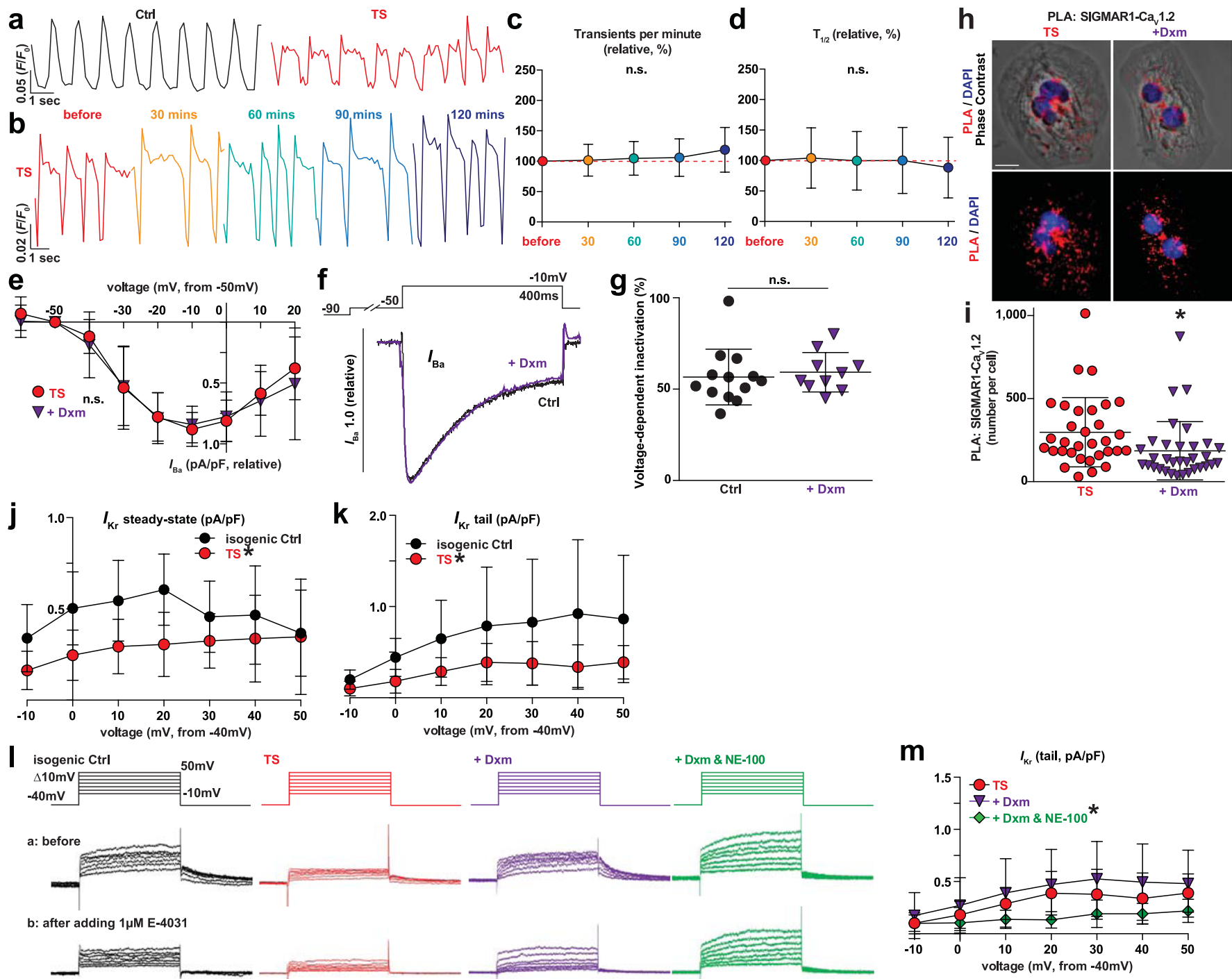

b: after adding $1 \mu \mathrm{M} \mathrm{E}-4031$
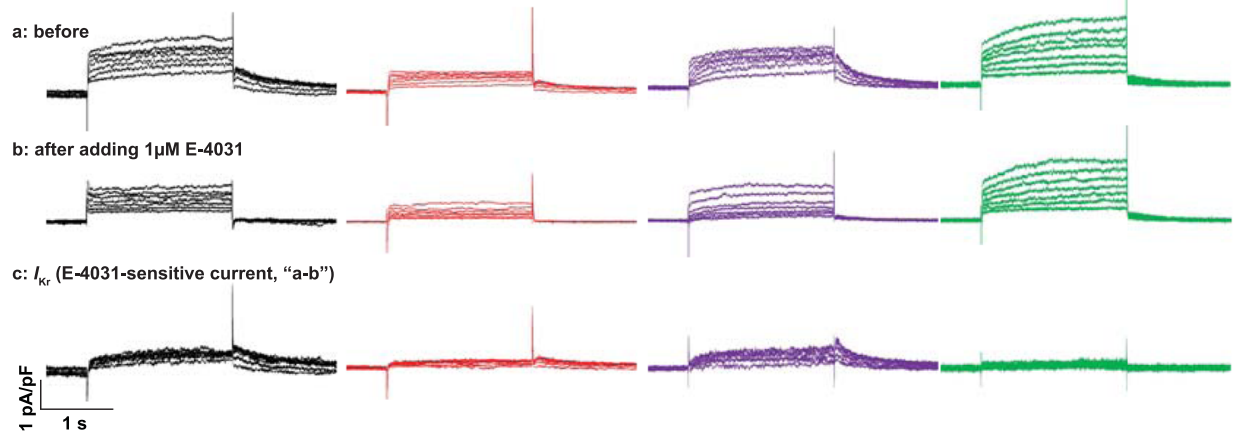

Extended Data Fig. 3 | The effect of dextromethorphan on cardiac calcium and hERG channels in Timothy syndrome iPSC-derived cardiomyocytes. (a-b) Representative traces of time-course calcium imaging in spontaneously contracting isogenic control (Ctrl, black) and Timothy syndrome (TS, red) cardiomyocytes. (c-d) Calcium transient frequency (c) and duration (d) analysis in TS cardiomyocytes during the 2-hr imaging ( $n=32$ ). (e) Currentvoltage relationship of $\mathrm{Ba}^{2+}$ recordings in Dxm-treated $(n=12)$ and non-treated TS cardiomyocytes $(n=13)$. ( $\left.\mathbf{f}\right)$ Representative traces of $\mathrm{Ba}^{2+}$ currents in isogenic control cardiomyocytes without treatment (Ctrl) or treated with dextromethorphan (+Dxm). ( $(\mathbf{g})$ Voltage-dependent calcium channel inactivation was not altered by $\operatorname{Dxm}(n=10)$ in the isogenic control cardiomyocytes compared to non-treated cells $(n=13)$. (h) Representative epi-fluorescent and phase-contrast images of TS cardiomyocytes without and with Dxm (+Dxm) from proximity ligation assay (PLA, SIGMAR1-Cav1.2, red, DAPI, blue). Scale bar, $10 \mu \mathrm{m}$. (i) SIGMAR1-Ca 1.2 PLA quantification in Dxm-treated $(n=33)$ and non-treated TS cardiomyocytes $(\mathrm{TS}, n=32)$. (j) $I_{\mathrm{kr}}$ current steady-state amplitudes were significantly reduced in TS cardiomyocytes $(n=10)$ compared to isogenic control $(n=10)$ at $-10,0$ and $10 \mathrm{mV}$ steps from $-40 \mathrm{mV}$ hold. (k) $I_{\mathrm{Kr}}$ tail currents were significantly reduced in TS cardiomyocytes $(n=10)$ compared to isogenic control $(n=10)$ at $-10,0,10$ and $20 \mathrm{mV}$ steps.

(I) Representative traces of $I_{\mathrm{Kr}}$ currents (E-4031-sensitive) in isogenic Ctrl cardiomyocytes (black) and TS cardiomyocytes treated with Dxm (purple), Dxm \& NE-100 (green) or without treatment (red, TS). The $I_{\mathrm{Kr}}$ traces are shown in Fig. 2h. ( $\mathbf{m}$ ) There was no significant difference in $I_{\mathrm{Kr}}$ current (tail) between Dxm-treated $(n=9)$ and non-treated TS cardiomyocytes $(n=10)$ while Dxm \& NE-100 $(n=10)$ significantly reduced tail currents compared to Dxm at 10 , 20, 30, 40 and $50 \mathrm{mV}$ steps and also to non-treated TS at $20 \mathrm{mV}$ step from $-40 \mathrm{mV}$ hold. All data are mean \pm s.d. The treatment of Dxm or NE-100 for all experiments was $5 \mu \mathrm{M}, 2 \mathrm{hrs}$. One-way ANOVA with Tukey's multiple comparisons was used for $\mathrm{c}$, $\mathrm{d}$ between the time points to before and used for $\mathrm{m}$ at each voltage step. Unpaired two-tailed Student's $t$-test were used for $\mathrm{g}$, i between the groups and used for e, j, $\mathrm{k}$ at each voltage step. ${ }^{\star} P<0.05$, n.s., no significant. The samples were from at least two independent differentiations. 

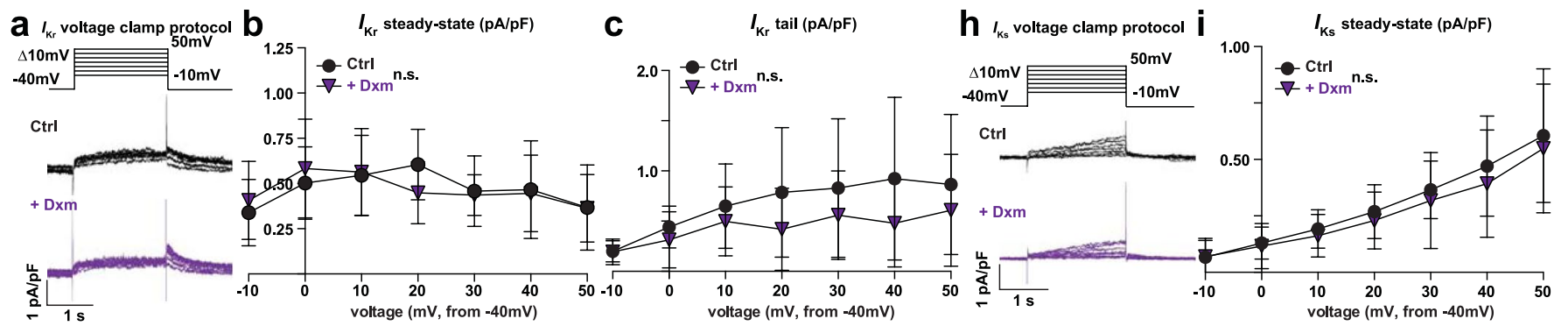

d

PLA: SIGMAR1-hERG

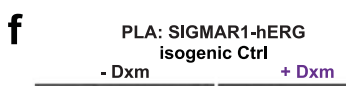

j

PLA: SIGMAR1-Kv7.1

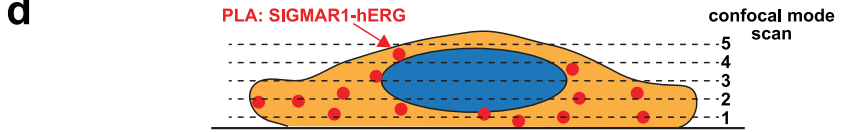

e 5
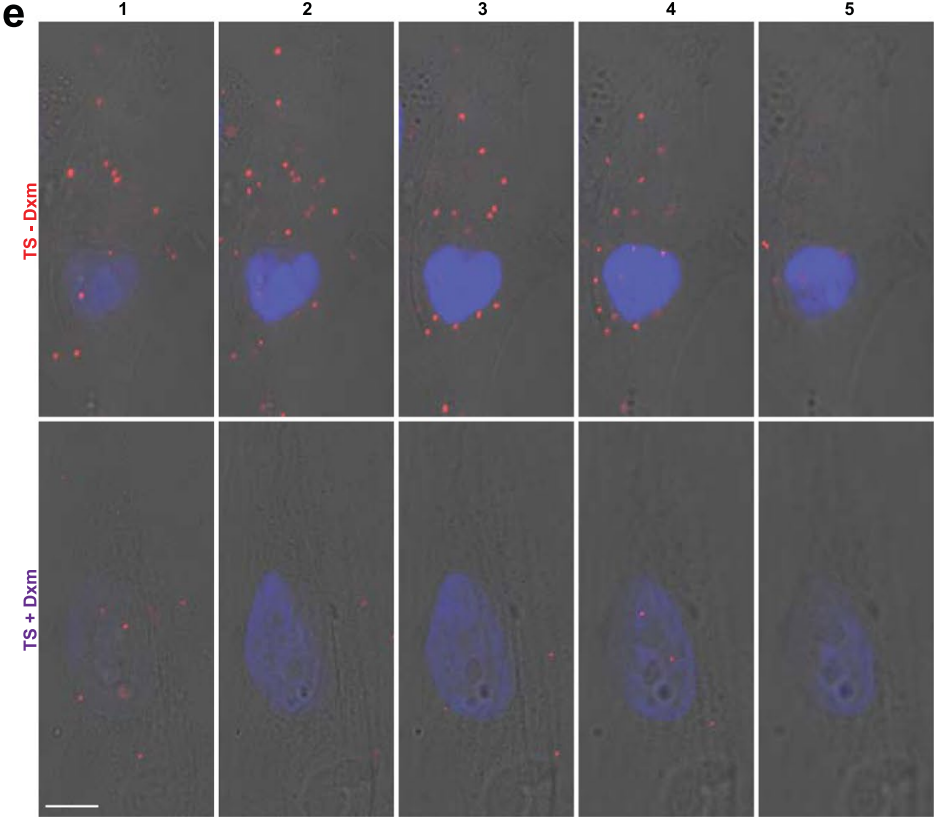

PLA: SIGMAR1-hERG / DAPI / Bright Field

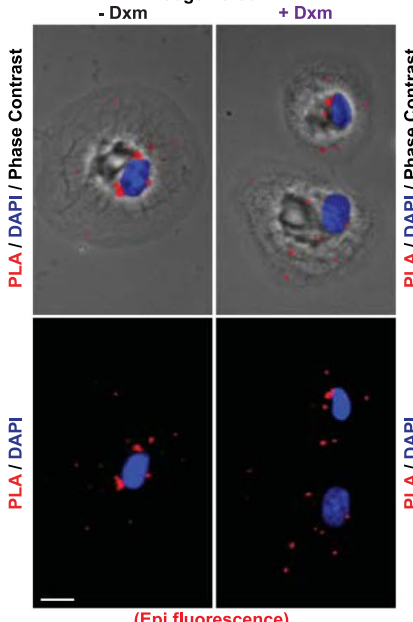

(Epi fluorescence)

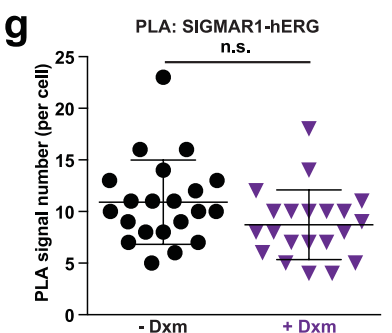

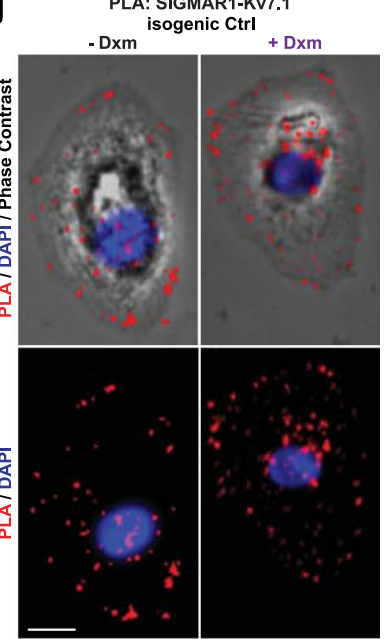

k

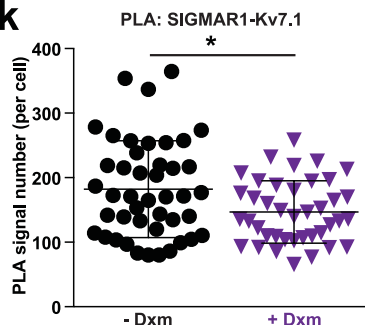

Extended Data Fig. 4 | The effect of dextromethorphan on potassium channels in human cardiomyocytes derived from isogenic control iPSCs.

(a) Representative traces of $I_{\mathrm{Kr}}$ current (E-4031-sensitive) in isogenic control (Ctrl) cardiomyocytes treated with Dxm ( $5 \mu \mathrm{M}, 2 \mathrm{hr}$ ) or without treatment. (b-c) $I_{\mathrm{Kr}}$ current amplitude (b) and tail $I_{\mathrm{Kr}}$ current (c) were not significantly changed by Dxm in the isogenic Ctrl cardiomyocytes ( $n=10 / g r o u p$ ).

(d) Schematic representation of confocal imaging for proximity ligation assay (PLA, SIGMAR1-hERG, red) in human iPSC-derived cardiomyocytes. (e) Representative confocal fluorescent images of TS cardiomyocytes used for PLA (SIGMAR1-hERG, red) and DAPI (blue) staining. Dxm treatment (+Dxm, $5 \mu \mathrm{M}, 2 \mathrm{hr}$ ) was conducted to examine the effect of Dxm on PLA signals in TS cardiomyocytes. \#3 images are used in Fig. 2j. Scale bar, 10 $\mu \mathrm{m}$. (f) Representative epi-fluorescent and phase-contrast images of isogenic Ctrl cardiomyocytes used for PLA (red, SIGMAR1-hERG) and DAPI (blue) staining. Dxm treatment ( + Dxm, $5 \mu \mathrm{M}, 2 \mathrm{hr}$ ) was conducted to examine the effect of Dxm on PLA signals in the cardiomyocytes. Scale bar, $10 \mu \mathrm{m}$. (g) Quantification of PLA signal number of SIGMAR1-hERG in Dxm-treated $(n=21)$ and non-treated $(n=21)$ isogenic Ctrl cardiomyocytes. (h) Representative traces of $I_{\mathrm{Ks}}$ current (Chromanol 293B-sensitive) in isogenic control (Ctrl) cardiomyocytes treated with Dxm or without treatment. (i) $I_{\mathrm{Ks}}$ current amplitude analysis in the isogenic Ctrl cardiomyocytes with $\operatorname{Dxm}(5 \mu \mathrm{M}, 2 \mathrm{hr}, n=9)$ or without treatment $(n=10)$. (j) Representative epi-fluorescent and phase-contrast images of isogenic Ctrl cardiomyocytes used for PLA (red, SIGMAR1-K 7.1 ) and DAPI (blue) staining. Dxm treatment (+Dxm, $5 \mu \mathrm{M}, 2 \mathrm{hr}$ ) was conducted to examine the effect of Dxm on PLA signals in the cardiomyocytes. Scale bar, 10 $\mu \mathrm{m}$. (k) Quantification of PLA signal number of SIGMAR1-K 7.1 in Dxm-treated $(n=40)$ and non-treated $(n=44)$ isogenic Ctrl cardiomyocytes. All data are mean \pm s.d. Unpaired two-tailed Student $t$-test was used for g,k between the groups, and for b,c,i at each voltage step. ${ }^{\star} P<0.05$. n.s., not significant. The cell samples were from at least two independent differentiations. 

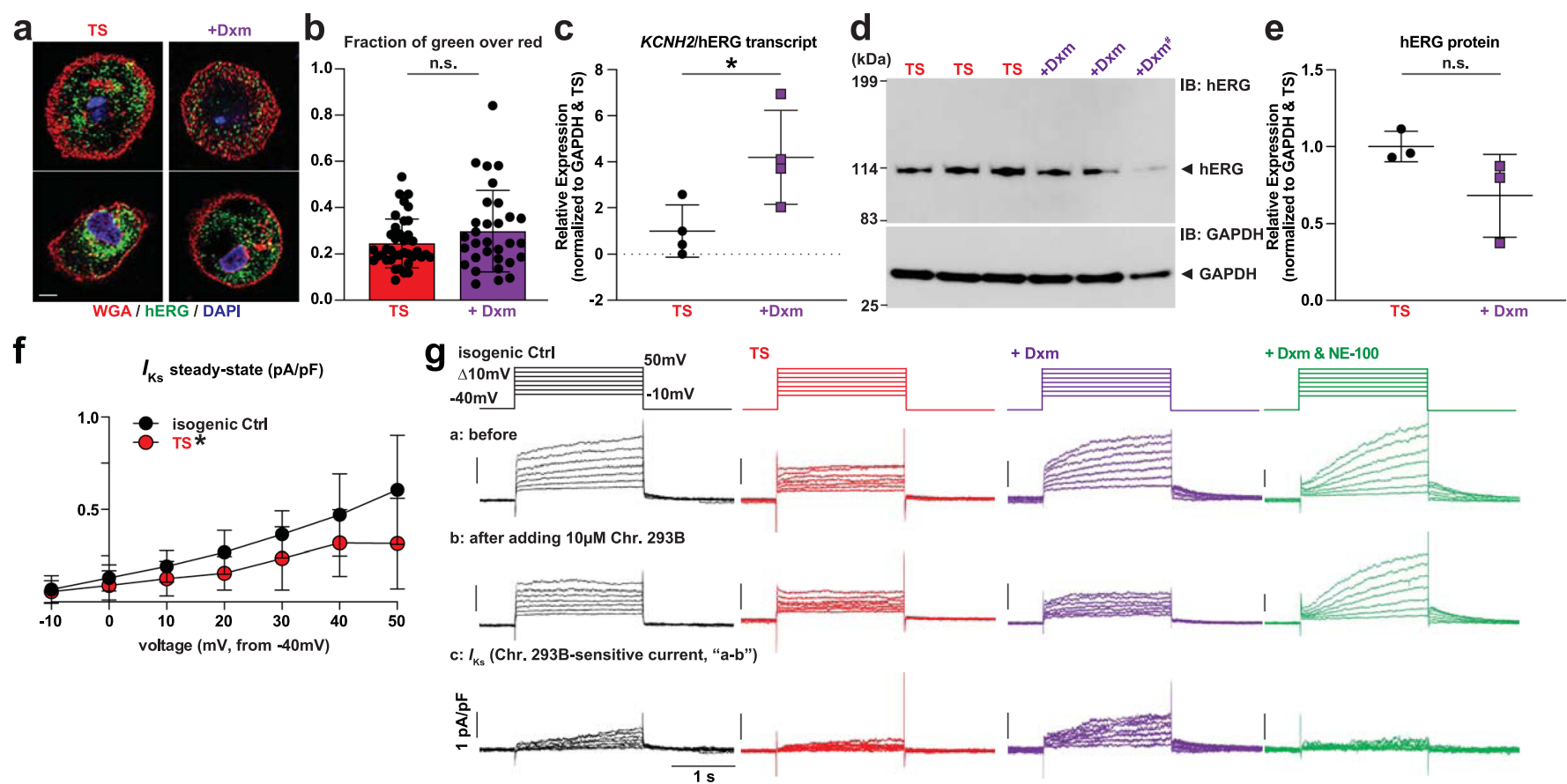

h
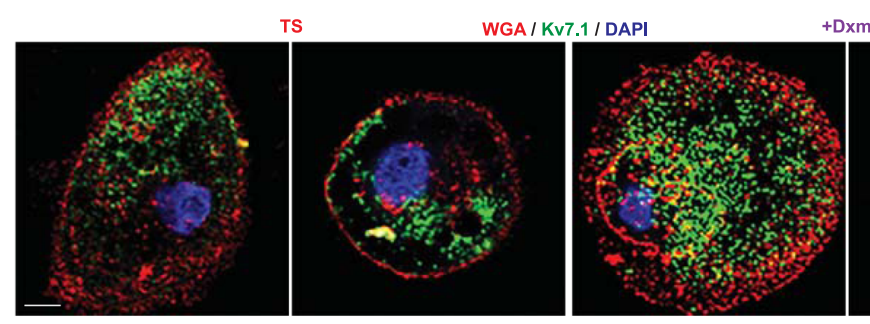
Dxm
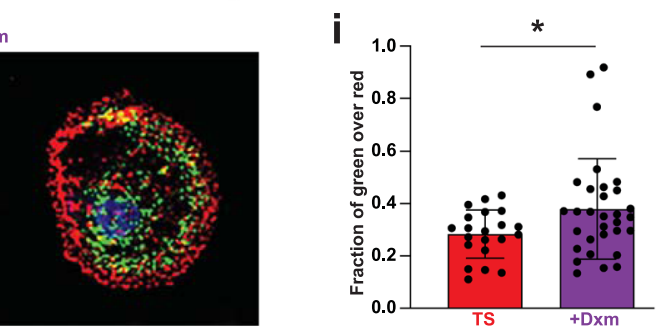

j
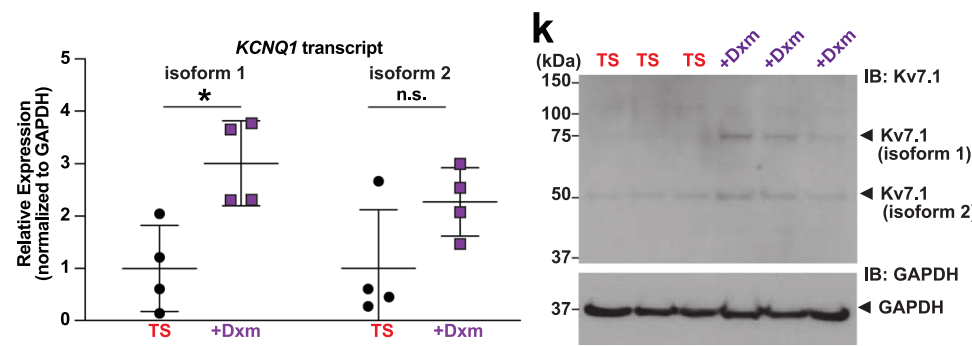

I

m

Extended Data Fig. 5 | The effect of dextromethorphan on potassium channels in Timothy syndrome iPSC-derived cardiomyocytes. (a) Representative confocal fluorescent images of Timothy syndrome cardiomyocytes without treatment (left, TS) and with dextromethorphan (+Dxm). Scale bar, 5 $\mu$ m. (b) Quantification of fraction of green (hERG) over red (WGA) fluorescence signals in TS cardiomyocytes without treatment $(n=38)$ and with Dxm $(n=31)$. (c) Quantification of $K C N H 2$ transcript in TS cardiomyocytes with and without Dxm ( $n=4 /$ group). (d) Representative immunoblots of human hERG and GAPDH protein using lysates from non-treated and Dxm-treated TS cardiomyocytes. (e) Quantification of hERG protein expressions (normalized to GAPDH) in non-treated and Dxm-treated TS cardiomyocytes ( $n=3 /$ group). The trend towards a decreased hERG protein expression in the treated group might be driven by one sample (\#) with a reduced loading amount compared with others. (f) $I_{\mathrm{Ks}}$ current steady-state amplitudes were significantly reduced in TS cardiomyocytes $(n=10)$ compared to isogenic control $(n=10)$ at 20 and $50 \mathrm{mV}$ steps from $-40 \mathrm{mV}$ hold. (g) Representative traces of $I_{\mathrm{Ks}}$ currents (Chromanol 293B-sensitive) in isogenic Ctrl cardiomyocytes (black) and TS cardiomyocytes treated with Dxm (purple), Dxm and $\mathrm{NE}-100$ (green) or without treatment (red, TS). The $I_{\mathrm{Ks}}$ traces are shown in Fig. 2l. (h) Representative confocal fluorescent images of Timothy syndrome cardiomyocyte without treatment (left, TS) and treated with dextromethorphan (+Dxm). Scale bar, 5 $\mathrm{mm}$. (i) Quantification of fraction of green $\left(\mathrm{K}_{\mathrm{v}} 7.1\right)$ over red (WGA) fluorescence signals in TS cardiomyocytes without treatment $(n=21)$ and treated with Dxm $(n=31)$. (j) Quantification of KCNQ1 transcripts (isoform 1 and 2) in TS cardiomyocytes without treatment and treated with Dxm ( $n=4 /$ group). (k) Representative immunoblots of human $K_{\mathrm{v}} 7.1$ and GAPDH protein using lysates from non-treated and Dxm-treated TS cardiomyocytes. (I-m) Quantification of $\mathrm{K}_{\mathrm{v}} 7.1$ isoform 1 (I) and isoform 2 protein expressions ( $\mathbf{m}$, normalized to GAPDH) in non-treated and Dxm-treated TS cardiomyocytes ( $n=3 /$ group). All data are mean \pm s.d. The treatment of Dxm or NE-100 for all experiments was $5 \mu \mathrm{M}, 2 \mathrm{hrs}$. Unpaired two-tailed Student's $t$-test was used for b, c, e, i, j, l, $m$ between the groups and used for $\mathrm{f}$ at each voltage step. ${ }^{\star} P<0.05$. n.s., not significant. The cell samples were from at least two independent differentiations. 
a LQTS1 (KCNQ1 G269S) LQTS1 isogenic CtrI

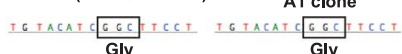
Gly
Ser
$(A \subseteq C)$

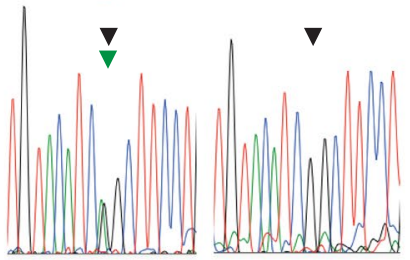

C
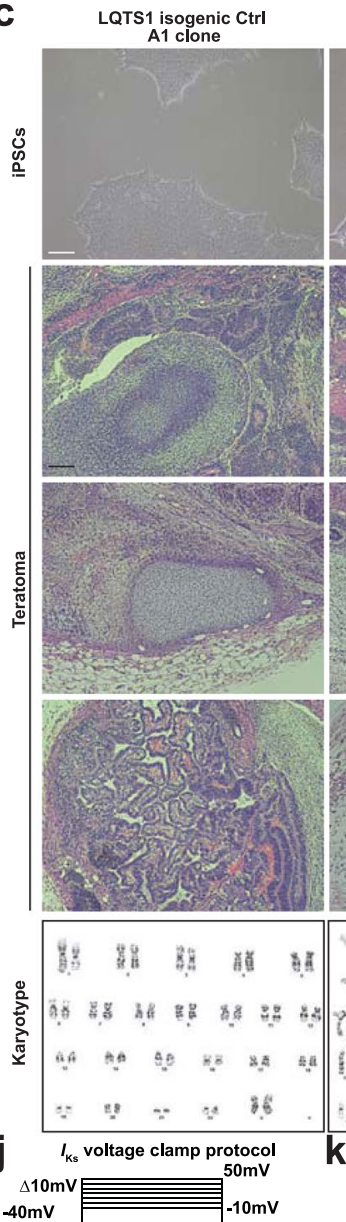

isogenic Ctrl
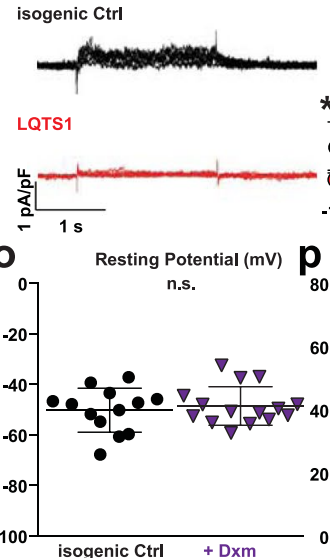
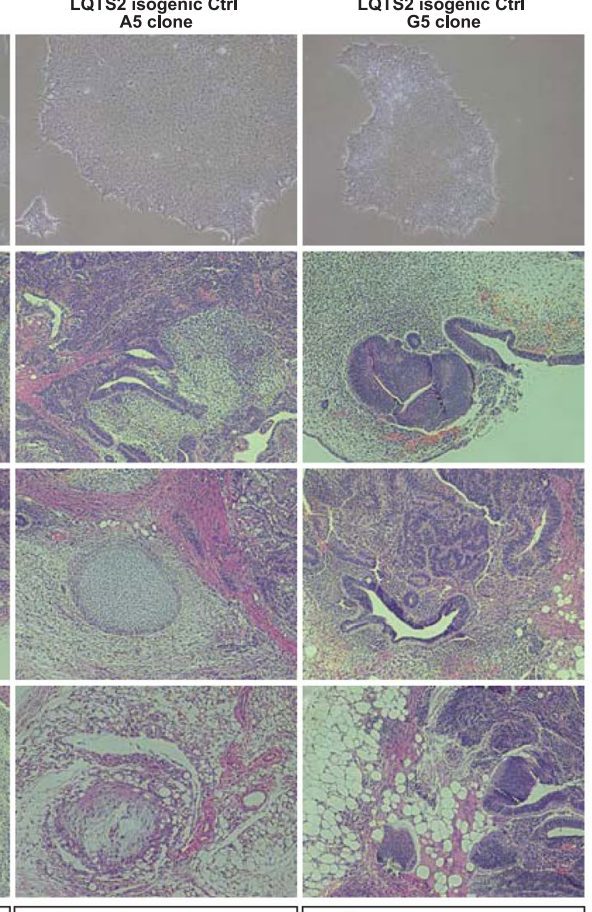

is $x$
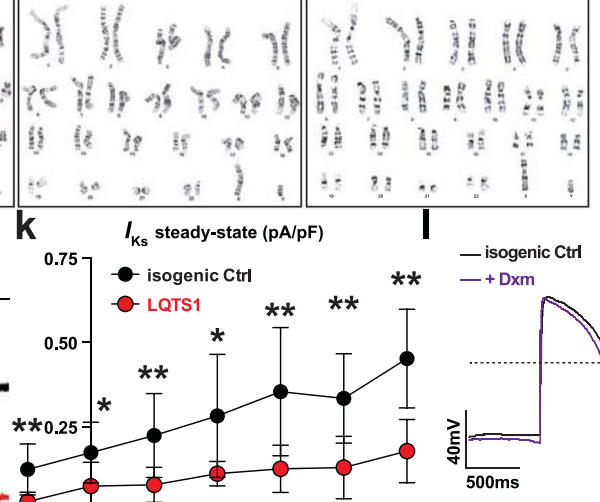

LQTS2 isogenic Ctrl

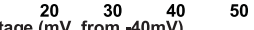

Peak Amplitude $(\mathrm{mV}) \quad \mathbf{Q} I_{\mathrm{kr}}$ voltage clamp protocol
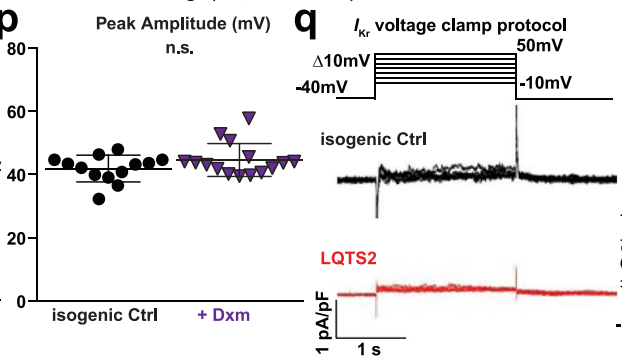
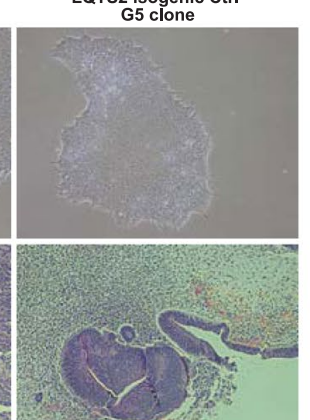

e - isogenic Ctrl
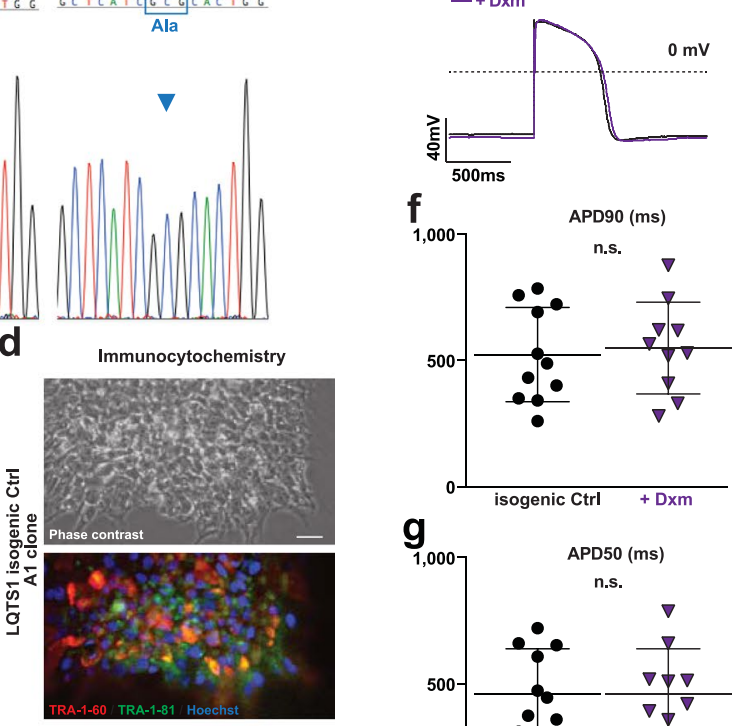

d Immunocytochemistry
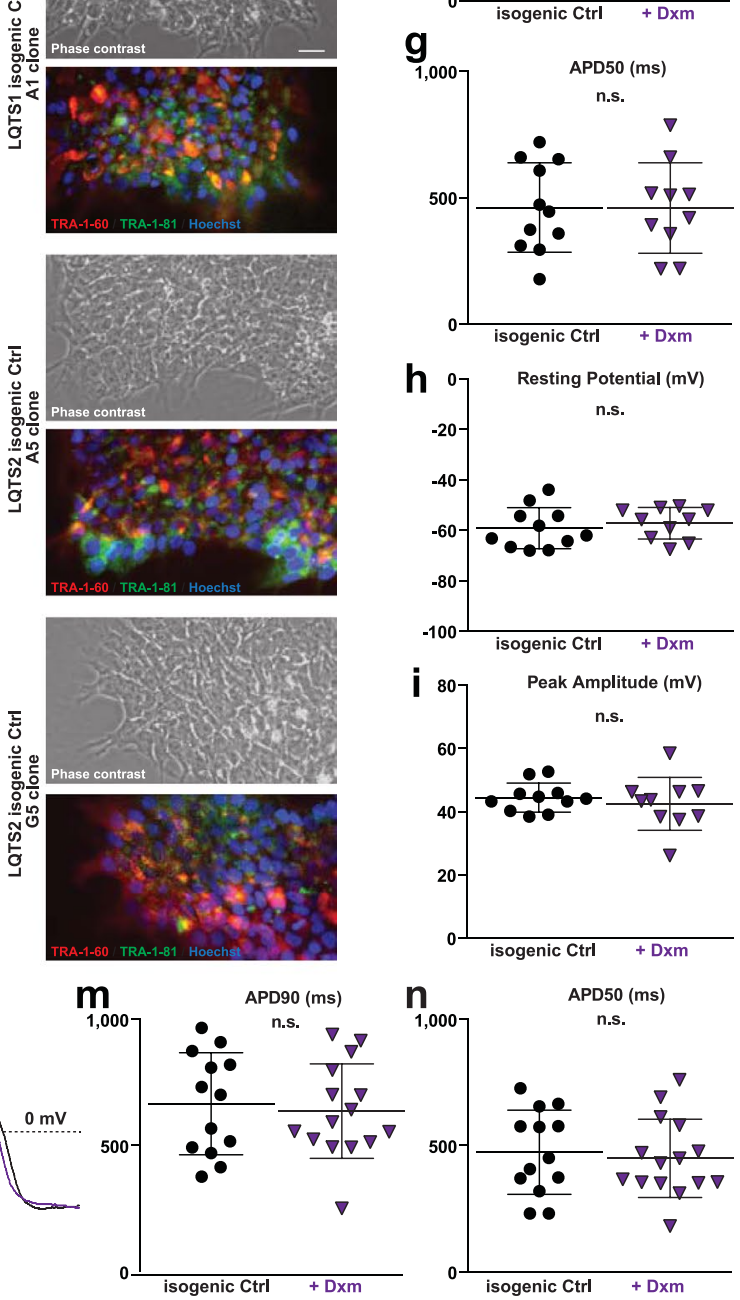

$\mathbf{S}$
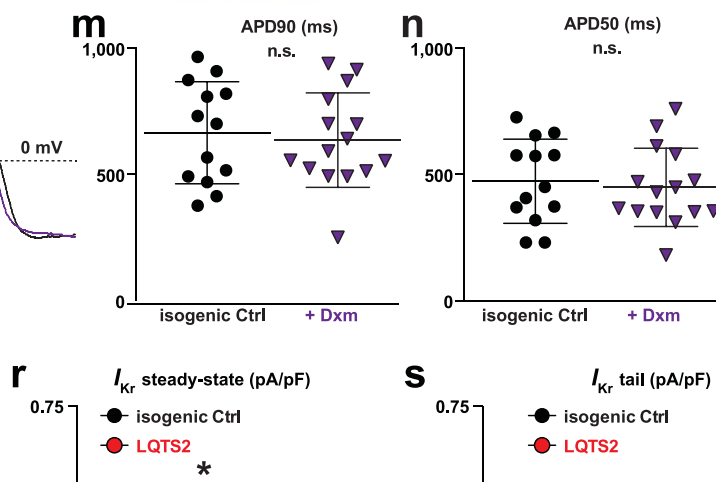

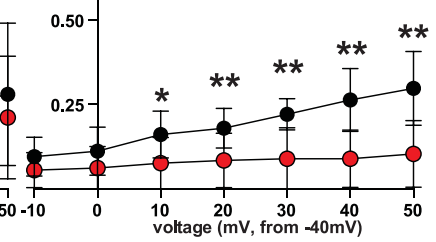

Extended Data Fig. 6 | See next page for caption. 
Extended Data Fig. 6 | Generation of isogenic controls from long QT syndrome type 1 and 2 iPSCs. (a-b) Sequencing results confirmed the successful generation of isogenic control (Ctrl) iPSC lines from long QT syndrome type 1 (a) and type 2 (b). (c) Characterization of the isogenic Ctrl iPSC lines (top, phase contrast image; middle, teratoma formation assay). Scale bar, 200 $\mu \mathrm{m}$. The isogenic Ctrl iPSC demonstrated normal karyotype (bottom). (d) Representative images of TRA-1-60 (red)/TRA-1-81 (green)/Hoechst (nuclei, blue) stain in isogenic Ctrl iPSC lines. Scale bar, 20 $\mu$ m. (e) Representative traces of $0.5 \mathrm{~Hz}$-paced action potentials in LQTS1 patient-specific isogenic Ctrl iPSC-derived cardiomyocytes without treatment or treated with Dxm. (f-i) Analysis of action potential parameters, APD90 (f), APD50 (g), resting potential (h) and peak amplitude (i), in isogenic Ctrl cardiomyocytes without treatment $(n=11)$ or treated with Dxm $(n=10)$. (j) Representative traces of $I_{\mathrm{Ks}}$ currents (Chromanol-293B-sensitive) in long QT syndrome type 1 (LQTS1) patient-specific cardiomyocytes and the isogenic Ctrl cardiomyocytes. (k) $I_{\mathrm{Ks}}$ current steady-state amplitudes were significantly reduced in LQTS1 cardiomyocytes $(n=10)$ compared to the isogenic control $(n=11)$ at all voltage steps from $-40 \mathrm{mV}$ hold. (I) Representative traces of $0.5 \mathrm{~Hz}$-paced action potentials in long QT syndrome type 2 (LQTS2) patient-specific isogenic Ctrl iPSC-derived cardiomyocytes without treatment or treated with Dxm. $(\mathbf{m}-\mathbf{p})$ analysis of action potential parameters, APD90 (m), APD50 (n), resting potential (o) and peak amplitude (p), in the isogenic Ctrl cardiomyocytes without treatment $(n=13)$ or treated with Dxm $(n=15)$. (q) Representative traces of $I_{\mathrm{kr}}$ currents (E4031-sensitive) in LQTS2 patient-specific cardiomyocytes and the isogenic Ctrl cardiomyocytes. $(\mathbf{r}) I_{\mathrm{kr}}$ current steady-state amplitudes were significantly reduced in LQTS2 cardiomyocytes $(n=10)$ compared to the isogenic control $(n=12)$ at 10 and $20 \mathrm{mV}$ steps from $-40 \mathrm{mV}$ hold. (s) $I_{\mathrm{kr}}$ tail currents were significantly reduced in LQTS2 cardiomyocytes compared to the isogenic control at 10, 20,30, 40 and $50 \mathrm{mV}$ steps from $-40 \mathrm{mV}$ hold. All data are mean \pm s.d. The treatment of Dxm for the experiments was $5 \mu \mathrm{M}, 2 \mathrm{hrs}$. Unpaired two-tailed Student's t-test were used for $\mathrm{f}, \mathrm{g}, \mathrm{h}, \mathrm{i}, \mathrm{m}, \mathrm{n}, \mathrm{o}, \mathrm{p}$ between the groups and for $\mathrm{k}, \mathrm{r}$, $\mathrm{s}$ at each voltage step. ${ }^{\star} P<0.05$, $\star \star P<0.01$, n.s., not significant. The cardiomyocytes were from at least two independent differentiations. 

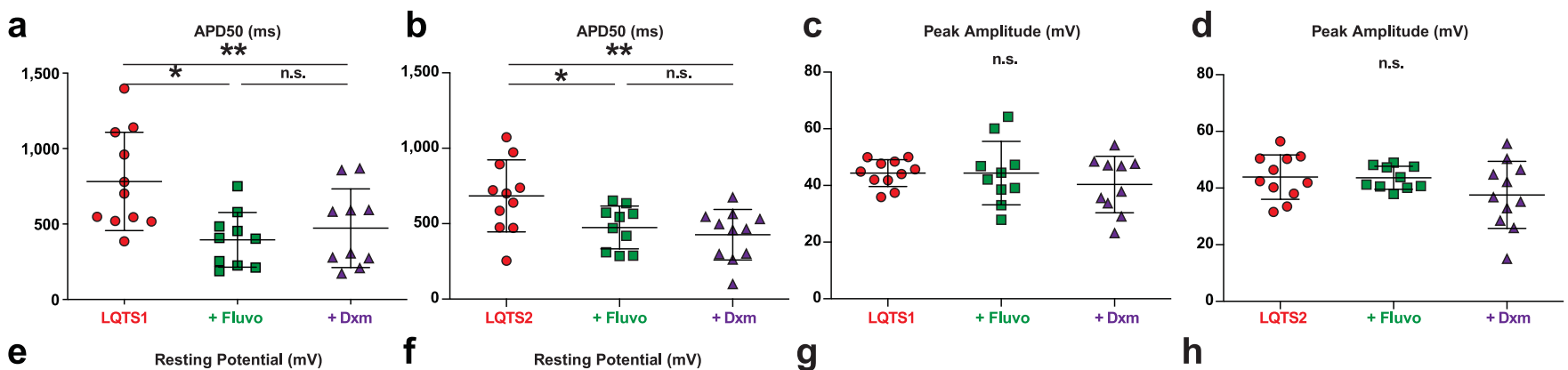

e
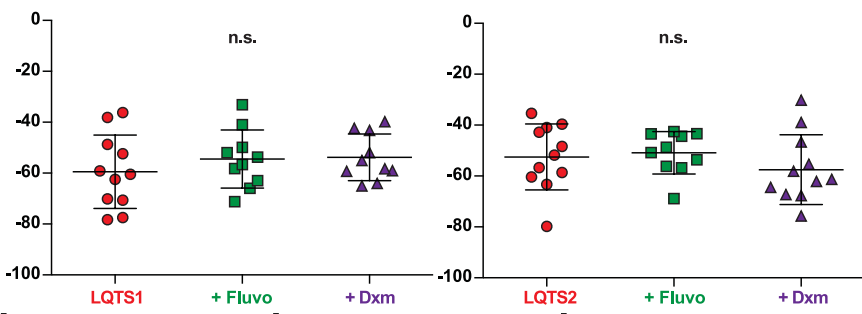

g

$I_{\mathrm{Kr}}$ tail $(\mathrm{pA} / \mathrm{pF})$

h

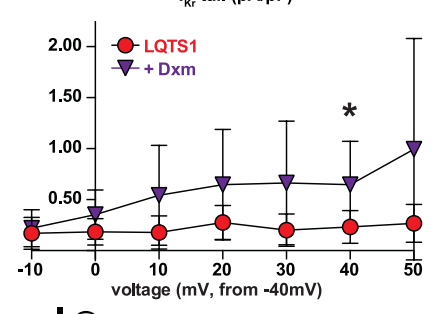

$I_{\mathrm{Kr}}$ (tail, $\mathrm{pA} / \mathrm{pF}$ )
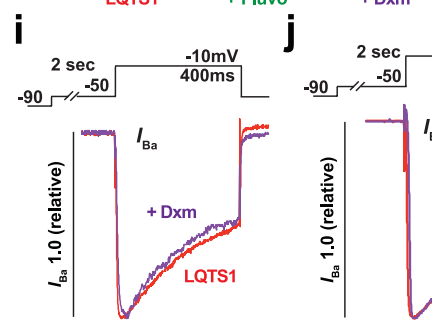

$\mathbf{k} \stackrel{\circ}{\circ}$
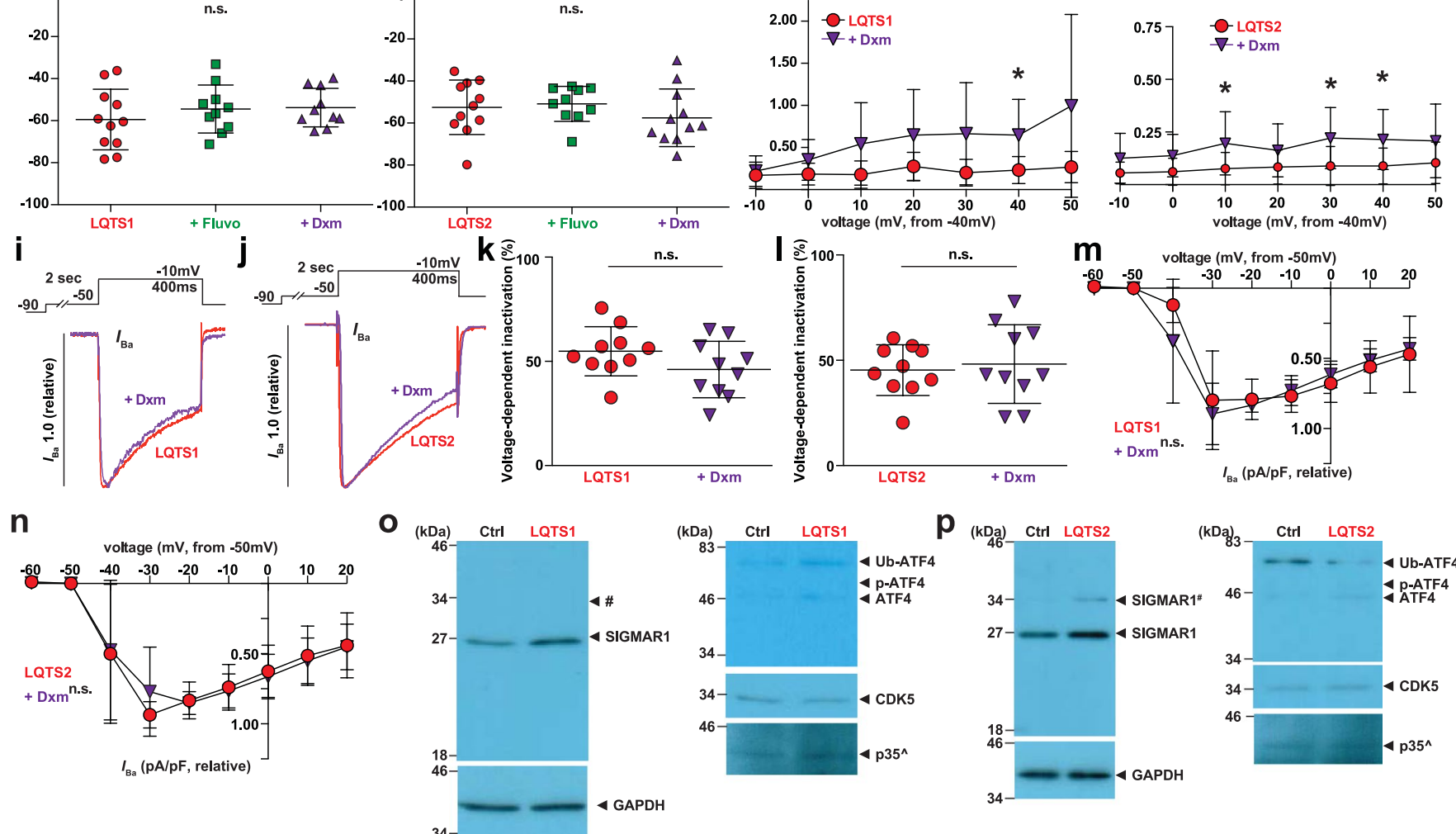

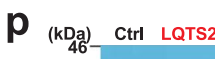
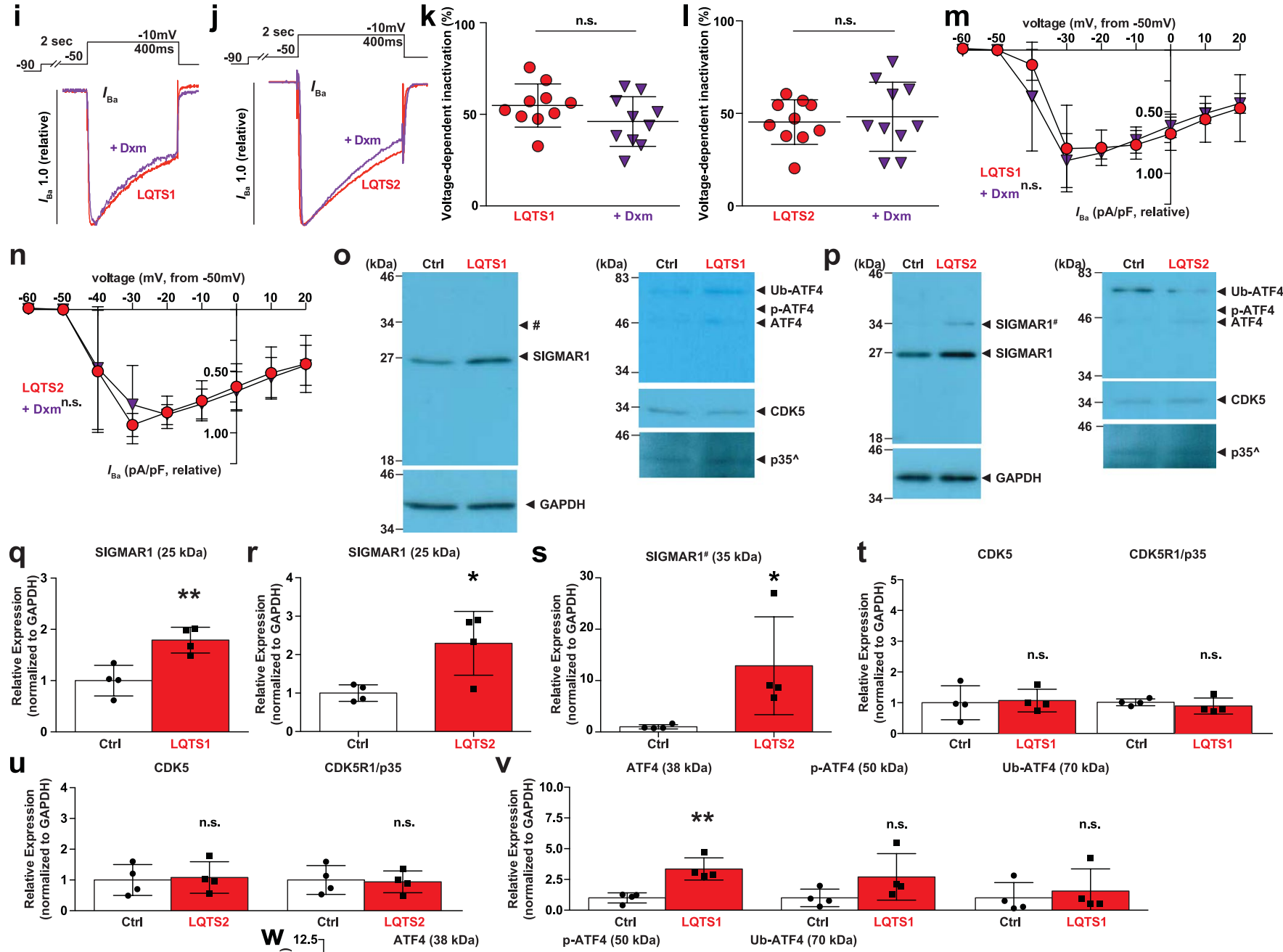

p-ATF4 (50 kDa)

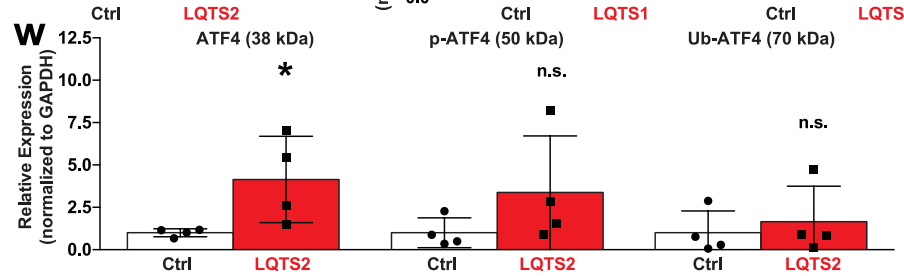

Extended Data Fig. 7 | See next page for caption. 
Extended Data Fig. 7 | The effect of dextromethorphan on the phenotypes in long QT syndrome type 1 and type 2. (a-f) Analysis of APD50 (50\% from peak, a, b), peak amplitude (c, d) and resting potential $(\mathbf{e}, \mathbf{f})$ in LQTS1 cardiomyocytes without treatment $(n=11)$ or treated with fluvoxamine (Fluvo, $n=10)$ or dextromethorphan (Dxm, $n=10)$ and in LQTS2 cardiomyocytes without treatment $(n=11)$ or treated with Fluvo $(n=10)$ or Dxm $(n=11)$. (g,h) $I_{\mathrm{Kr}}$ current (tail) was significantly increased by Dxm in LQTS1 cardiomyocytes at $40 \mathrm{mV}$ step ( $\left.\mathbf{g}, \mathrm{LQTS1}, n=10 ;+\mathrm{Dxm}, n=9\right)$ and in LQTS2 cardiomyocytes at 10, 30 and $40 \mathrm{mV}$ steps (h, LQTS2, $n=10 ;+D x m, n=9)$. (i,j) Representative traces of Ba ${ }^{2+}$ currents in LQTS1 (i) or LQTS2 cardiomyocytes $(\mathbf{j})$ without treatment or treated with Dxm. (k,I) Voltage-dependent calcium channel inactivation was not significantly changed by Dxm in LQTS1(k) or LQTS2 cardiomyocytes (I) compared to non-treated cells ( $n=10 /$ group). ( $\mathbf{m}, \mathbf{n})$ Current-voltage relationship of Ba ${ }^{2+}$ recordings in Dxmtreated $(n=10)$ and non-treated LQTS1 cardiomyocytes $(n=7)(\mathbf{m})$ and in Dxm-treated $(n=7)$ and non-treated LQTS2 cardiomyocytes $(n=8)(\mathbf{n})$. $(\mathbf{o}, \mathbf{p})$ Representative immunoblots of human SIGMAR1, ATF4, CDK5, CDK5R1/p35 and GAPDH protein using lysates from control (Ctrl) cardiomyocytes, LQTS1 cardiomyocytes (o) and LQTS2 cardiomyocytes (p). ^, p35 antibody used for Extended Data Fig. 1c was discontinued (Santa Cruz, sc-820). Therefore, another antibody (Cell Signaling Technology, C64B10) was used for this blotting series while its signal-to-noise ratio was not as high as sc-820. (q-s) Quantification of SIGMAR1 $25 \mathrm{kDa}$ protein band $(\mathbf{q}, \mathbf{r})$ and \#35 kDa protein band (s, possibly a dimer of a full-length SIGMAR1 with a SIGMAR1 splice variant ${ }^{59}$ ) in LQTS1 or LQTS2 cardiomyocytes compared to $\mathrm{Ctrl}(n=4 /$ group). ( $\mathbf{t}, \mathbf{u})$ Quantification of CDK5 (left) and CDK5R1/p35 protein expressions (right) in LQTS1 or LQTS2 cardiomyocytes compared to control cardiomyocytes ( $n=4 /$ group). ( $\mathbf{v}, \mathbf{w}$ ) Quantification of ATF4 38 kDa protein band (left, non-modified), $50 \mathrm{kDa}$ (center, phosphorylated) and $70 \mathrm{kDa}$ protein expression (right, ubiquitinated) in LQTS1 or LQTS2 cardiomyocytes compared to control cardiomyocytes ( $n=4 /$ group). All data are mean \pm s.d. The treatment of Dxm or Fluvo for the experiments was $5 \mu M, 2 \mathrm{hrs}$. One-way ANOVA with Tukey's multiple comparisons was used for a,b,c,d,e,f. Unpaired two-tailed Student's t-test was used for k,l, q,r,s,t,u,v,w between the groups and for g,h,m,n at each voltage step. ${ }^{\star} P<0.05,{ }^{\star \star} P<0.01$, n.s., not significant. The cardiomyocytes were from at least two independent differentiations. 
a

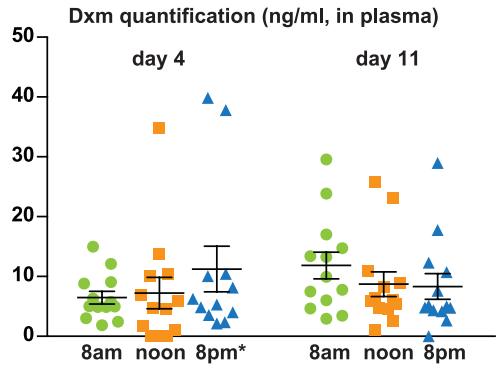

b

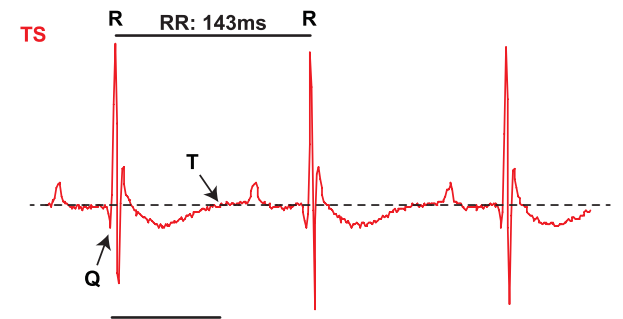

QT: $80 \mathrm{~ms}$

mouse $Q T c=Q T / S Q R T(R R / 100)=66.90 \mathrm{~ms} \quad \underline{50 \mathrm{~ms}}$

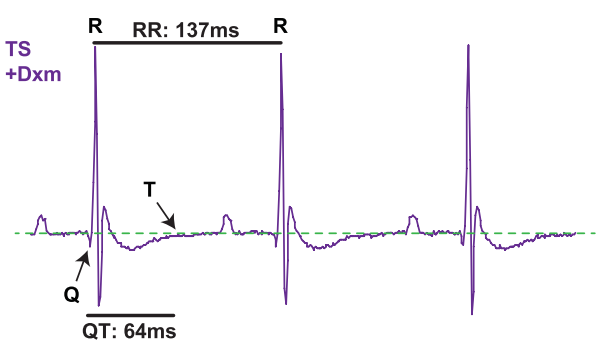

mouse $Q T c=Q T / S Q R T(R R / 100)=54.68 \mathrm{~ms}$

C

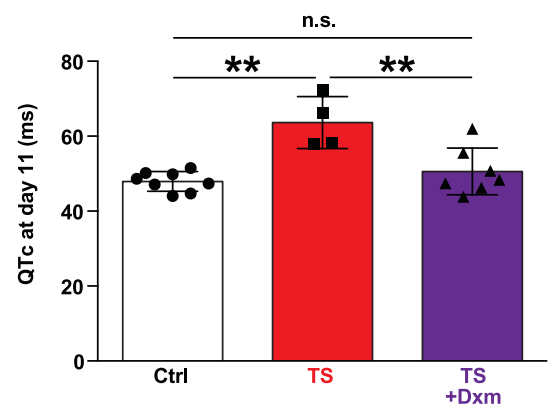

Extended Data Fig. 8 | The effect of dextromethorphan on Timothy syndrome mouse model. (a) Plasma dextromethorphan (Dxm) concentrations in control mice at day 4 and day 11 after continuous dosing of Dxm in drinking water $(n=13$, mean \pm s.e.m.). Dxm was provided in water since day 1 at 8 am. * 8 p.m. time point at day 4 had an outlier defined by statistics and the outlier was excluded from the final analysis. (b) Representative analysis procedures for ECG traces in Timothy syndrome (TS) mice without treatment or treated with Dxm at day 4 (also used in Fig. 5c,d). (c) QT intervals were significantly shortened by Dxm treatment in TS mice at day 11 ( $n=8$ for ctrl, $n=4$ for TS, $n=7$ for TS + Dxm, female, 12-week old). Three mouse heart samples from each group were used for the global proteomics analysis. All data of Extended Data Fig. 8 are mean \pm s.d. except Extended Data Fig. 8a. One-way ANOVA with Tukey's multiple comparisons were used for statistics. ${ }^{\star \star} P<0.01$, n.s., not significant. 

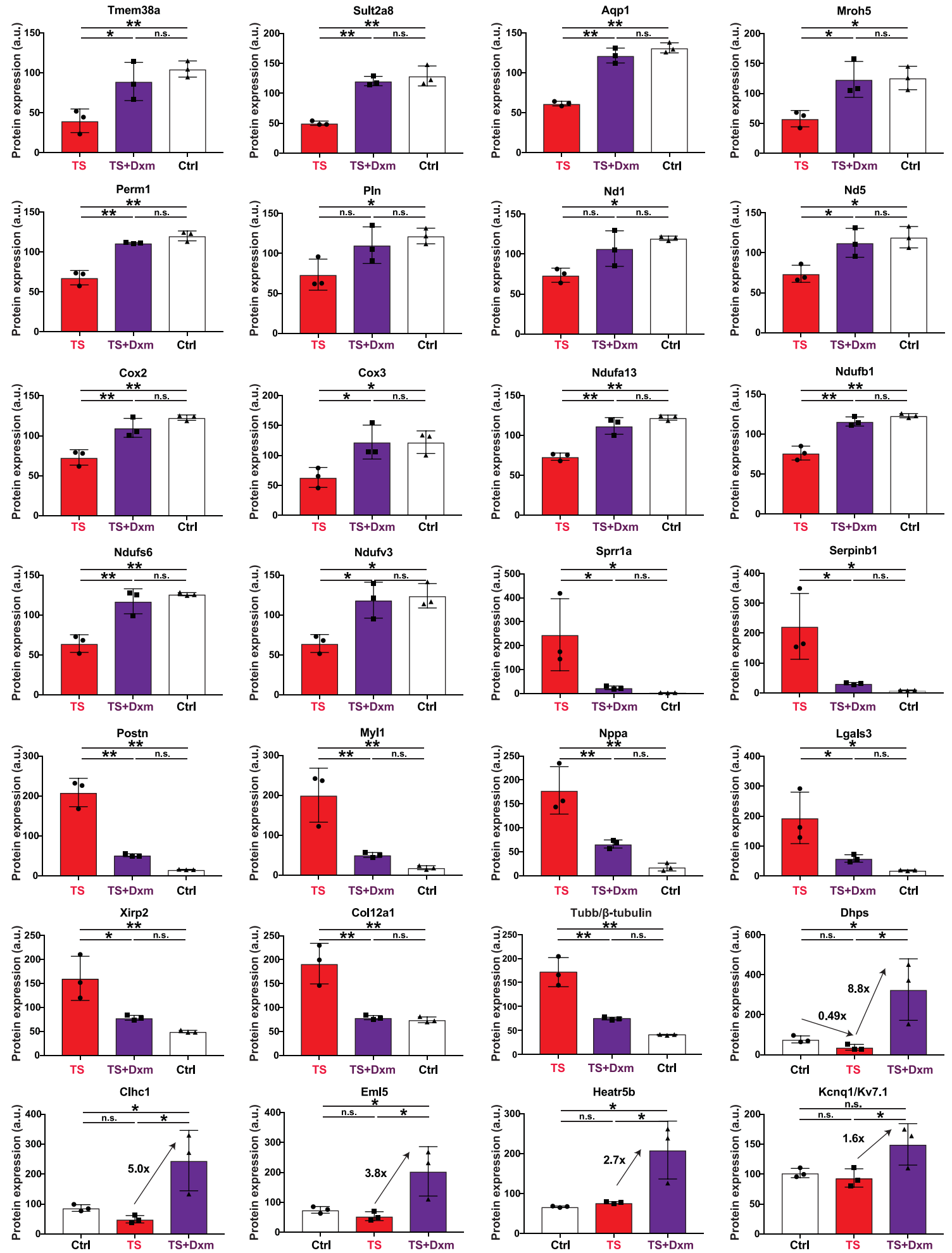

Extended Data Fig. 9 | The effect of dextromethorphan on the protein expression in mouse hearts. Altered protein expressions between control hearts (Ctrl), non-treated (TS) and Dxm-treated TS hearts (TS + Dxm) ( $n=3 /$ group). A comprehensive heat map and summary of proteomics results are shown in Fig. 5e. All data are mean \pm s.d. One-way ANOVA with Tukey's multiple comparisons was used. ${ }^{\star} P<0.05,{ }^{\star \star} P<0.01$, n.s., not significant. 

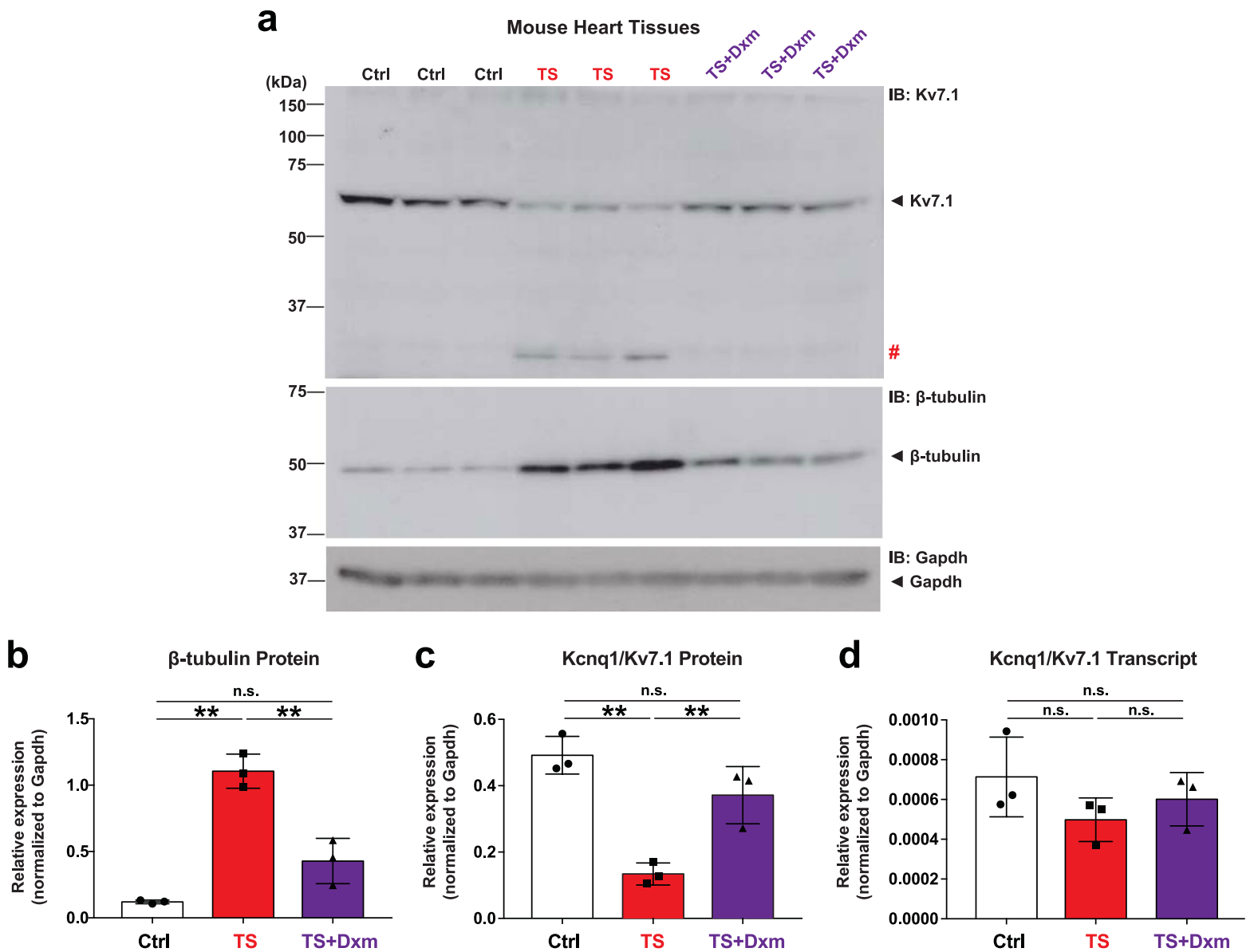

Extended Data Fig. 10 | The effect of dextromethorphan on $K_{v} 7.1$ protein expression in mouse hearts. (a) Representative immunoblots of $K_{v} 7.1, \beta$-tubulin and Gapdh protein using mouse heart lysates from TS (non-treated and Dxm-treated) mice and the control littermates (Ctrl) at day 11 (each, $n=3$, using the same samples with the global proteomics shown in Fig. 5e). \#, bands indicate putative degradation of $\mathrm{K}_{\mathrm{v}} 7.1$ in TS mouse hearts. (b-c) Quantification of $\beta$-tubulin (b) and Kcnq1/Kv 7.1 protein expression (c, normalized to Gapdh) in TS (non-treated and Dxm-treated) and Ctrl mouse hearts ( $n=3 / g r o u p$ ). Altered $\beta$-tubulin expression has been reported previously in animal model of myocardial infarction and Gapdh was recommended as a more reliable loading control for Western blot analyses ${ }^{60}$. (d) Kcnq1/K 7.1 mRNA expression (normalized to Gapdh) in TS (non-treated and Dxm-treated) and Ctrl mouse hearts ( $n=3$ /group). All data are mean \pm s.d. One-way ANOVA with Tukey's multiple comparisons was used. ${ }^{\star \star} P<0.01$, n.s., not significant. 


\section{nature portfolio}

Corresponding author(s): Masayuki Yazawa

Last updated by author(s): Dec 15, 2021

\section{Reporting Summary}

Nature Portfolio wishes to improve the reproducibility of the work that we publish. This form provides structure for consistency and transparency

in reporting. For further information on Nature Portfolio policies, see our Editorial Policies and the Editorial Policy Checklist.

\section{Statistics}

For all statistical analyses, confirm that the following items are present in the figure legend, table legend, main text, or Methods section.

n/a Confirmed

\The exact sample size $(n)$ for each experimental group/condition, given as a discrete number and unit of measurement

$\bigotimes$ A statement on whether measurements were taken from distinct samples or whether the same sample was measured repeatedly

The statistical test(s) used AND whether they are one- or two-sided

Only common tests should be described solely by name; describe more complex techniques in the Methods section.

$\bigotimes$ A description of all covariates tested

$\square$ A description of any assumptions or corrections, such as tests of normality and adjustment for multiple comparisons

$\triangle$ A full description of the statistical parameters including central tendency (e.g. means) or other basic estimates (e.g. regression coefficient)

$\triangle$ AND variation (e.g. standard deviation) or associated estimates of uncertainty (e.g. confidence intervals)

For null hypothesis testing, the test statistic (e.g. $F, t, r$ ) with confidence intervals, effect sizes, degrees of freedom and $P$ value noted

Give $P$ values as exact values whenever suitable.

Х $\square$ For Bayesian analysis, information on the choice of priors and Markov chain Monte Carlo settings

$\bigotimes \square$ For hierarchical and complex designs, identification of the appropriate level for tests and full reporting of outcomes

$\bigotimes \square$ Estimates of effect sizes (e.g. Cohen's d, Pearson's $r$ ), indicating how they were calculated

Our web collection on statistics for biologists contains articles on many of the points above.

\section{Software and code}

Policy information about availability of computer code

Data collection pClamp 10 (Molecular Devices), GLOMAX-Version 1.9.2 (Promega), StepOne Software Version 2.3 (Life Technologies), iox2 Version 2.8.0.19 (emka Technologies), Orbitrap Fusion, NIS Elements Version 4.51.01 (Nikon)

Data analysis Clampfit 10.4 (Axon Instruments), ecg-auto version 2.8.1.27 (emka Technologies), SWISS-MODEL (Biozentrum), CueMol2, Microsoft Excel for Mac 2011 (Microsoft), Prism Version 7.0d (GraphPad), NIS Elements Version 5.02.00 (Nikon), ImageJ-win64 (Fiji), PD2.2., Perseus statistical package (MaxQuant distribution), Ingenuity IPA, MATLAB R2009b (Mathworks).

For manuscripts utilizing custom algorithms or software that are central to the research but not yet described in published literature, software must be made available to editors and reviewers. We strongly encourage code deposition in a community repository (e.g. GitHub). See the Nature Portfolio guidelines for submitting code \& software for further information.

\section{Data}

Policy information about availability of data

All manuscripts must include a data availability statement. This statement should provide the following information, where applicable:

- Accession codes, unique identifiers, or web links for publicly available datasets

- A description of any restrictions on data availability

- For clinical datasets or third party data, please ensure that the statement adheres to our policy 


\section{Field-specific reporting}

Please select the one below that is the best fit for your research. If you are not sure, read the appropriate sections before making your selection.

$\bigotimes$ Life sciences $\quad \square$ Behavioural \& social sciences $\quad \square$ Ecological, evolutionary \& environmental sciences

For a reference copy of the document with all sections, see nature.com/documents/nr-reporting-summary-flat.pdf

\section{Life sciences study design}

All studies must disclose on these points even when the disclosure is negative.

Sample size Power analyses were conducted to minimize animal use following our IACUC protocol.

Data exclusions No samples were excluded from the analysis in this study, except one outlier in the analysis of dextromethorophan concentration in mouse plasma (Ext. Fig. 8a).

Replication At least two independent experiments (e.g. running two separately prepared experiments / using two independent differentiation-produced cells) were conducted to generate the data, except for the mass spectrometry analysis in Global Proteomics (Figure 5 and Ext. Figen

Randomization N/A.

Blinding The PLA assays, WGA staining analysis and ECG experiments were conducted by one individual and analyzed blindly by another individual or other individuals. The details have been noted in the method sections.

\section{Reporting for specific materials, systems and methods}

We require information from authors about some types of materials, experimental systems and methods used in many studies. Here, indicate whether each material, system or method listed is relevant to your study. If you are not sure if a list item applies to your research, read the appropriate section before selecting a response.

Materials \& experimental systems

$\mathrm{n} / \mathrm{a}$ Involved in the study

$\square$ 冈 Antibodies

$\square$ Eukaryotic cell lines

Х $\square$ Palaeontology and archaeology

$\square$ \ Animals and other organisms

$\bigotimes \square$ Human research participants

Х $\square$ Clinical data

$\bigotimes \square$ Dual use research of concern

\begin{tabular}{l} 
Methods \\
\hline n/a Involved in the study \\
$\square$ \\
$\square$ ChIP-seq \\
$\square$ Flow cytometry \\
$\square$
\end{tabular}

\section{Antibodies}

Antibodies used anti-CDK5 antibody-conjugated agarose beads (CDK5 (J-3) AC, Santa Cruz Biotechnology, \#:sc-6247 AC), anti-CDK5R1/p35 (Rabbit polyclonal Ab, \#:sc-820, Clone \# C-19, Santa Cruz), anti-CDK5 antibody (Mouse monoclonal Ab, \#:MABS50, Clone \# 1H3, Millipore), anti-beta-Tubulin antibody (Mouse mAb, \#:T5201, Clone \# TUB 2.1, Sigma Aldrich), HRP conjugate $\alpha$-mouse (Thermo Scientific, \#31430), HRP conjugate $\alpha$-rabbit (Thermo Scientific, \#31460), anti-KCNQ1/Kv7.1 antibody (Alomone lab, \# APC-022), anti-SIGMAR1 antibody (Santa Cruz, sc-137075), anti-SIGMAR1 (Abcam, ab53852), anti-HERG antibody (Santa Cruz, sc-377388), GAPDH antibody (Abcam, Catalog \# ab181602).

Validation All the antibodies used in the study are widely used and cited by other publications.

\section{Eukaryotic cell lines}

Policy information about cell lines

Cell line source(s)

Timothy syndrome iPSCs and normal control iPSCs were generated using a virus-free method from the skin fibroblasts and characterized as described previously (Yazawa M, et al. Nature. 2011 / Song L, et al. Stem Cells Transl Med. 2015 / Song L, et al. Stem Cell Reports. 2017). The isogenic control iPSCs were generated from Timothy syndrome iPSCs using gene editing technologies as described previously (Song L, et al. Stem Cell Reports. 2017). LQTS1 hiPSCs and LQTS2 hiPSCs were generated in Joseph Wu's lab at Stanford and shared with us. 
Commonly misidentified lines

(See ICLAC register)

\section{Animals and other organisms}

Policy information about studies involving animals; ARRIVE guidelines recommended for reporting animal research

Laboratory animals

Mus musculus, Female, Age: >11weeks, Strain: C57BI6/J (Envigo \# 044), Myh6-cre/Esr1 (The Jackson Laboratory, JAX\# 5657),

Conditional mouse model of Timothy Syndrome was generated by M.Y., targeting the Rosa26 locus with a G406R mutant in the calcium channel Cacna1c gene (Pasca, S. P. et al. Nature medicine. 2011). The detailed informations are specifically described in

"Mouse Electrocardiography" section of the Methods.

Wild animals
Field-collected samples
Ethics oversight

Study does not involve wild animals.

Study does not involve samples collected from the field.

Note that full information on the approval of the study protocol must also be provided in the manuscript.

Institutional Animal Care and Use Committee at Columbia University approved all animal experiments (\#AC-AAAU2453) 